\title{
Polycyclic aromatic hydrocarbons in the graphene era
}

\author{
Xiao-Ye Wang ${ }^{1,2^{*}}$, Xuelin Yao ${ }^{1} \&$ Klaus Müllen ${ }^{1 *}$ \\ ${ }^{1}$ Max Planck Institute for Polymer Research, Ackermannweg 10, 55128 Mainz, Germany; \\ ${ }^{2}$ State Key Laboratory of Elemento-Organic Chemistry, College of Chemistry, Nankai University, Tianjin 300071, China
}

Recieved February 25, 2019; accepted April 29, 2019; published online June 25, 2019

\begin{abstract}
Polycyclic aromatic hydrocarbons (PAHs) have been the subject of interdisciplinary research in the fields of chemistry, physics, materials science, and biology. Notably, PAHs have drawn increasing attention since the discovery of graphene, which has been regarded as the "wonder" material in the 21 st century. Different from semimetallic graphene, nanoscale graphenes, such as graphene nanoribbons and graphene quantum dots, exhibit finite band gaps owing to the quantum confinement, making them attractive semiconductors for next-generation electronic applications. Researches based on PAHs and graphenes have expanded rapidly over the past decade, thereby posing a challenge in conducting a comprehensive review. This study aims to interconnect the fields of PAHs and graphenes, which have mainly been discussed separately. In particular, by selecting representative examples, we explain how these two domains can stimulate each other. We hope that this integrated approach can offer new opportunities and further promote synergistic developments in these fields.
\end{abstract}

polycyclic aromatic hydrocarbon, graphene, graphene nanoribbon, nanographene, graphene quantum dot, carbon materials

Citation: Wang XY, Yao X, Müllen K. Polycyclic aromatic hydrocarbons in the graphene era. Sci China Chem, 2019, 62: 1099-1144, https://doi.org/10.1007/ s11426-019-9491-2

\section{Introduction}

Carbon allotropes and carbon-based materials have recently attracted such attention that one even describes the current era as the carbon age [1,2]. These materials can appear as discrete particles or bulk materials, and their structures can be crystalline or amorphous. One can easily understand why "diamonds are a girl's best friend" but should also consider that soot, a structurally ill-defined product of incomplete combustion of carbonaceous matter, is of enormous technical importance, such as in mechanical reinforcement of plastics. Graphite is a carbon allotrope wherein hexagon networks of $\mathrm{sp}^{2}$-hybridized carbons are stacked on the top of each other. Graphene is a single sheet out of this multilayered material and can also be regarded as a giant two-dimensional (2D)

*Corresponding authors (email: xiaoye.wang@nankai.edu.cn; muellen@mpip-mainz.mpg.de) conjugated polymer [3-10]. Although graphene has been predicted to be thermodynamically unstable in early theoretical studies [11-13], the groundbreaking work by Geim and Novoselov et al. [14], after peeling off separate graphene layers from graphite, has changed the world of materials science.

Graphene nanoribbons (GNRs) are quasi-one-dimensional (1D) graphene cutouts, typically with widths in the range of $1-100 \mathrm{~nm}$ and length/width ratios $>10$ (Figure 1) [6]. GNRs can also be considered as an intermediate between graphene and polycyclic aromatic hydrocarbons (PAHs). Comparing the electronic features of the quasi-1D GNRs with those of 2D graphene reveals interesting insights. Reviewing the outstanding properties of graphene clarifies the crucial role of such GNRs in electronics. In particular, the ultrahigh charge-carrier mobility appears favorable when graphene is used as an active component of field-effect transistors (FETs) [15]. However, this advantage is hampered by the vanishing 
electronic band gap, and consequently, a current cannot be switched off between source and drain electrodes. An opening of the band gap and thus a significant on-off ratio could be achieved by a geometric confinement as shown in GNRs $[6,16]$. The fabrication of GNRs is possible through so-called top-down methods, as represented by the "cutting" of graphene and the "unzipping" of carbon nanotubes [6]. The obtained GNRs with different widths are promising to use in FET devices with high on-off ratios $[17,18]$ (up to $10^{6}$ for a $2 \mathrm{~nm}$-wide GNR) and high charge-carrier mobilities [19] (up to $1,500 \mathrm{~cm}^{2} \mathrm{~V}^{-1} \mathrm{~s}^{-1}$ ). Nevertheless, the top-down methods cannot accurately control the GNR structures such as the edges and widths at the atomic level. This precision would, however, be necessary for future electronics $[15,20]$. Therefore, the inverse method, namely, bottom-up chemical syntheses from molecular precursors, has emerged as an indispensable way to reproducibly synthesize structurally well-defined GNRs with atomic precision. The role of GNRs as a new family of semiconductors is further enhanced by two attractive design opportunities in the bottom-up protocol: (1) by varying the aspect ratio of the ribbons, the band gaps can be finely tuned to control charge transport properties $[21,22]$; (2) their electronic band structures also depend on the nature of peripheries such as armchair, zigzag, and cove edges (for definitions, see Section 3.1). Moreover, zigzag structures, when synthesized to perfection, provide entry into new areas such as spintronics and enable fabrication of topological insulators [23,24]. Further reducing the dimensionality of graphene leads to quasi-zero-dimensional (0D) graphene quantum dots (GQDs), which are graphene cutouts with lateral sizes ranging from 1 to $100 \mathrm{~nm}$ [6]. GQDs are semiconductors with non-zero band gaps, displaying size-dependent optical and electronic properties $[25,26]$. When the lateral size of PAHs is larger than $1 \mathrm{~nm}$, they are indeed a type of atomically precise GQDs and are sometimes also referred to as nanographene molecules $[6,27]$. These nanoscale graphenes, including the quasi-1D GNRs and quasi-0D GQDs as well as nanographene molecules, are collectively termed nanographenes [6].

A common theme in the theoretical analyses of extended $\pi$-systems is the extrapolation from finite to infinite structures. In this context, a powerful principle for understanding linear conjugated polymers is building up a homologous series of monodisperse oligomers and the expected convergence of their properties toward those of the corresponding polymers. This "oligomer approach" is proven valuable because oligomers have acquired importance not only as model compounds but also as materials in their own right $[28,29]$. Thus, a good case could be presented by evaluating the performance of oligomers as active components of devices [30-32]. The same concept could also be applied to PAHs and graphenes to explore the interconnection between these two classes of materials.
Indeed, PAHs have played a key role in the evolution of modern organic chemistry. Their structural description is tightly connected to names of pioneers such as Robinson and Clar $[33,34]$. The tremendous flow of graphene research, on the other hand, may now suggest several key questions: Do PAHs, with defined molecular structures, contribute to the understanding of graphenes? Can new PAHs, their synthesis, processing, and application, even offer advantages over graphenes? In this context, linear extension of the PAHs (e.g., hexa-peri-hexabenzocoronene ( $p$-HBC) in Figure 1) leads to 1D oligomer-like counterparts and eventually GNRs, which can be regarded as an analog of 1D conjugated polymers. As 2D polymers have emerged as an established concept in polymer science [35], 2D "oligomers" can be introduced here to represent the link between the "monomeric" PAHs (e.g., $p$-HBC) and graphene. By adding "shell" structures to the existing PAH core, a sequence of larger 2D PAHs can be obtained, eventually extending to infinite graphene [36]. However, extensions of PAHs in 1D and 2D result in completely different electronic consequences [37]. Specifically, 1D extension to GNRs converges at a defined band gap, whereas 2D extension to infinite graphene causes vanishing of the band gap. Therefore, the 2D extrapolation from PAHs to graphene is less straightforward because the fundamental difference in the electronic state diagrams of molecular PAHs and 2D graphene prohibits direct correlation of their electronic properties. Nevertheless, PAHs can effectively serve as model compounds of GNRs to understand how the structural features (e.g., size, edge configuration, and heteroatom incorporation) influence the electronic properties of GNRs. Notably, PAHs also play different roles in the synthesis of GNRs and graphene. In the bottom-up synthesis of GNRs, PAHs are often used as molecular precursors, which are polymerized and then cyclodehydrogenated to provide tailor-made GNRs $[6,10,16]$. However, the bottom-up synthesis of graphene is preferably furnished by chemical vapor deposition (CVD) from gaseous precursors (e.g., $\mathrm{CH}_{4}$ and $\mathrm{C}_{2} \mathrm{H}_{5} \mathrm{OH}$ ) [38,39] or epitaxial growth on $\mathrm{SiC}$ wafers [40,41], although PAHs sometimes, but very rarely, are used as solid carbon sources for CVD [42]. Overall, PAHs exhibit greater significance in the context of GNRs than in the filed of graphene regarding the role of PAHs as molecular models and synthetic precursors.

The value of PAHs is proven, in particular, when deriving reliable structure-property relations for precise structures. Major examples, which are discussed in the following, concern the role of (1) different edge topologies, (2) peripheral functionalizations, (3) defined "defects" such as nonsix-membered rings and holes, or (4) site-selective heteroatom incorporation. Additional value for elucidating graphenic structures comes from crystallographic analysis of PAHs, which provides quantitative information on features such as bond-length alternation and packing modes in the 


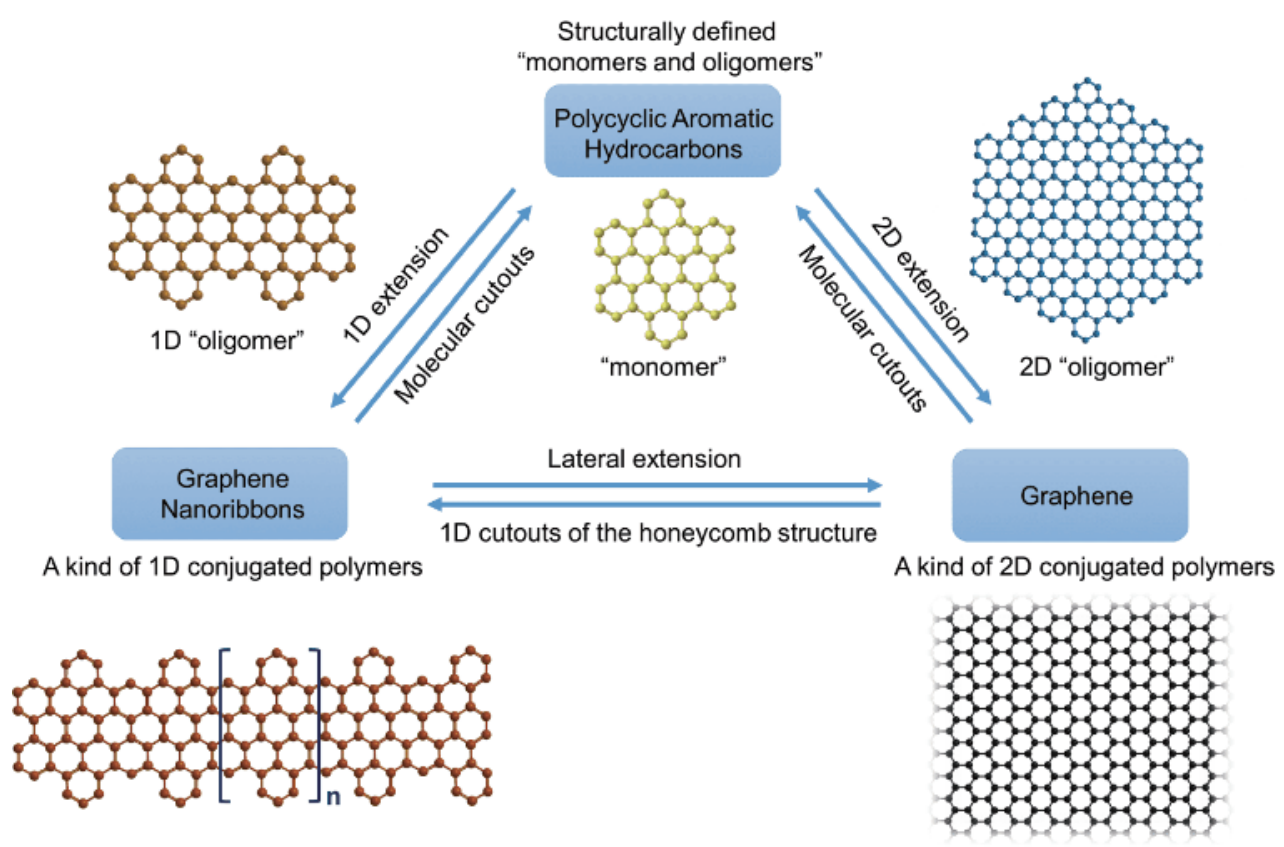

Figure 1 Golden triangle of polycyclic aromatic hydrocarbons (PAHs), graphene nanoribbons, and graphene (color online).

solid state [43].

For a long time, PAHs have attracted attention from different fields of sciences, and one may argue that PAHs have been located at important crossroads of chemical research. A few cases should be mentioned: (1) elementary molecular orbital theory has been developed with PAHs as ideal test cases whose properties such as resonance energies and atom localization energies could be readily varied by changing the modes of benzene-ring fusion [44-46]; (2) organic dye-stuff chemistry has often established chromophore structures by combining PAH cores with auxochromic groups [47-49]; (3) astrophysics has been searching for PAHs as carbon-containing materials in interstellar space [50,51]; (4) pentacene, a case with a linear mode of benzene-ring fusion, is regarded as a prototype of organic semiconductors [52]; (5) PAHs with 4 and 5 benzene cores such as benzo $[a]$ pyrene are metabolized by initial enzymatic epoxidation and are cancerogenic [53]; (6) PAHs have opened an entry into chirality by means of their helical derivatives such as helicenes [54-57]. Despite the value of PAHs, they have been relegated to the backseat, becoming mainly an issue of toxicological analyses due to the increasing role of life sciences and synthetic method developments in organic chemistry. However, in the last two decades, PAH chemistry has seen an enormous revival (Figure 2) from synthetic and functional perspectives. Readers may decide whether this condition is closely related to the appearance of graphene materials. Nonetheless, other developments have further stimulated PAH research. Among these are the ingenious invention of scanning probe methods that have enabled the visualization of PAHs in real space or even their use as single-molecule electronic devices [58-60].
These developments have peaked in a unique combination of surface science, synthetic chemistry, and scanning tunneling microscopy (STM), i.e., the synthesis of conjugated polymers, PAHs and GNRs after immobilization of precursors on metal surfaces, and direct monitoring of the process by STM [61-63]. Other research topics that have stimulated new investigations of PAHs relate to their broad applications, such as in organic electronic and optoelectronic devices, as well as in bioimaging and sensing $[30,64,65]$. These advances together with the rise of graphene have generated renewed interest in PAHs over the past decades [66-71]. This review, however, mainly focuses on PAHs in the "graphene context" as well as the mutual interactions of the PAH chemistry and the research interest in GNRs and graphene.

Thus far, we have studied molecularly defined, "individual" carbon-based chain or disc structures and the relevant structure-property relations. This approach is too narrow in view of another characteristic feature of materials science, that is, the need to create a defined macroscopic state of matter before putting a material to work. This "processing" often targets the solid state or thin films on surfaces. In FET devices, for example, charge-carrier transport between source and drain electrodes could, in principle, employ a single chain or sheet structure, but more often relies on ensembles of molecules [72]. For conjugated polymers, major prerequisites for high charge-carrier mobilities are a high degree of supramolecular order and, in most cases, a preferable edge-on arrangement of the polymers on the surface [73]. A similar motif, stacking into columnar order and edge-on deposition, would be needed for PAH discs as well [74]. GNRs that are hoped to combine the advantage of 

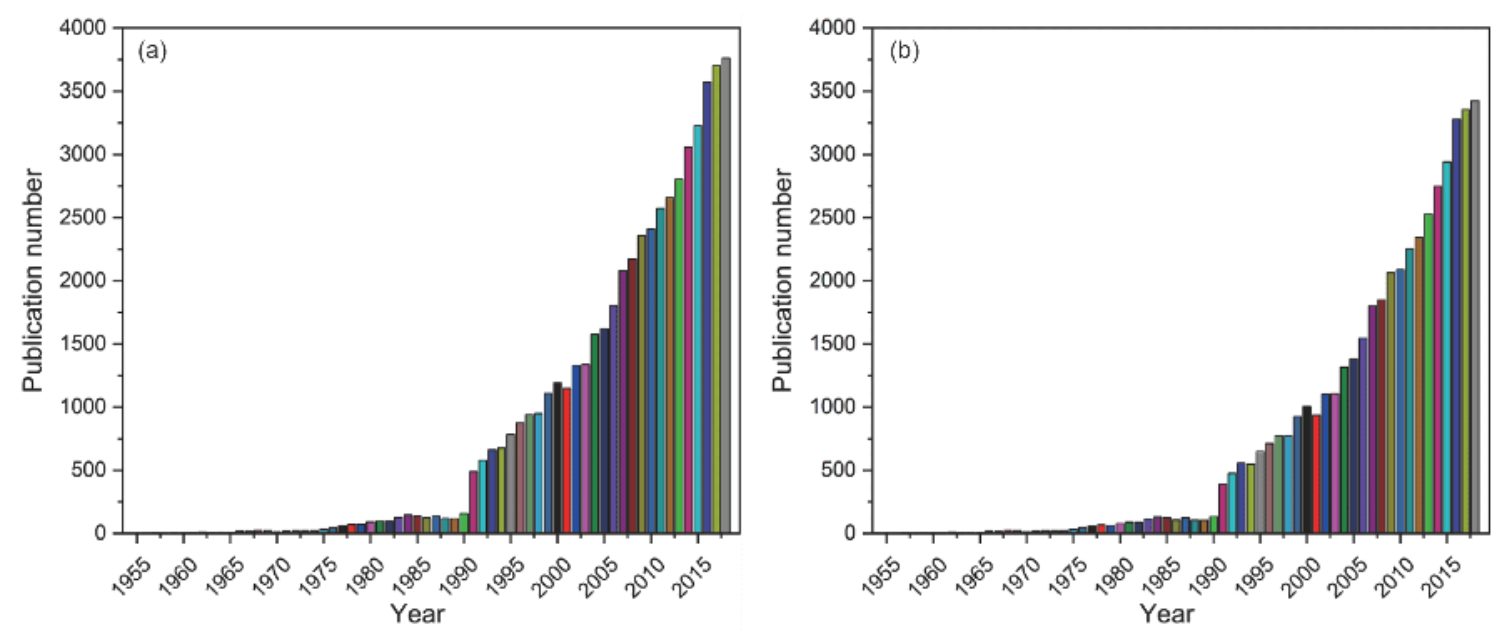

Figure 2 (a) Distribution of publications by year using "polycyclic aromatic hydrocarbons" as keyword for searching on the Web of Science; (b) Same distribution diagram but excluding publications on toxicology studies (color online).

high mobilities from graphene with opened band gaps, and thus on-off behavior of FETs, would tend to adopt a face-on packing on the surface $[17,18]$. In this case, however, the overall mobility also depends on charge transport between the separate stripes; again, this is a supramolecular issue. Another critical aspect in the use of both chain and disc structures is the solubility in organic solvents. Reduced solubility with increasing molecular weight inhibits further growth during synthesis. On the other hand, solubility is a prerequisite for film formation from solution. This deposition is a complex process which, among others, implies aggregation, phase formation, and nucleation prior to the formation of thin layers. The solubility of oligomeric and polymeric chains can be enhanced by alkyl substituents. While such substituents are in danger of hampering conjugation due to steric hindrance, they can also support supramolecular order [75]. The same is true for PAHs where the nanophase separation between soft alkyl chains and hard aromatic cores leads to the existence of discotic mesophases $[74,76]$. A similar concept is unrealistic for the large graphene flakes, and alternative protocols are needed to process such 2D materials.

Conjugated oligomers and polymers, PAHs and graphenes have been extensively reviewed from different angles comprising fundamental and application-oriented aspects [3$6,10,16,32,52,67,74,77-81]$. The reader is advised to consult the insightful texts cited in this article (and also those that may have been overlooked). How can the present article, being far from comprehensive, still contribute to the field? The present piece is intended to describe the promising role of PAHs in light of graphene research and sketch a few trends by which both fields have stimulated each other. The story of their linear (quasi-1D) counterparts can thereby serve as helpful references. What the materials sciences of both conjugated polymers and graphenes have in common is their strongly interdisciplinary nature with emphasis on physical properties and device behavior. However, materials must be first fabricated, thereby requiring the competence of synthetic chemistry. This review leans toward chemistry-driven achievements from molecular and supramolecular points of view. The reason is that robust technologies are derived not only from physical properties established in a complex system but also from the "reliability" of the material. This condition implies not only ease of production and costs but also structural perfection of the products. In the following, we first introduce the synthetic strategies toward PAHs (Section 2), and then discuss the recent advances in the PAH chemistry and mutual interactions between the fields of PAHs and graphenes (Section 3). Finally, we summarize and propose future opportunities (Section 4).

\section{Synthetic strategies for PAHs}

\subsection{Two-stage synthesis: precursor formation followed by a "stitching" step}

The various concepts of PAH synthesis often comprise two key stages: formation of precursors and further ring closure ("stitching" or "graphitization") to generate the fused ring system (Figure 3). For $p$-HBC as an example, hexaphenylbenzene (HPB) 1 can be obtained by (1) metal-catalyzed cross-coupling reactions of 1,4-diiodo-2,3,5,6tetraphenylbenzene 2 with phenylboronic acid 3, (2) cobaltmediated $[2+2+2]$ cyclotrimerization of diphenylacetylene $\mathbf{4}$, or (3) Diels-Alder reaction between diphenylacetylene 4 and tetraphenylcyclopentadienone $\mathbf{5}$; then, the HPB precursor is subjected to a Scholl reaction in the presence of chemical oxidants, yielding the planarized $p$-HBC 6. A number of PAHs have been synthesized according to this two-stage protocol [79]. Although various methods are available to 
construct the precursors from small $\pi$-moieties, the important factors are the ring-closure reactions for the "stitching" step to furnish the final PAHs. These "stitching" methods are discussed in the following.

The Scholl reaction has undoubtedly played an indispensable role in the final stage of PAH synthesis [82]. This reaction, which is described as proceeding through either a radical cation pathway or an arenium cation pathway (Figure 4), is highly efficient in forming multiple carbon-carbon bonds in one step and powerful in synthesizing large PAHs. A number of PAHs have been synthesized by the Scholl reaction (see previous reviews) [67,74,80,81], such as $p$ HBC derivatives and hexagonal PAH 16 comprising 222 carbons with a diameter of approximately $3 \mathrm{~nm}$ by Müllen $e t$ al. [83] (Figure 5(a)). The broad scope of the Scholl reaction has not only been demonstrated in the formation of planar PAHs, but also in the syntheses of non-planar structures in spite of the existing strain. For example, Scott and Itami et al. [84] described a palladium-catalyzed direct $\mathrm{C}-\mathrm{H}$ arylation of corannulene 17 to provide precursors 18 and 19 , followed by the Scholl reaction, yielding the grossly warped nanographene molecules $\mathbf{2 0}$ with five- and seven-membered rings (Figure 5(b)). Despite the significant steric demand, the reaction works efficiently to obtain highly curved PAHs in good yields $(50 \%-62 \%)$. However, in some cases, the Scholl reaction involves drawbacks such as undesired chlorination and unexpected structural rearrangements [83,85-87].

Another ring-closure method that involves a radical mechanism is flash vacuum pyrolysis (FVP), which is normally conducted under high temperatures [88,89]. FVP is extremely useful in preparing bowl-shaped (geodesic) PAHs and fullerenes. For instance, by using the FVP method, Scott et al. [90] demonstrated the chemical synthesis of fullerene $\mathbf{2 2}$ from a rationally designed precursor 21 (Figure 6(a)), and

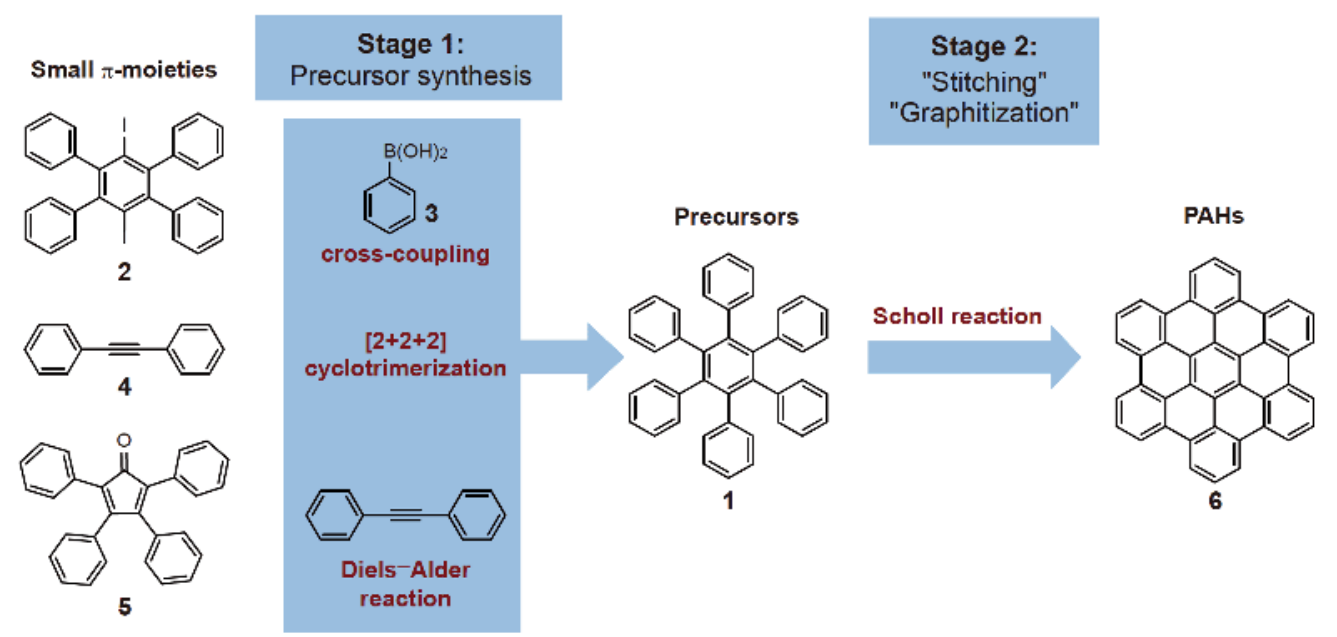

Figure 3 Illustration of two-stage synthetic protocol of PAHs with $p$-HBC (6) as an example (color online).

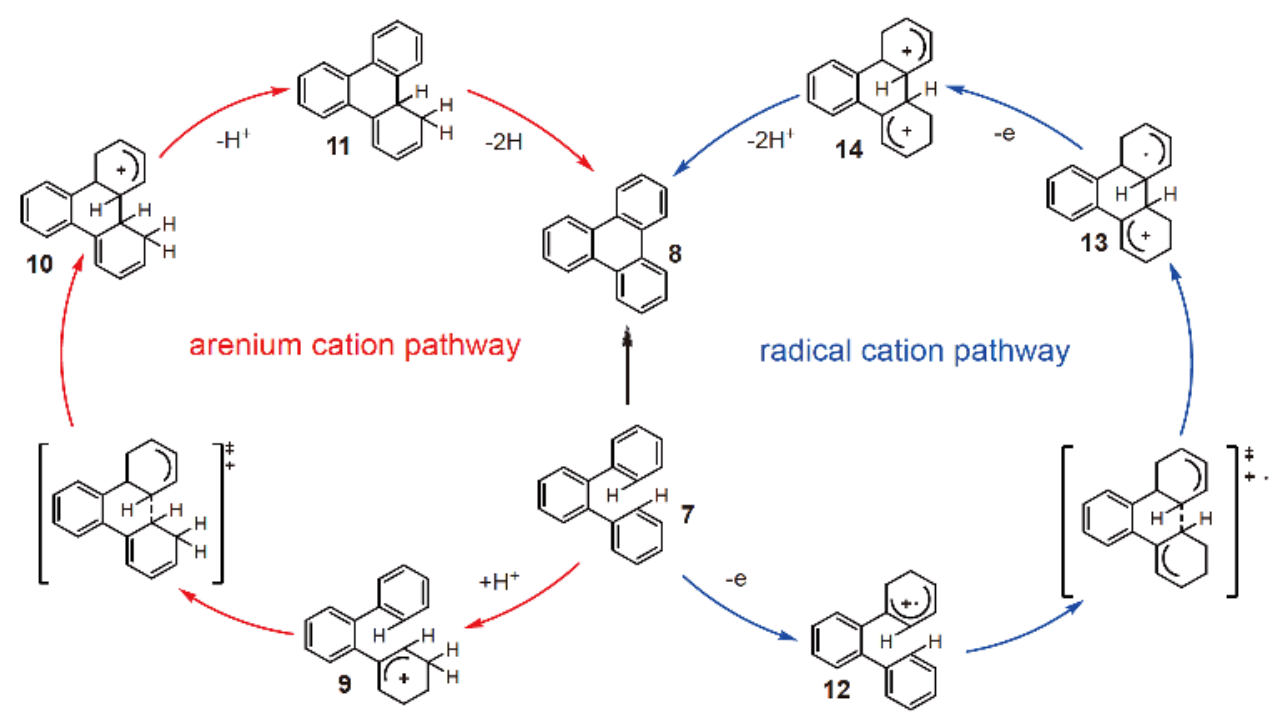

Figure 4 Two possible mechanisms of Scholl-type cyclodehydrogenation reaction (color online). 


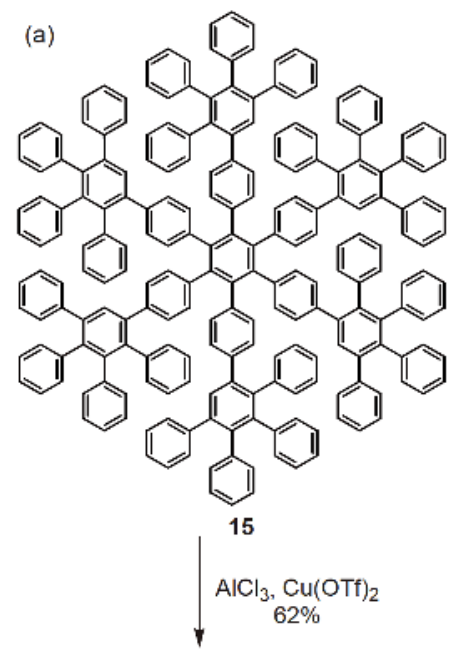

(b)
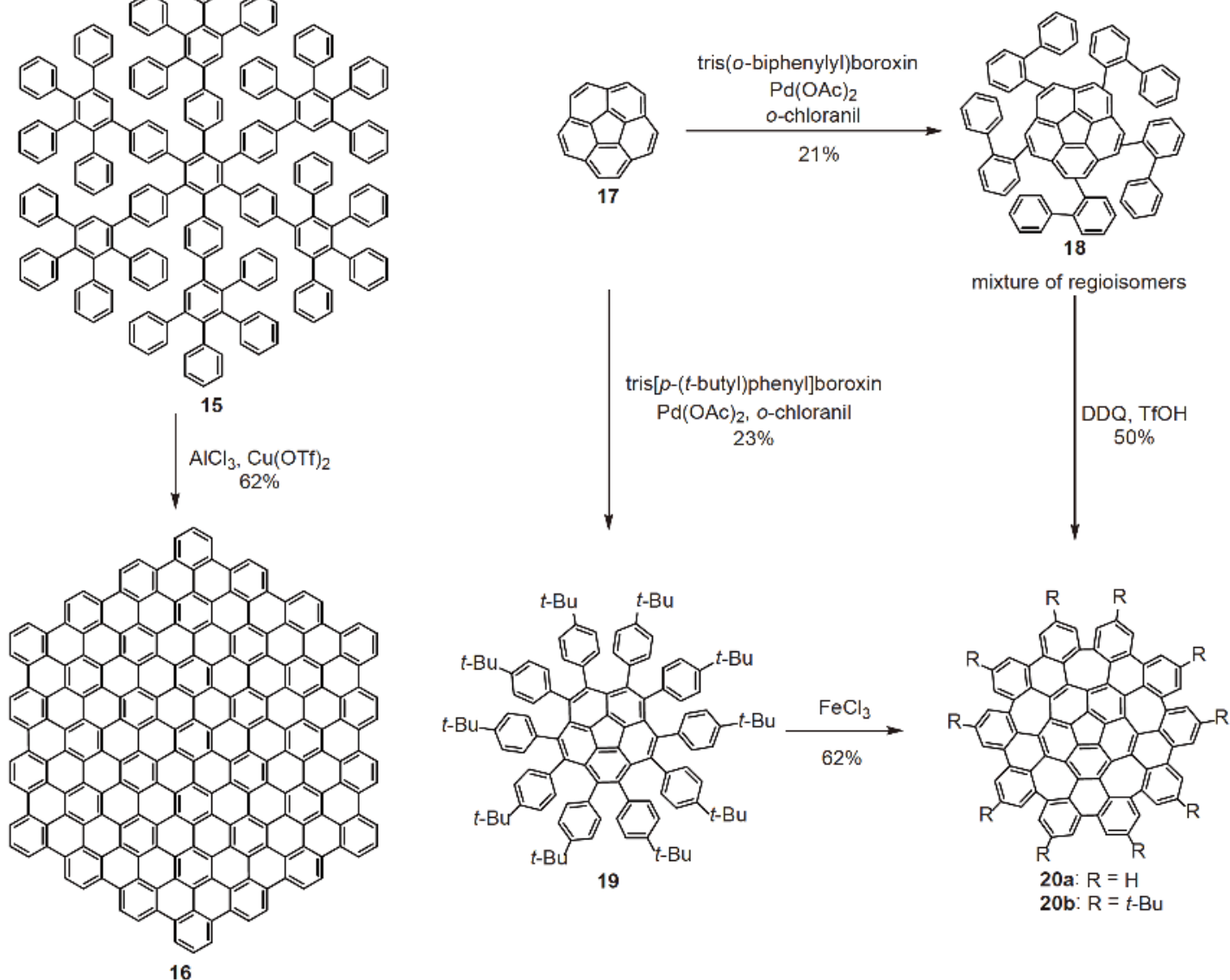

Figure 5 Application of Scholl reaction to synthesize (a) PAH 16 with $222 \mathrm{sp}^{2}$-carbons and (b) non-planar PAH 20. Cu(OTf) $)_{2}$ : copper(II) trifluoromethanesulfonate; DDQ: 2,3-dichloro-5,6-dicyano-1,4-benzoquinone; TfOH: trifluoromethanesulfonic acid (color online).
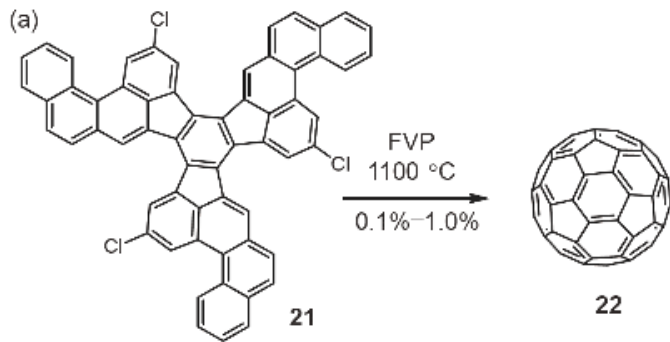

(b)
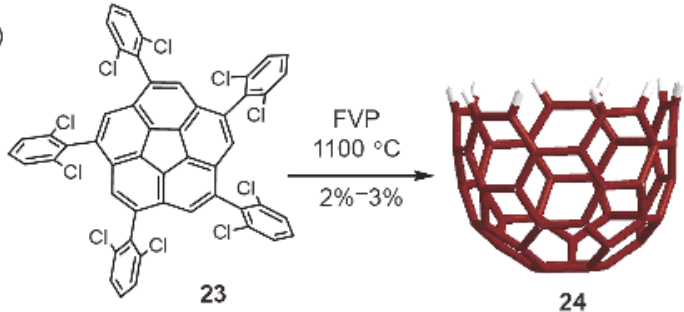

Figure 6 Flash vacuum pyrolysis (FVP) for preparing (a) fullerene 22 and (b) bowl-shaped PAH 24 (color online).

of a deeply bowl-shaped PAH $24\left(\mathrm{C}_{50} \mathrm{H}_{10}\right)$ from a phenylsubstituted corannulene derivative 23 (Figure 6(b)) [91]. The yield of such FVP reactions is generally low. Introduction of halogens (e.g., chlorine) in the precursors, as described above, facilitates the generation of radical intermediate and thus improves the yield to a certain extent.

Photochemical cyclization of stilbene and related compounds provides an alternative way to perform aryl-aryl ring closures and has proven valuable in the synthesis of PAHs. Under UV irradiation, the stilbene moiety 25a undergoes an electrocyclic rearrangement forming dihydrophenanthrene 26a, which further aromatizes into phenanthrene 27 in the presence of oxidants such as iodine or oxygen (Figure 7(a)) [92-94]. A representative example is the synthesis of hexacata-hexabenzocoronenes 29 (c-HBCs, Figure 7(b)) by Nuckolls et al. [95] through photocyclization of stilbene-type precursors 28. The photocyclization can also proceed through an elimination mechanism involving no oxidants (Figure 7(a)). In this case, a functional group X (e.g., halogens) has to be present in the stilbene moiety $(\mathbf{2 5 b})$ at the ortho-position of one phenyl ring; upon a photo-induced electrocyclic reaction, the loss of $\mathrm{HX}$ from a $4 \mathrm{a}-\mathrm{H}, 4 \mathrm{~b}-\mathrm{X}-$ dihydrophenanthrene-type intermediate $\mathbf{2 6 b}$ drives the reaction to the aromatized product. This type of photocyclization reactions employing halogens in the precursors, 

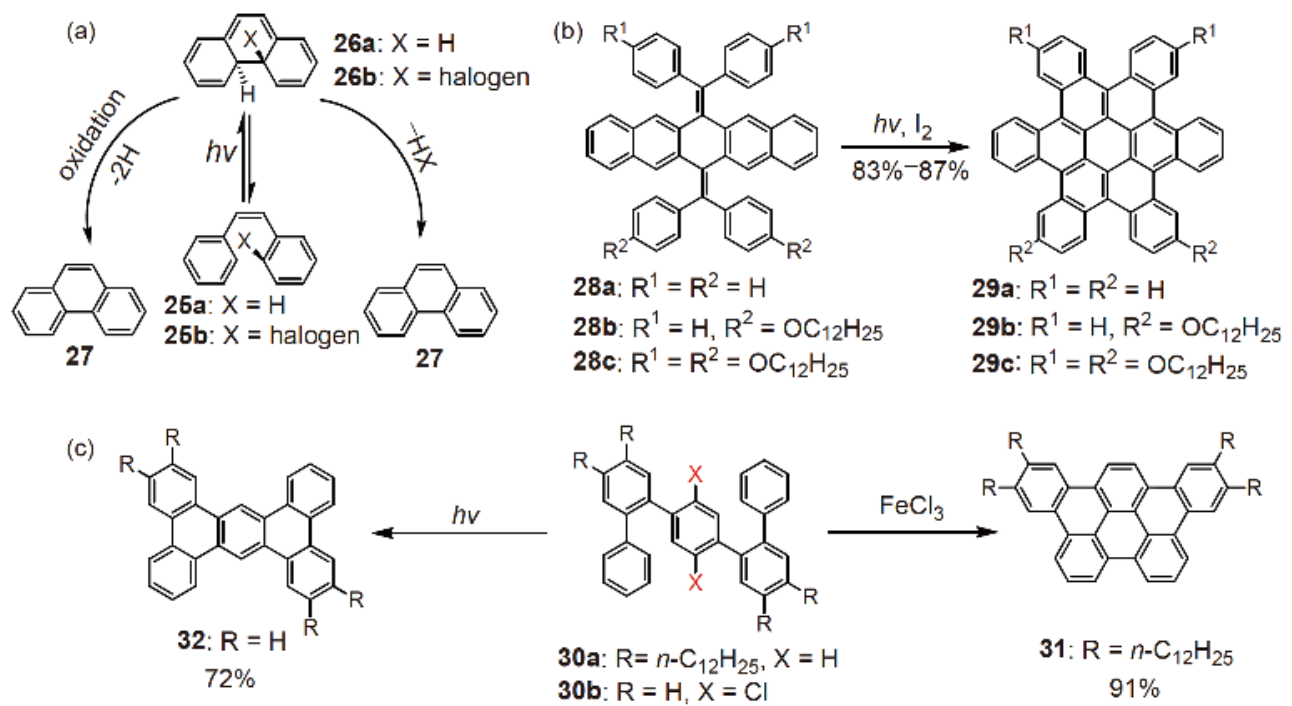

Figure 7 Photochemical cyclization for synthesis of PAHs. (a) Two pathways toward phenanthrene via oxidation or HX elimination; (b) synthesis of hexacata-hexabenzocoronenes; (c) photocyclodehydrohalogenation vs. Scholl reaction (color online).

which is often described as a photocyclodehydrohalogenation, is highly regioselective, providing PAHs, which are otherwise difficult to synthesize. For instance, starting from the similar oligophenylenes (Figure 7(c)), Müllen et al. [96] demonstrated that precursor 30a was cyclized efficiently under the Scholl reaction to provide PAH 31 in 91\% yield, whereas Morin et al. [97] showed that precursor 30b, on which two chlorine atoms were introduced, led to PAH 32 in $72 \%$ yield through photocyclodehydrohalogenation.

Indeed, the installation of halogens in the precursors at the ring-closing positions facilitates the cyclization in many ways. This approach is also important in modern transitionmetal-catalyzed cross-coupling reactions for PAH synthesis. In particular, the palladium-catalyzed intramolecular Heck reaction (also referred to as intramolecular $\mathrm{C}-\mathrm{H}$ arylation) between $\mathrm{Ar}-\mathrm{X}(\mathrm{X}=$ halogens $)$ and $\mathrm{Ar}-\mathrm{H}$ has proven not only valuable in the synthesis of planar PAHs but also essential to the construction of strained bowl-shaped PAHs, such as pentaindenocorannulene 34 synthesized by Scott et al. [98] (Figure 8(a)). There are some cases where transition-metal catalysts are not needed. Specifically, $\mathrm{Al}_{2} \mathrm{O}_{3}$-mediated intramolecular aryl-aryl coupling between $\mathrm{Ar}-\mathrm{F}$ and $\mathrm{Ar}-\mathrm{H}$ has been demonstrated by Amsharov et al. [99] through HF elimination, forming strained compounds such as PAH 36 (Figure 8(b)).

Developments of modern techniques have also allowed the cyclodehydrogenation on metal surfaces under ultrahigh vacuum (UHV). The metal surfaces function as a catalyst in the ring-closure reaction and the UHV keeps a clean environment that prevents potential side reactions. Through this surface-assisted method, the resulting structures can be clearly visualized in-situ by state-of-the-art high-resolution STM and non-contact atomic force microscopy (nc-AFM) (a)
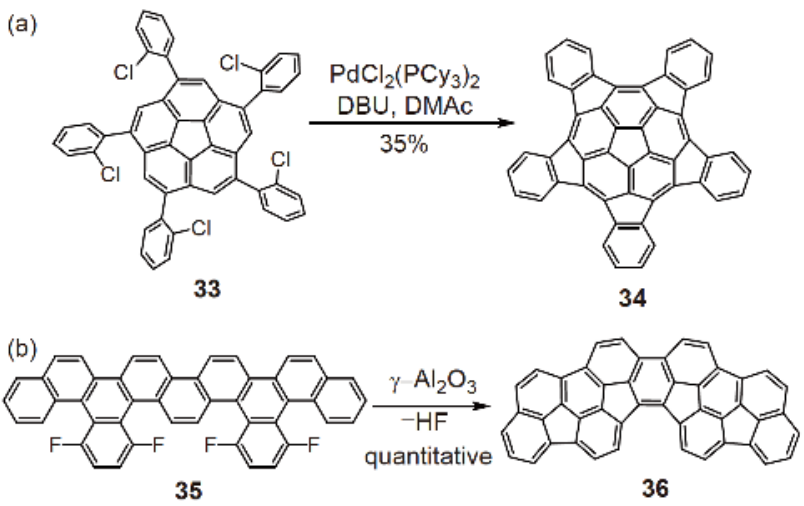

Figure 8 Synthesis of (a) PAH 34 through palladium-catalyzed intramolecular Heck reaction and (b) $\mathrm{PAH} 36$ via $\mathrm{Al}_{2} \mathrm{O}_{3}$-mediated $\mathrm{C}-\mathrm{H}$ arylation. $\mathrm{PdCl}_{2}\left(\mathrm{PCy}_{3}\right)_{2}$ : dichlorobis(tricyclohexylphosphine)palladium(II); DBU: 1,8-diazabicyclo[5.4.0]undec-7-ene; DMAc: dimethylacetamide.

[100]. For example, Fasel et al. [101] demonstrated the synthesis of a triangular nanographene molecule, tribenzo[ $a$, $g, m]$ coronene 38, on $\mathrm{Cu}(111)$ surfaces under UHV by cyclodehydrogenation of a cyclic polyphenylene precursor, namely, cyclohexa-o-p-o-p-o-p-phenylene 37, and characterized the product directly by STM (Figure 9). The onsurface synthesis and characterization techniques can also be applied to GNR synthesis, which is described in Section 3.

\section{$2.2 \pi$-Extension with extra carbons and rings}

Apart from the two-stage synthetic protocol, as described above, PAHs can also be synthesized via $\pi$-extension of existing PAHs. One representative example is the Diels-Alder reaction at the bay regions of PAHs, which have a dienelike character and thus can react with various dienophiles 
(a)

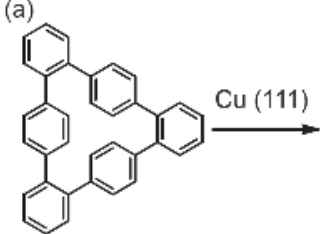

37

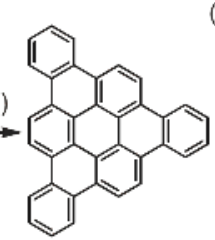

38

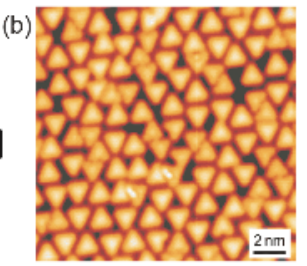

Figure 9 (a) Surface-assisted synthesis of PAH 38 via cyclodehydrogenation on $\mathrm{Cu}(111)$ surface; (b) high-resolution STM image of PAH 38 on $\mathrm{Cu}(111)$ surface. Reprinted with permission from Ref. [101], copyright (2010) Macmillan Publishers Ltd. (color online).

such as arynes, alkenes, alkynes, and quinones to produce extended PAH structures [102]. For example, Clar et al. [103] reported consecutive Diels-Alder reactions of perylene 39 with maleic anhydride to eventually obtain the pristine coronene 43 (Figure 10(a)). Scott et al. [104] demonstrated that bisanthene $\mathbf{4 4}$ readily reacted with dienophile nitroethylene to afford ovalene $\mathbf{4 5}$ (Figure 10(b)), whereas a smaller homologue, perylene $\mathbf{3 9}$, reacted with nitroethylene much more slowly even under harsher conditions and only single-side fused product $\mathbf{4 6}$ could be observed (Figure 10 (c)).

Other approaches to extend the bay region of PAHs relate to the simplest case: how to make phenanthrene from a biphenyl. The solutions include established methods, such as McMurry coupling of 2,2'-diformylbiphenyl [105] and ringclosing olefin metathesis [106]. To demonstrate the synthesis of PAHs by intramolecular olefin metathesis, King et al. [107] synthesized dibenzo[ $[a, j]$ anthracene $\mathbf{4 8}$ and dibenzo[ $a$, $h]$ anthracene $\mathbf{5 0}$ from two isomeric tetravinyl-substituted terphenyl precursors in excellent yields $(87 \%-95 \%$, Figure 11(a)). Moreover, starting from 2,2'-diformylbiphenyl precursors, Wang et al. [108] developed a one-pot two-step synthesis of phenanthrene derivatives through rhodium(II)catalyzed cyclization of $N$-tosylhydrazone-based inter- mediates (Figure 11(b)). This method was also employed to furnish a series of larger PAHs, such as chrysene $\mathbf{5 2}$ and picene 54 [108]. Another widely used method is alkyne cyclization promoted by electrophilic reagents (Figure 11(c)) [109]. For example, Larock et al. [110] reported ICl-induced intramolecular cyclization of 2-(arylethynyl)biphenyls (e.g., 55), providing PAHs with iodo groups, which can be used for further functionalizations. Swager et al. [111] developed alkyne benzannulations induced by trifluoroacetic acid (TFA) to afford a series of PAHs such as molecule 58. An electron-rich aryl group attached to the acetylene moiety is essential to this reaction. Such alkyne cyclizations can also be triggered by metal catalysts, e.g., $\mathrm{Ru}, \mathrm{Pt}$, and $\mathrm{Au}$ [112]. An exemplary case has been reported by Scott et al. [113] through a short synthesis of coronene via four-fold Ru-catalyzed alkyne benzannulations. The relatively low yield of this reaction $(15 \%-20 \%)$ has been significantly improved to $86 \%$ by Liu et al. [114] through optimization of the $\mathrm{Ru}$ catalyst and reaction conditions.

A different strategy to readily extend PAH structures is cyclotrimerization. For example, Peña et al. [115] performed palladium-catalyzed cyclotrimerization of an aryne intermediate $\mathbf{6 1}$ for efficient preparation of star-shaped PAH $\mathbf{6 2}$ (Figure 12(a)). With such a method, the size of PAHs grows quickly. However, synthesis via cyclotrimerization of arynes often requires multi-step preparation of the aryne precursors. In this regard, cyclotrimerization of $o$-dibromoarenes through Yamamoto coupling can serve as a complementary approach. Indeed, such a method has been employed by Bunz et al. [116] to synthesize large starphenes 64 and $\mathbf{6 6}$ from linear tetracene and pentacene derivatives 63 and 65 (Figure 12(b)), respectively.

Transition-metal catalysts apparently provide a number of new opportunities for PAH syntheses. While the importance of aryl-aryl coupling to form carbon-carbon single bonds in synthesizing the precursors is unquestionable, the annulative
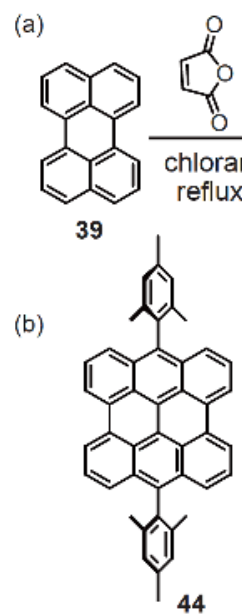

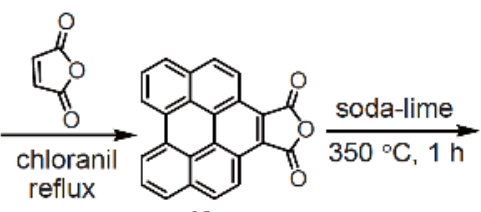

40
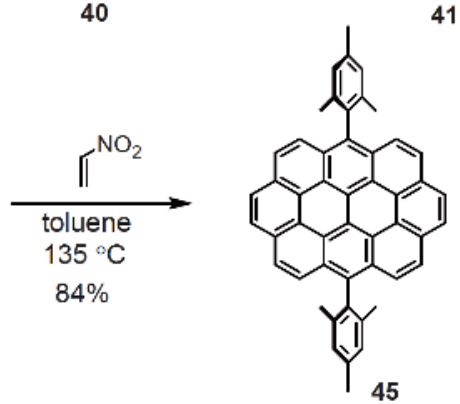

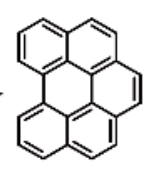

41

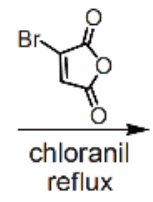

(c)

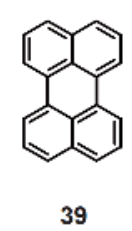

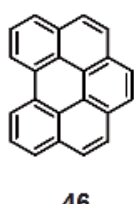

46

Figure 10 (a) Synthesis of pristine coronene 43 via $\pi$-extension of perylene at bay regions by Diels-Alder reactions; (b) Diels-Alder reaction between bisanthene 44 and nitroethylene; (c) Diels-Alder reaction between perylene 39 and nitroethylene. 
(a)

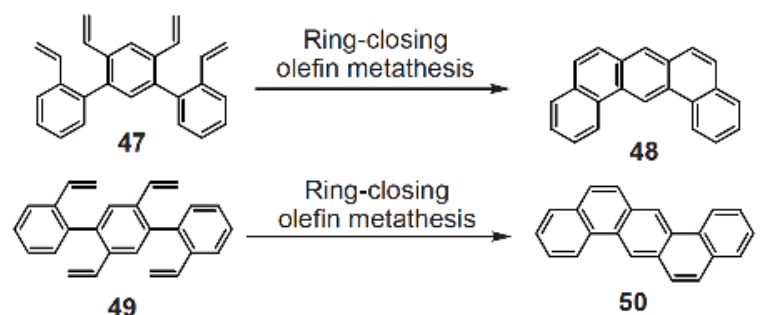

(b) $\mathrm{R}^{1}=0$
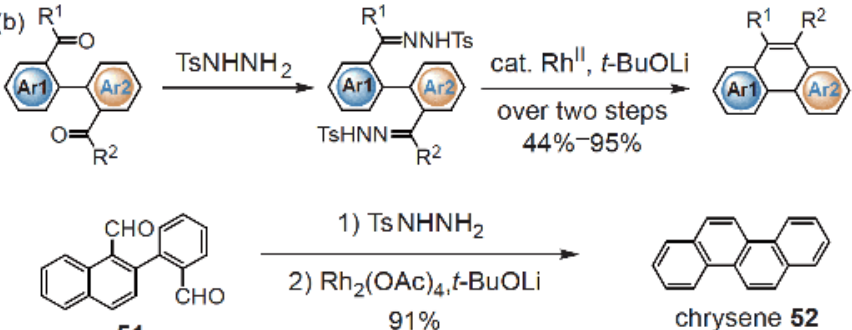

51

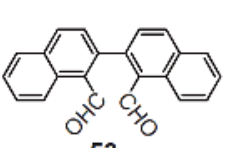

53

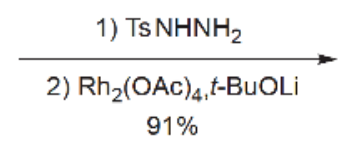

1) $\mathrm{TsNHNH}_{2}$ toluene, $60^{\circ} \mathrm{C}$

2) $\mathrm{Rh}_{2}(\mathrm{OAC})_{4}, t$-BuOLi $86 \%$
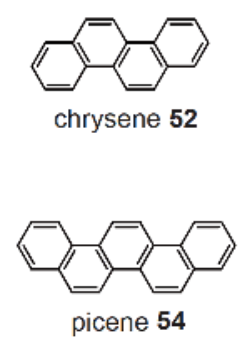

(c)<smiles>Cc1ccccc1-c1ccccc1C#Cc1ccccc1</smiles><smiles>Ic1c(-c2ccccc2)c2ccccc2c2ccccc12</smiles>

56

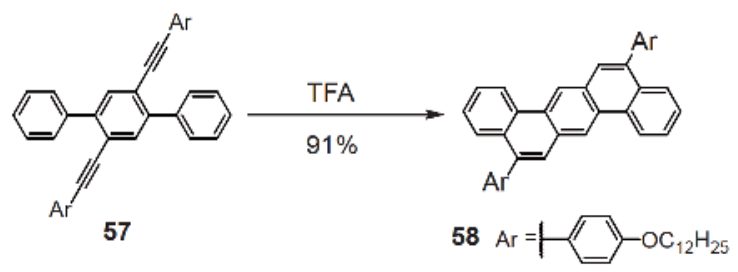<smiles>C#CC(C#C)=c1c2ccccc2c(=C(C#C)C#C)c2ccccc12</smiles>

59 condition 1: $\begin{array}{ll}\mathrm{Ru}(\mathrm{Cymene}) \mathrm{Cl}_{2} \mathrm{PPh}_{3} \\ \mathrm{C}_{2} \mathrm{H}_{2} \mathrm{Cl}_{4}, \text { reflux }\end{array}$

condition 2: $\left.\left[\mathrm{Ru}\left(\mathrm{CH}_{3} \mathrm{CN}\right)_{2}\left(\mathrm{PPh}_{3}\right) \mathrm{TP}\right] \mathrm{PF}_{6}\right] \quad 86 \%$ $\mathrm{C}_{2} \mathrm{H}_{2} \mathrm{Cl}_{4}, 80^{\circ} \mathrm{C}$

Figure $11 \pi$-Extension toward different PAHs through (a) ring-closing olefin metathesis, (b) rhodium(II)-catalyzed cyclization of $N$-tosylhydrazone-based intermediates, and (c) alkyne cyclization. TsNHNH 2 : p-toluenesulfonyl hydrazide; $\mathrm{Rh}_{2}(\mathrm{OAc})_{4}$ : dirhodium tetraacetate; TFA: trifluoroacetic acid (color online).

(a) (b)

63

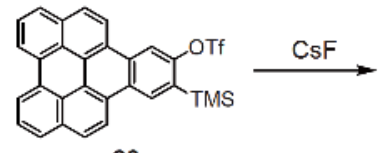

60

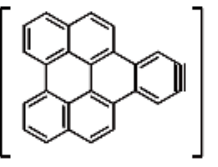

61

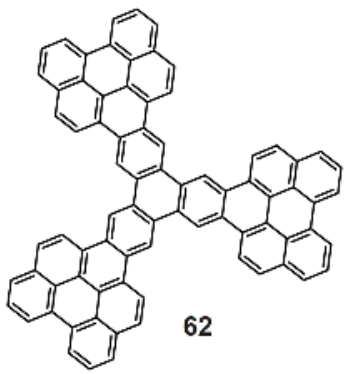

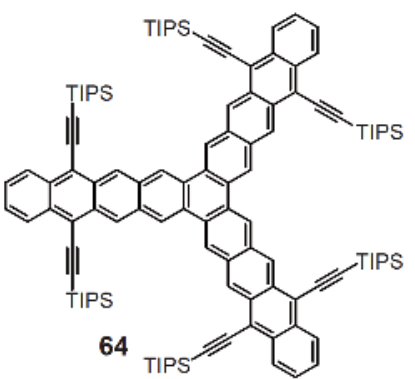
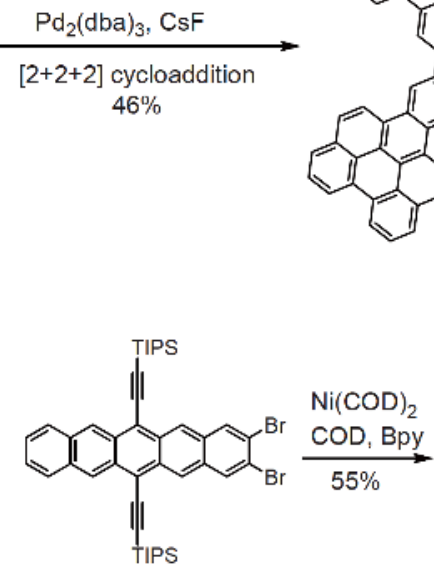

65

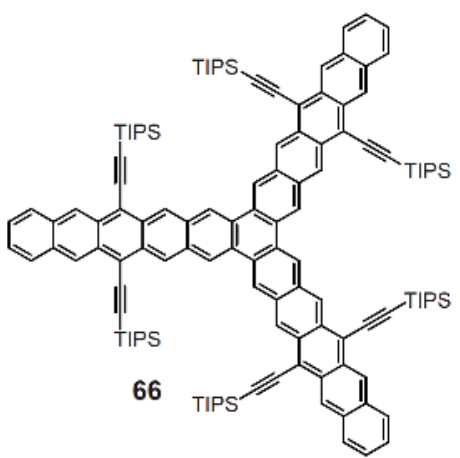

Figure 12 Cyclotrimerization toward extended PAHs. (a) Synthesis of star-shaped PAH 62 through palladium-catalyzed cyclotrimerization; (b) synthesis of starphenes 64 and 66 via Yamamoto coupling. $\mathrm{Pd}_{2}(\mathrm{dba})_{3}$ : tris(dibenzylideneacetone)dipalladium(0); COD: 1,5-cyclooctadiene; Bpy: 2,2'-bipyridine.

$\pi$-extension, in which more than one single aryl-aryl bond is formed, is more intriguing with regard to direct fusion of aromatics. The aforementioned cyclotrimerization belongs to such a category. In 2009, Shimizu et al. [117] introduced an annulation method through a Pd-catalyzed double crosscoupling reaction of 9,9-dimethyl-9-stannafluorenes 67 and 1,2-dibromobenzene 68, furnishing triphenylene 69 in $90 \%$ yield (Figure 13). Tetrabromoarenes (e.g., 70) were also employed for double-side annulations to synthesize larger PAHs such as 71. In a similar manner, Li, Negri and Wang et al. [118] synthesized a $\pi$-extended naphthalene diimide 73, which is promising as an air-stable n-type semiconductor. 


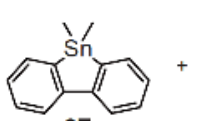

67

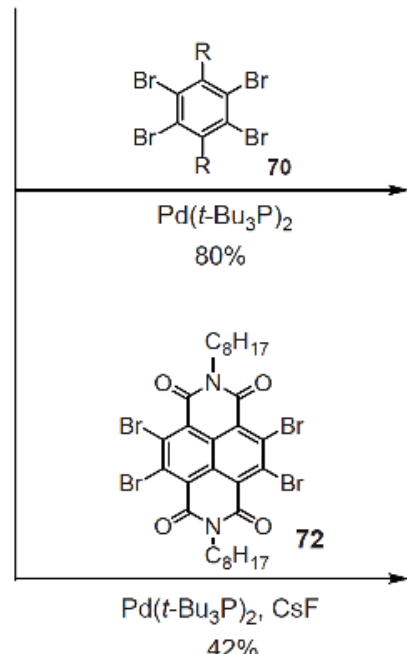

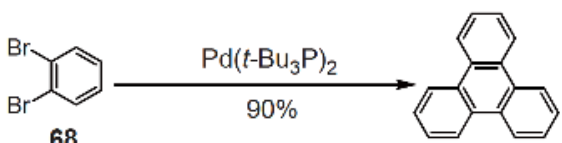

69

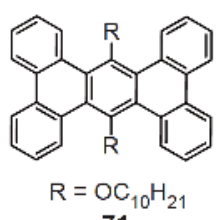

71

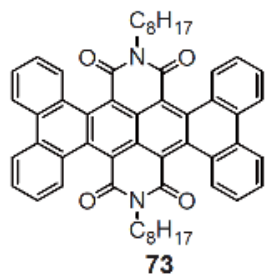

Figure 13 Synthesis of PAHs 69, 71 and 73 through annulative $\pi$-extension by palladium-catalyzed double cross-couplings. $\operatorname{Pd}\left(t-\mathrm{Bu}_{3} \mathrm{P}\right)_{2}$ : bis(tri-tert-butylphosphine)palladium(0).

$\pi$-Annulation by employing aryl halides and alkynes in the presence of palladium catalysts provides cyclopentannelated arenes (Figure 14). Garcia-Garibay et al. [119,120] reported such reactions based on brominated anthracenes and terminal alkynes. Müllen et al. [121] further explored the cyclopentannulation reaction using di-substituted alkynes. The reaction proceeds through the insertion of the alkyne $\mathbf{7 5}$ into an arylpalladium species $\mathbf{7 7}$, followed by intramolecular electrophilic attack of the resulting alkenylpalladium $\mathbf{7 8}$ on anthracene to form a palladacycle intermediate 79 (a type of $\mathrm{C}-\mathrm{H}$ activation step). Subsequent reductive elimination affords the corresponding cyclopentannelated product 76. This reaction can be performed on various PAHs, such as anthracene, pyrene, and perylene, to generate corresponding singly and doubly cyclopentannelated derivatives 80-84.

Itami et al. [122] developed a double $\mathrm{C}-\mathrm{H}$ arylation to achieve an annulative $\pi$-extension at the K-regions (olefinic region) of PAHs (Figure 15). For example, phenanthrene 86 could directly react with a dibenzosilole derivative $\mathbf{8 5}$ to produce dibenzo $[g, p]$ chrysene $\mathbf{8 7}$. This K-region-selective reaction was also applicable to pyrene derivatives $\mathbf{8 8}$ to generate multi-annulation products such as hexabenzo[a,c, $f g, j, l, o p]$ tetracenes 89. Interestingly, this efficient $\pi$-annulation also occurred on the bowl-shaped corannulene $\mathbf{1 7}$ to afford phenanthro[9,10- $a]$ corannulene $\mathbf{9 0}$ in $70 \%$ yield [123]. Such a method requires no halogens from the starting PAHs and provides direct access to new PAHs that are difficult to produce by other methods.

In the present section, the overview of synthetic strategies toward PAHs is far from comprehensive. Only the most common methods and representative new approaches have been discussed. New synthetic methods for PAHs are continuously being developed, especially modern transitionmetal catalyzed reactions. Such innovations are expected to further extend the toolbox of PAH synthesis and provide new opportunities for fundamental and applied PAH chemistry.

\section{Vivacity of PAH chemistry in light of GNRs and graphene}

On the one hand, the development of synthetic methods has stimulated the synthesis of various types of PAHs, thereby allowing precise elucidation of structure-property relationships, that is, how the modes of ring fusion, edge structures, "defects," and heteroatom-doping affect the optical, electronic, and magnetic properties of PAHs. On the other hand, research into PAHs has contributed substantially to the bottom-up organic synthesis of GNRs and the interpretation of their properties. From the synthetic point of view, the strategies leading to PAHs can be extended to the fabrication of quasi-1D GNRs, which are, by definition, large PAHs with a high aspect ratio. Furthermore, the molecular precursors
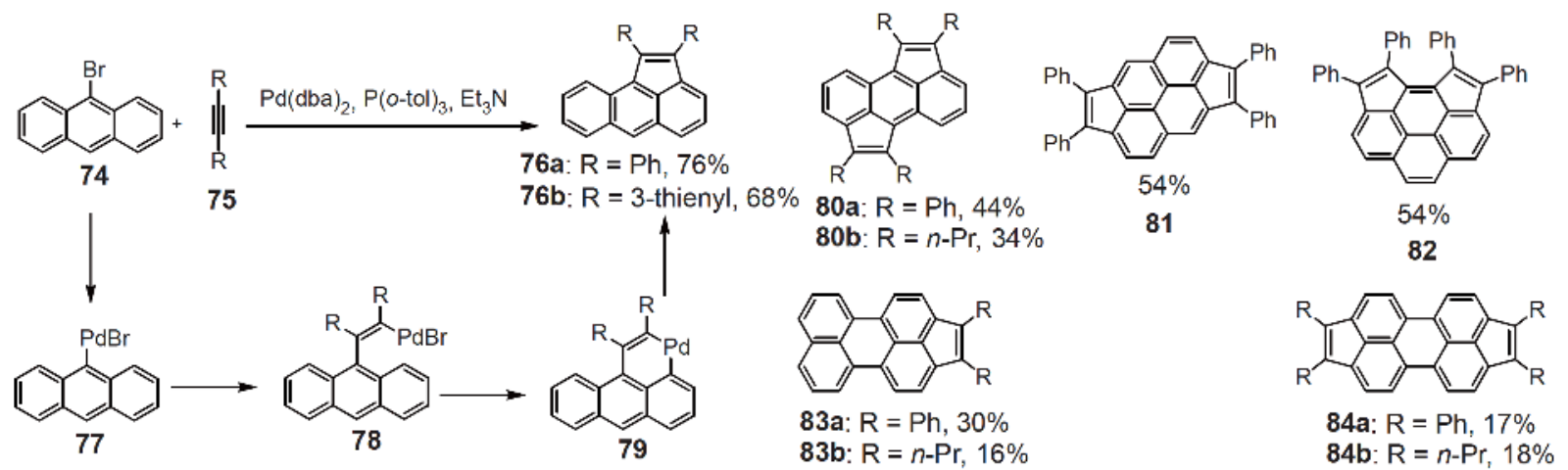

Figure 14 Transition-metal-catalyzed $\pi$-annulation toward cyclopentannelated arenes. $\mathrm{Pd}(\mathrm{dba})_{2}$ : bis(dibenzylideneacetone $)$ palladium $(0)$; $\mathrm{P}(o \text {-tol })_{3}$ : tri $(o-$ tolyl)phosphine; $\mathrm{Et}_{3} \mathrm{~N}$ : triethylamine. 


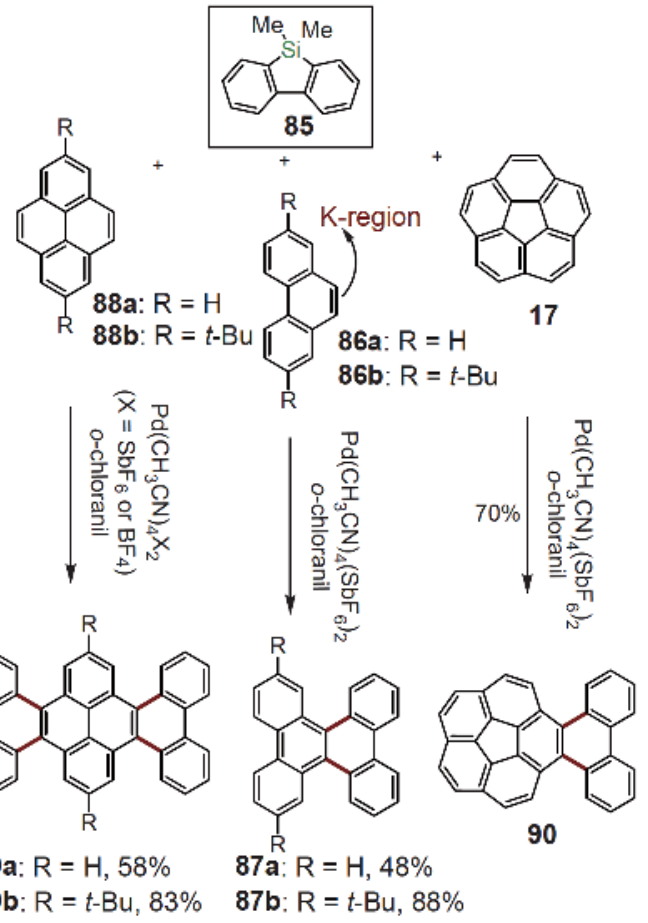

Figure 15 K-region-selective annulative $\pi$-extension through double $\mathrm{C}-\mathrm{H}$ arylation (color online).

used in the synthesis of GNRs are often PAH structures. From the physical point of view, PAHs can serve as model compounds to interpret the properties of GNRs, which can then promote the design of novel GNRs. Therefore, PAHs play an indispensable role in GNR research. Conversely, GNRs raise new motivations for the PAH field, e.g., establishing undeveloped methods for the GNR synthesis, building unprecedented model systems for complex GNR structures, and investigating the properties of defined seg- ments of GNRs. Although PAHs and pristine graphene are too different in size and electronic state diagram, research on defective and heteroatom-doped graphene could acquire new implications from structurally defined PAHs to understand the local geometric, magnetic, and catalytic effects of having defects or heteroatoms in the graphene lattice, and would stimulate the synthesis of new PAH structures that are relevant to such graphene materials. Moreover, the chemistry of PAHs could even expand the knowledge on graphene, for example, with regard to the existence of various edge structures, as discussed later in this section.

The readers are recommended to refer to previous reviews on bottom-up synthesized GNRs for a comprehensive analysis of the history and recent advances $[6,16,62,63,66,67$, $124,125]$. In the following, we only select typical examples to illustrate the close interaction between PAH and GNR chemistries. As described previously, $p$-HBC 6 can be synthesized, first, by formation of the hexaphenylbenzene precursor, followed by oxidative cyclodehydrogenation. By applying this strategy to polymeric systems, GNRs can be fabricated through cyclodehydrogenation of carefully designed polyphenylene precursors. For example, in 2008, Müllen et al. [126] established the solution synthesis of GNRs through $\mathrm{A}_{2} \mathrm{~B}_{2}$-type Suzuki polymerization and subsequent cyclodehydrogenation of the polyphenylene precursor (Figure 16(a)). Similarly, different GNRs have been obtained by $\mathrm{A}_{2} \mathrm{~B}_{2}$-type Diels-Alder polymerization or AAtype Yamamoto polymerization, but the obtained GNRs were still shorter than $100 \mathrm{~nm}$, posing a major challenge to the fabrication of single-ribbon devices [96,127-129]. To address this problem, Narita, Feng and Müllen et al. [130,131] developed an AB-type Diels-Alder polymerization to achieve liquid-phase-processable GNRs longer than $600 \mathrm{~nm}$ (Figure 16(b)). This achievement has not only allowed for

(a)
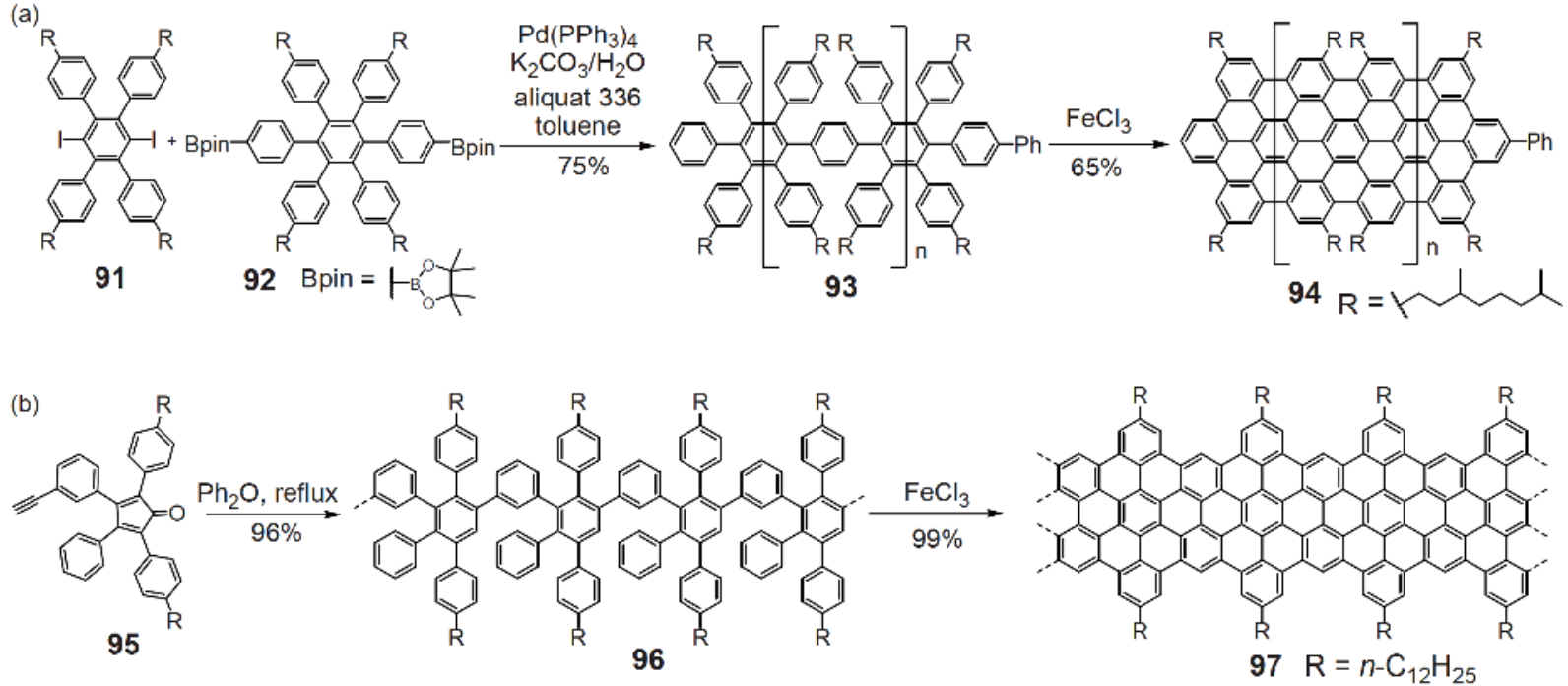

Figure 16 Synthesis of GNRs based on (a) $\mathrm{A}_{2} \mathrm{~B}_{2}$-type Suzuki polymerization and (b) AB-type Diels-Alder polymerization. $\mathrm{Pd}\left(\mathrm{PPh}_{3}\right)_{4}$ : tetrakis(triphenylphosphine)palladium(0). 
the investigation of the fundamental physics of such structurally well-defined GNRs [132-134] but also promoted the fabrication of GNR-based devices by solution processing $[135,136]$. Single-GNR transistor devices demonstrated the semiconducting properties of GNR 97, and thin-film devices exhibited remarkable chemical sensing capability toward $\mathrm{NO}_{2}$ gas with limits of detection down to parts per billion levels [136]. Nevertheless, the charge-carrier mobilities obtained from the GNR-based devices were much lower than the intrinsic values as evaluated by ultrafast terahertz photoconductivity analysis and theoretical calculations $[137,138]$. The poor device performance is presumably due to the high contact resistance between the electrodes and GNRs, requiring improved device fabrication.

The classical solution synthesis has provided a variety of GNRs with different structural features and tunable band gaps, and can afford GNR materials on a gram scale, which can be processed from a solution for physical characterization and device fabrication [127,139-143]. However, proving the structural perfection of such large macromolecules at the atomic level remains a challenging task. Therefore, the on-surface synthesis technique under UHV is valuable not only as an alternative way to synthesize various GNRs but also to in-situ visualize the structure of the obtained GNRs by high-resolution STM and nc-AFM [63]. In 2010, Müllen and Fasel et al. [144] demonstrated the on-surface synthesis of GNRs from dihalogenated monomers, which underwent metal-surface-assisted homocoupling to form the polymer precursors. Subsequent cyclodehydrogenation finally furnished GNRs directly on the metal surface (Figure 17). This work has opened up a new field of on-surface synthesis of atomically precise GNRs. Other synthetic strategies have also been developed to expand the on-surface synthesis toolbox for GNRs. For example, Müllen, Fuchs and Chi et al. [145] employed 1,4,5,8-tetrabromonaphthalene as the mo- lecular precursor to synthesize rylene-type GNRs. Gold-organic hybrids were identified as the intermediate and cyclodehydrogenation was not needed in this reaction. To develop non-halogenated precursors to avoid the contamination of bromine atoms on metal surfaces, Zhang, Müllen and Chi et al. [146] reported a new pathway to rylene-type GNRs by exploring perylene-3,4,9,10-tetracarboxylic dianhydride (PTCDA) as the precursor on $\mathrm{Cu}$ (111) surfaces. Similar to the previous example, copperperylene chains were observed as the intermediate after decarboxylation of PTCDA, and the GNRs were subsequently generated by annealing at a higher temperature. Another method that does not require halogenated precursors was reported by $\mathrm{Hu}$ and $\mathrm{Xu}$ et al. [147] through Bergman cyclization. The obtained diradical species readily underwent polymerization to afford naphthalene-substituted polyphenylenes on the surface. Although further cyclodehydrogenation was not demonstrated in this study, such a protocol is in principle feasible to synthesize GNRs with carefully designed precursors. Despite the success in onsurface synthesis of GNRs under UHV, the relatively high cost of the UHV setup and low scalability of the on-surface synthesis under UHV limit its potential for practical applications. To address this issue, the CVD technique was used to develop an industrially viable on-surface synthesis under low-vacuum or even ambient pressure [148,149]. Large areas of GNR films can thus be grown by this method, which is technologically important in terms of large-scale fabrication and facile device integration.

Both in-solution and on-surface synthesis techniques have allowed for the bottom-up synthesis of various GNRs and the investigation of their properties. What is crucial for this research philosophy is that such advances cannot be isolated from the development of PAHs. As both fields are growing rapidly, we will select representative examples to illustrate

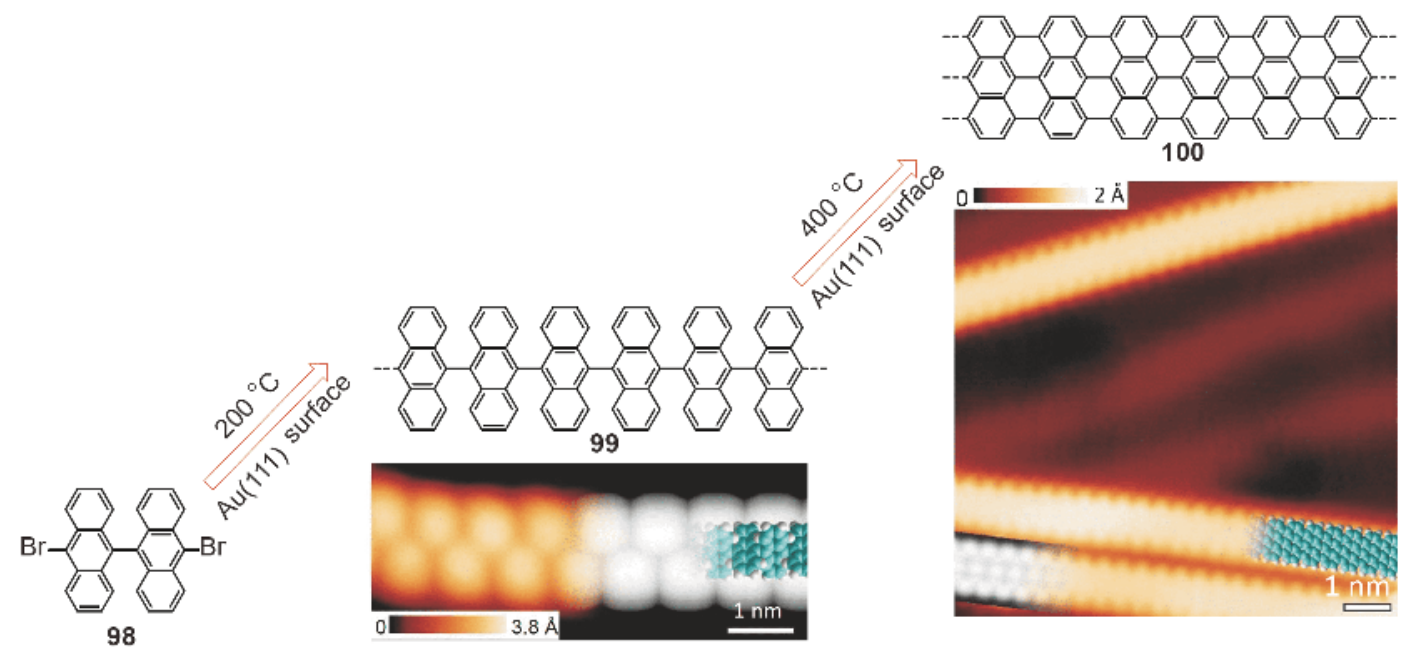

Figure 17 On-surface synthesis of GNRs via polymerization of dihalogenated precursors and subsequent cyclodehydrogenation. STM images are reprinted with permission from Ref. [144], copyright (2010) Macmillan Publishers Ltd. (color online). 
their close relation and synergies.

One might wonder whether 2D graphene could be made via the bottom-up methods as accomplished for GNRs. Certainly, many established methods are available for graphene fabrication (see previous reviews [3-9]), such as topdown exfoliation starting from graphite [150-153], as well as bottom-up CVD synthesis and epitaxial growth on $\mathrm{SiC}$ [154157]. A great achievement for organic chemists would be the synthesis of a giant 2D graphene via defined organic reactions, e.g., carbon-carbon coupling. Indeed, such a synthetic method was demonstrated by Chen and $\mathrm{Hu}$ et al. [158] in 2013 by using hexabromobenzene $\mathbf{1 0 1}$ as the precursor (Figure 18(a)). The breaking of the $\mathrm{C}-\mathrm{Br}$ bonds generated radical intermediates $\mathbf{1 0 2}$, which polymerized on a $\mathrm{Cu}(111)$ surface in $2 \mathrm{D}$ to furnish graphene films at a low temperature of $220-250{ }^{\circ} \mathrm{C}$. This idea of coupling molecular precursors rather than assembling carbon species decomposed from gaseous or solid precursors at high temperatures, as observed in the classical CVD process $[38,156,159]$, has also been explored by $\mathrm{Xu}$ et al. [42]. In 2012, they employed PAHs, including rubrene $\mathbf{1 0 3}$, pentacene $\mathbf{1 0 4}$, and coronene 43 , as precursors in the CVD synthesis of graphene through dehydrogenative coupling (Figure 18(b)). The quality of the resulting graphene films, as judged by Raman spectroscopy, was largely dependent on the different PAH precursors, with coronene providing the best quality at a low growth temperature of $550{ }^{\circ} \mathrm{C}$. From a technological viewpoint, different choices exist for the large-scale fabrication of graphene; nevertheless, PAH chemistry has provided a new angle to the bottom-up synthesis of graphene.

\subsection{Edge topology engineering}

PAHs with different edge topologies exhibit considerably different physical and chemical properties. Typical edge structures of PAHs (Figure 19) include bay regions (also known as armchair edges), L-regions (zigzag edges), K-regions (isolated carbon-carbon double bonds that do not belong to the Clar sextet), as well as cove and fjord regions that cause non-planarity due to the prevailing steric hindrance. The variety of edge topologies enables the control of PAH properties by "edge design."

Such edge topology engineering also plays a pivotal role in GNRs and is only possible through bottom-up synthesis. Armchair GNRs (AGNRs) and zigzag GNRs (ZGNRs) are two representative cases that have attracted the most interest in the GNR field (Figure 20(a, b)). Their width can be defined by the number $(N)$ of carbon atoms across the ribbon. According to their different widths, AGNRs can be divided into three groups with $N=3 n, 3 n+1$, and $3 n+2$ ( $n$ is an integer). The band gap in each group decreases as the ribbon width increases, whereas in different groups but with the same $n$, the band gap is dependent on the group ( $3 n$
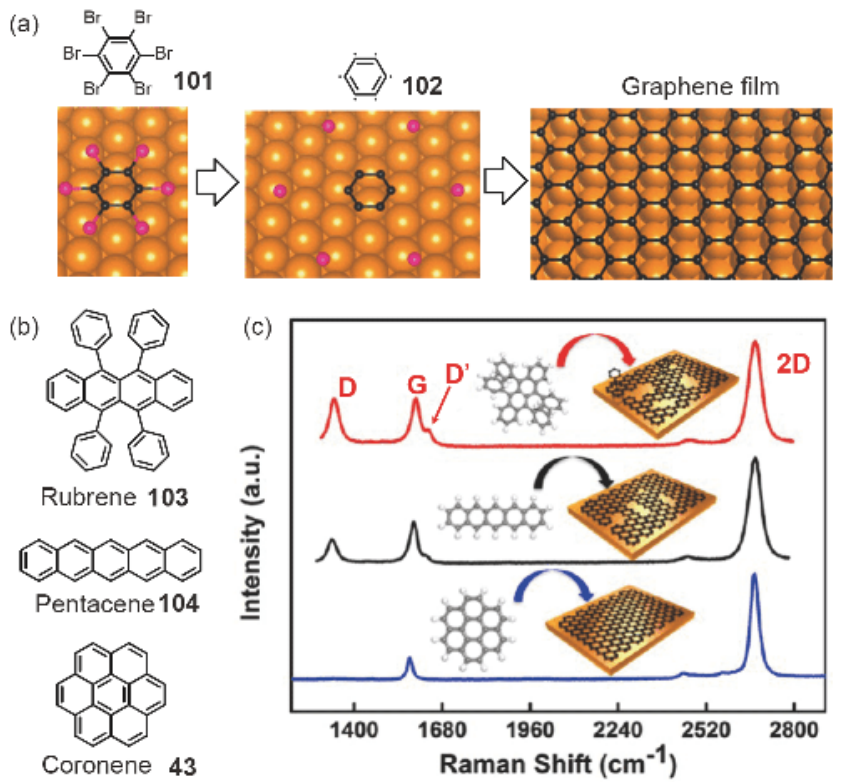

Figure 18 (a) Synthesis of graphene via 2D polymerization of hexabromobenzene. Reprinted with permission from Ref. [158], copyright (2013) American Chemical Society. (b,c) Synthesis of graphene via dehydrogenative coupling of different PAH precursors (b) and the corresponding Raman spectra of the resulting graphenes (c). Reprinted with permission from Ref. [42], copyright (2012) American Chemical Society (color online)

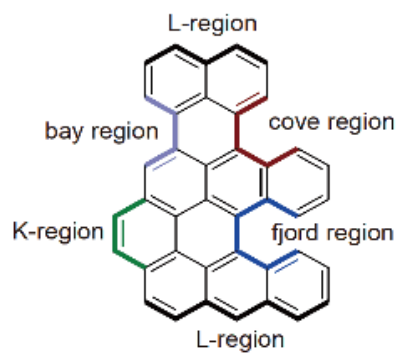

Figure 19 Schematic representation of typical edge topologies of PAHs (color online)

$+2 \ll 3 n<3 n+1$ ) that the GNRs belong to (Figure 20(c)) $[22,160]$. In recent years, AGNRs with different widths have been precisely synthesized via the bottom-up procedure [126,140,142-146,149,161-170], establishing the widthdependent band gaps as determined by theory [22,171]. ZGNRs are predicted to have localized edge states that can be spin-polarized, showing great potential for spintronic applications [160,172]. Furthermore, ZGNRs narrower than $7 \mathrm{~nm}$ are antiferromagnetic with antiparallel spin alignment on the opposite edges of the ribbon, and semiconducting with their band gaps inversely proportional to their widths. On the other hand, ZGNRs wider than $8 \mathrm{~nm}$ exhibit a sharp transition to a ferromagnetic (with magnetic moments on opposite edges aligned in a parallel manner) and metallic (with a vanishing band gap) state (Figure 20(d)) [160]. In 2016, Müllen and Fasel et al. [173] synthesized the first atomically 
(a)

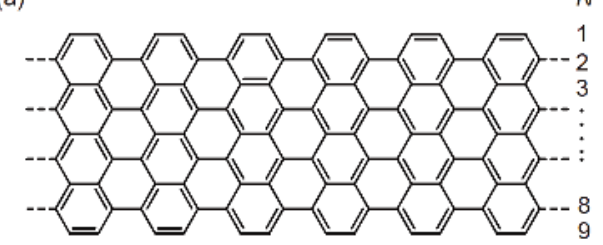

$N=9$ armchair GNR

(c)

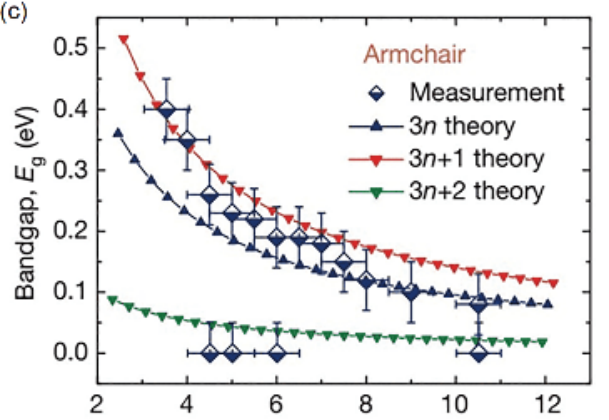

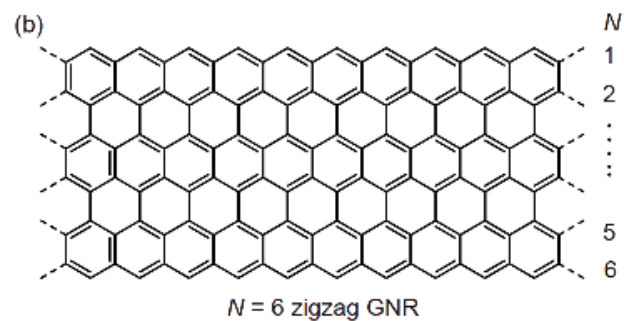

(d)

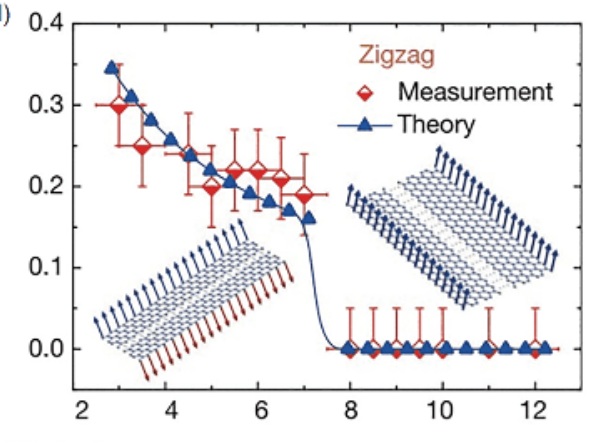

Ribbon width (nm)

Figure 20 (a, b) Chemical structures of $N=9$ armchair GNRs (9-AGNRs) and $N=6$ zigzag GNRs (6-ZGNRs) with illustration of width $N$; (c, d) band gaps in AGNRs and ZGNRs as a function of ribbon widths. Reprinted with permission from Ref. [160], copyright (2014) Macmillan Publishers Ltd. (color online).

precise ZGNR under UHV, demonstrating the localized edge states as anticipated. For 2D graphene, controlling the edge topology with atomic precision through the available fabrication methods is nearly impossible, but one could easily learn lessons from the research on structurally well-defined PAHs and GNRs, such as regarding the stability of armchair edges and the high reactivity of zigzag edges $[174,175]$.

An exemplary case to illustrate the essence of edge topology engineering of PAHs is the edge extension of $p$-HBC 6, which is a fully benzenoid PAH with all-armchair edges. Modulation of the electronic properties becomes possible by fusing additional benzene rings onto the $p$-HBC core (Figure $21)$. These extra K-regions drastically change the photophysical characteristics, as evidenced by prominent red shifts of the absorption maxima when going from $p$-HBC to $\pi$ annelated derivatives 105, 106, and 107. Recently, $p$-HBC with four additional K-regions 108, which generate two parallel zigzag edges and four relatively short zigzag edges, was synthesized by Feng and Müllen et al. [176], displaying a significantly lowered optical gap compared with $p$-HBC. By adding six K-regions to $p$-HBC, compound 109 with a full zigzag periphery has attracted much theoretical interest but remains elusive. However, by employing 6,13-bis(10bromoanthracen-9-yl)-1,4,8,11-tetramethylpentacene $\mathbf{1 1 0}$ and 10,10'-dibromo-9,9'-bianthracene $\mathbf{9 8}$ as the monomers for on-surface synthesis (Figure 22(a)), the structure motif of 109 was embedded into 7-AGNRs, generating topologically non-trivial quantum phases. As indicated by scanning tunneling spectroscopy (STS) and STS mapping, a new quantum state at the interface of 7-AGNRs and edge-extended sections featuring the structural motif of $\mathbf{1 0 9}$ was observed

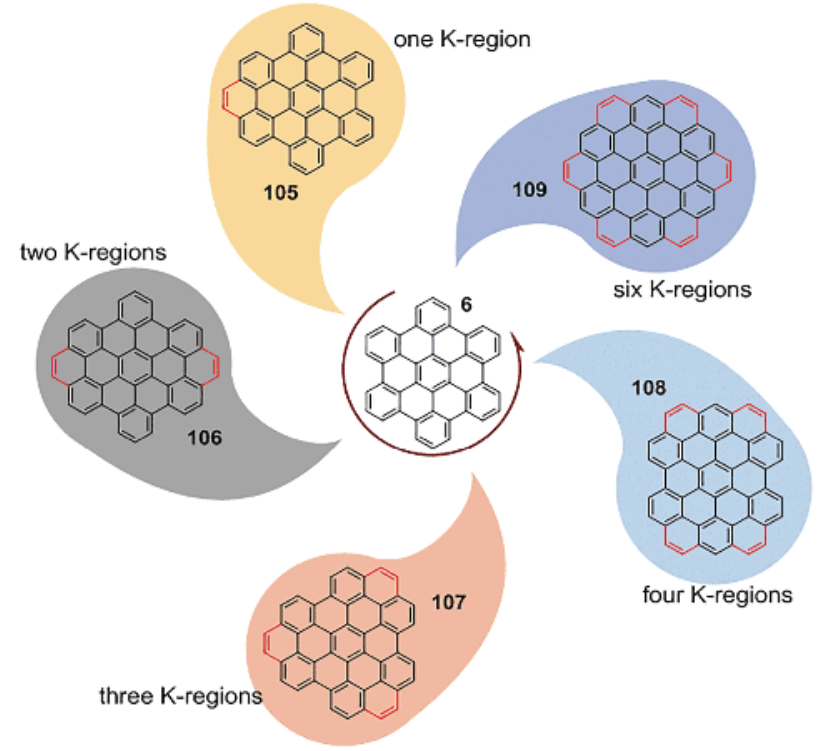

Figure 21 Modulation of electronic properties of $p$-HBC by adding one, two, three, four, and six K-regions (color online).

because of their topological non-equivalence (Figures 22(b, c)) [177]. Another relevant work on GNR-based topological insulators was based on the fabrication of 7/9-AGNR heterojunctions (Figures 22(d-f)), which, from the structural point of view, were formed by partially adding K-regions to the armchair edge of 7-AGNRs [178]. Such an edge extension, which was first developed in PAH systems, has greatly stimulated the design of new GNRs such as emerging topological insulators.

The majority of PAHs have armchair structures, whereas 


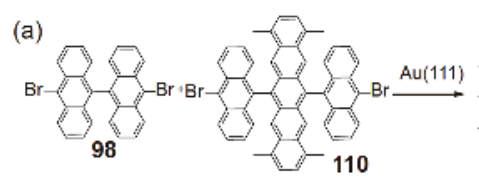

(b)

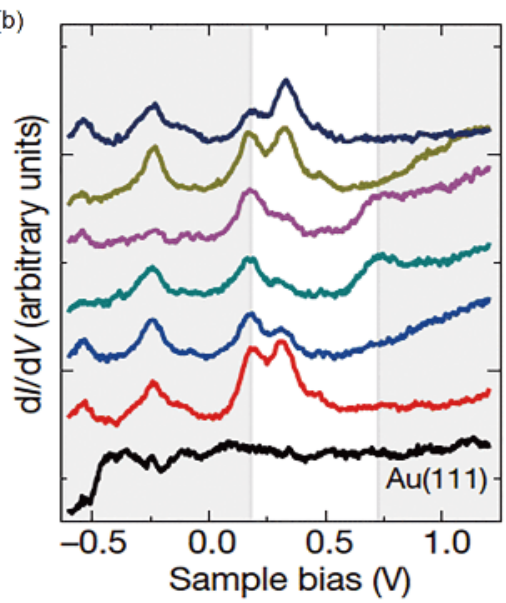

(c)

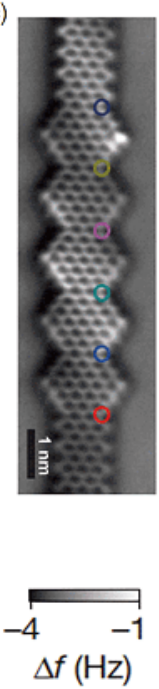

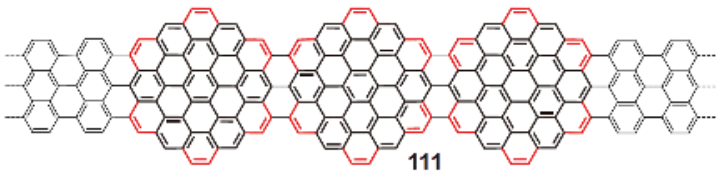

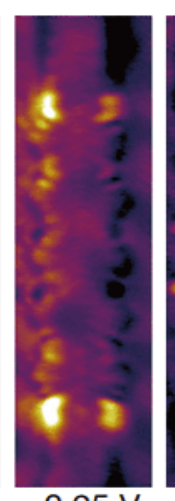

$0.25 \mathrm{~V}$

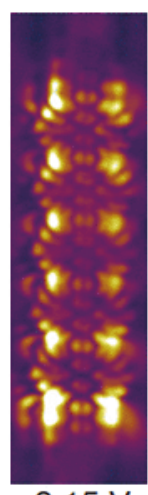

$0.15 \mathrm{~V}$

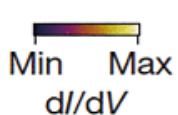

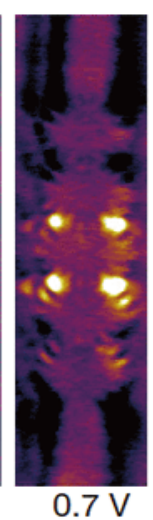

$0.7 \mathrm{~V}$
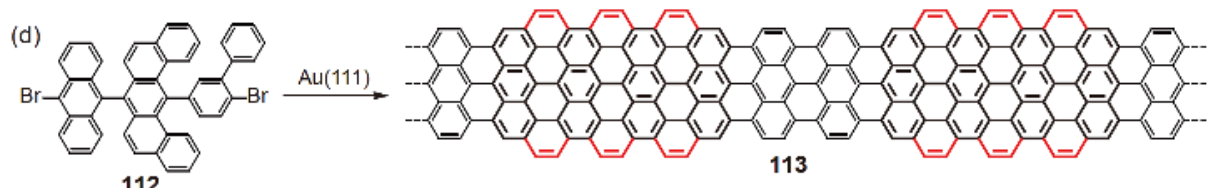

(e)
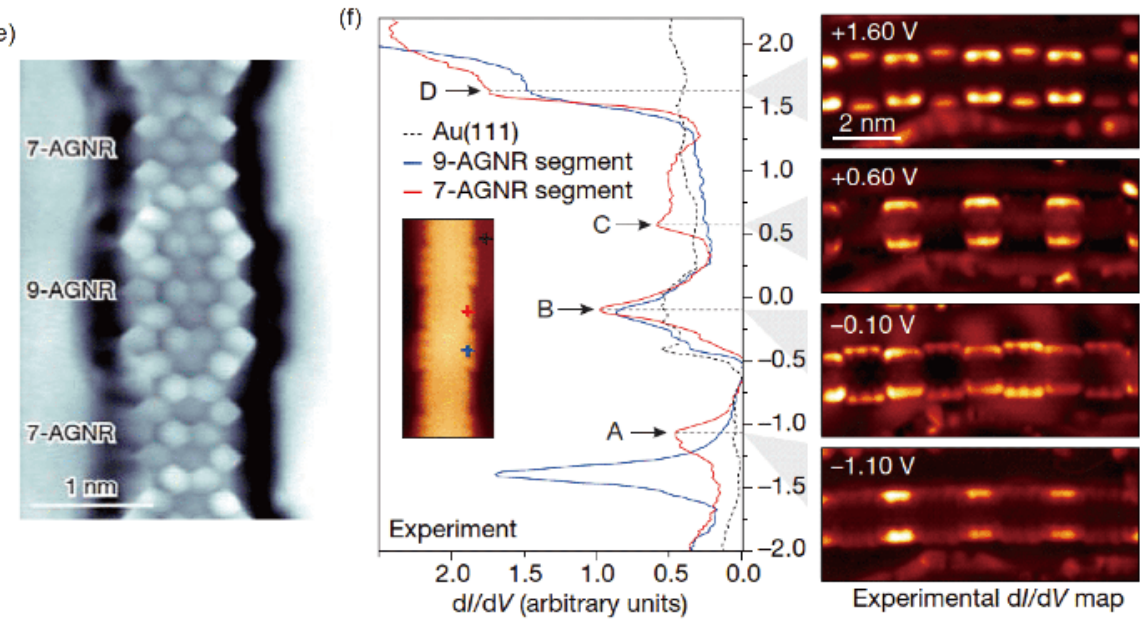

Figure 22 (a) Surface-assisted synthesis of GNR 111 using 98 and $\mathbf{1 1 0}$ as precursor molecules. (b) $\mathrm{d} / / \mathrm{d} V$ spectra taken at locations indicated by corresponding color markers in panel c. (c) Constant-height nc-AFM image of GNR 111 and experimental d $/ / \mathrm{d} V$ maps of GNR 111 on Au(111). (d) Surfaceassisted synthesis of GNR 113 from molecular precursor 112. (e) Bond-resolved STM image of 7/9-AGNR superlattice on Au(111). (f) d $I / \mathrm{d} V$ spectra of GNR 113 taken at locations indicated by corresponding color markers, and constant-current $\mathrm{d} / / \mathrm{d} V$ maps of GNR 113 on Au(111). (b, c) Reprinted with permission from Ref. [177], copyright (2018) Macmillan Publishers Ltd.; (e, f) reprinted with permission from Ref. [178], copyright (2018) Macmillan Publishers Ltd. (color online).

zigzag-edged PAHs are relatively rare. Acenes are a unique class of PAHs featuring long zigzag edges [179]. Their structures are simply composed of benzene rings fused in a linear mode (Figure 23), whereas their syntheses are challenging, especially for higher homologues longer than pentacene 104. Pentacene is a prototypical semiconductor whose application greatly suffers from its high tendency toward oxidation or photodimerization. This high chemical reactivity is pertinent to the long zigzag edges, which favors the formation of diradical ground states (Figure 23(a)), and the driving force to form stable oxidized or dimerized products $[180,181]$. The instability issue is even more severe for higher acenes, obstructing their syntheses and applications. Thus, Müllen et al. [182] developed a precursor route to 


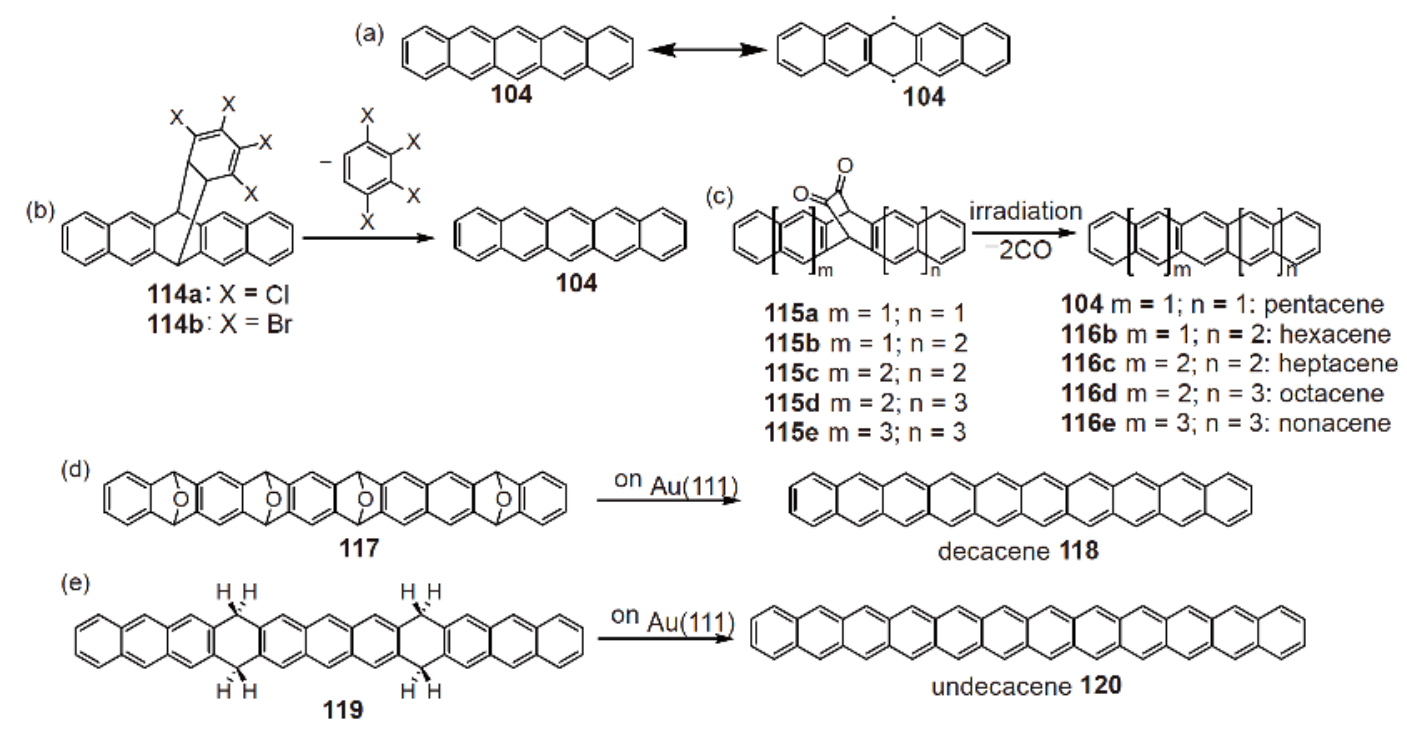

Figure 23 The family of linear acenes and their different synthetic strategies. (a) Pentacene and its diradical resonance structure; (b) synthesis of pentacene via retro-Diels-Alder reactions in the solid state; (c) synthesis of higher acenes up to nonacene 116e in polymer matrices; (d, e) surface-assisted synthesis of decacene 118 and undecacene 120.

convert the stable and soluble precursors 114 into pentacene by retro-Diels-Alder reactions in the solid state (Figure 23 (b)). Similarly, Neckers et al. and Bettinger et al. [183-185] achieved a series of higher acenes up to nonacene 116e in polymer matrices by photochemical extrusion of bridging groups from the ambient-stable precursors 115e (Figure 23 (c)). The solid-state reactions directly provided the highly reactive product in an inert environment, thereby solving the instability problem. Another way to achieve longer acenes relies on the surface-assisted chemical reactions under UHV, which prevents the contact of the sensitive product with oxygen. The metal surfaces also stabilize the highly reactive acene species. A similar "extrusion" concept was applied by Moresco and Peña et al. [186] to obtain decacene 118 through surface-assisted reduction of a tetraepoxy decacene precursor 117 (Figure 23(d)). The highest homologue undecacene 120 was realized in 2018 by Godlewski and Echavarren et al. [187] via on-surface dehydrogenation of a partially saturated precursor 119 (Figure 23(e)).

When acenes are fused laterally at all peri-positions, a new class of PAHs called peri-acenes is formed (Figure 24). The smallest member of the family is perylene $\mathbf{3 9}$ and the next larger one is bisanthene 125. Both compounds are closedshell structures that have been known for decades [188-191], whereas the higher homologues peri-tetracene $\mathbf{1 2 6}$ and peripentacene 127 start to exhibit open-shell diradical ground states (Figure 25(a)), thereby posing challenges for syntheses and characterizations. After a long pursuit of these structures, Liu and Feng et al. [192] (Figure 25(b)) and Wu et al. [193] independently synthesized different peri-tetracene derivatives $\mathbf{1 3 4}$ in 2018. Although the synthesis of peri-pentacene $\mathbf{1 2 7}$ has not been successful in solution, it has been demonstrated by Crommie and Fischer et al. [194] through on-surface cyclodehydrogenation of 6,6'-bipentacene $\mathbf{1 3 5}$ under UHV (Figure 25(c, d)).

Further extension of peri-acenes along the peri-positions (along the $Y$ axis in Figure 24) provides further opportunities for new PAH-based materials, and eventually, gives rise to a series of AGNRs (5-, 7-, 9-, 11-, 13-AGNRs, and so on). Therefore, the PAHs shown in Figure 24 are suitable model compounds for these GNRs. When the rylene molecules become longer (such as terrylene $\mathbf{1 2 8}$ and quaterrylene 130), their HOMO-LUMO gaps decrease significantly, resulting in a band gap as low as $0.1 \mathrm{eV}$ and a metallic or half-metallic behavior when reaching the infinite 5-AGNRs $[163,166,195]$. Theoretical simulations have suggested the appearance of singlet edge states when the length of the rylene ribbon is longer than 7 perylene monomers [166], whereas experimental studies on a series of cyclopenta ringfused rylene compounds have revealed substantial open-shell singlet diradical character from hexarylene (3 perylene monomers) onward [196]. These results have indicated the existence of localized edge states at the termini of 5-AGNRs. Furthermore, rylenes can be stabilized by attaching auxochromic groups at their peri-positions, generating an important family of chromophores which serve not only as industrial colorants but also as key materials for bioimaging, organic optoelectronics, and single-molecule spectroscopy [48]. The longer versions of bisanthene 125, e.g., teranthene 129, and quateranthene 131, have been proven to possess unpaired electrons localized at the zigzag edges (diradical ground states) in contrast to the closed-shell bisanthene $[197,198]$. These findings are in accordance with the observation of giant edge state splitting at the termini of 7- 


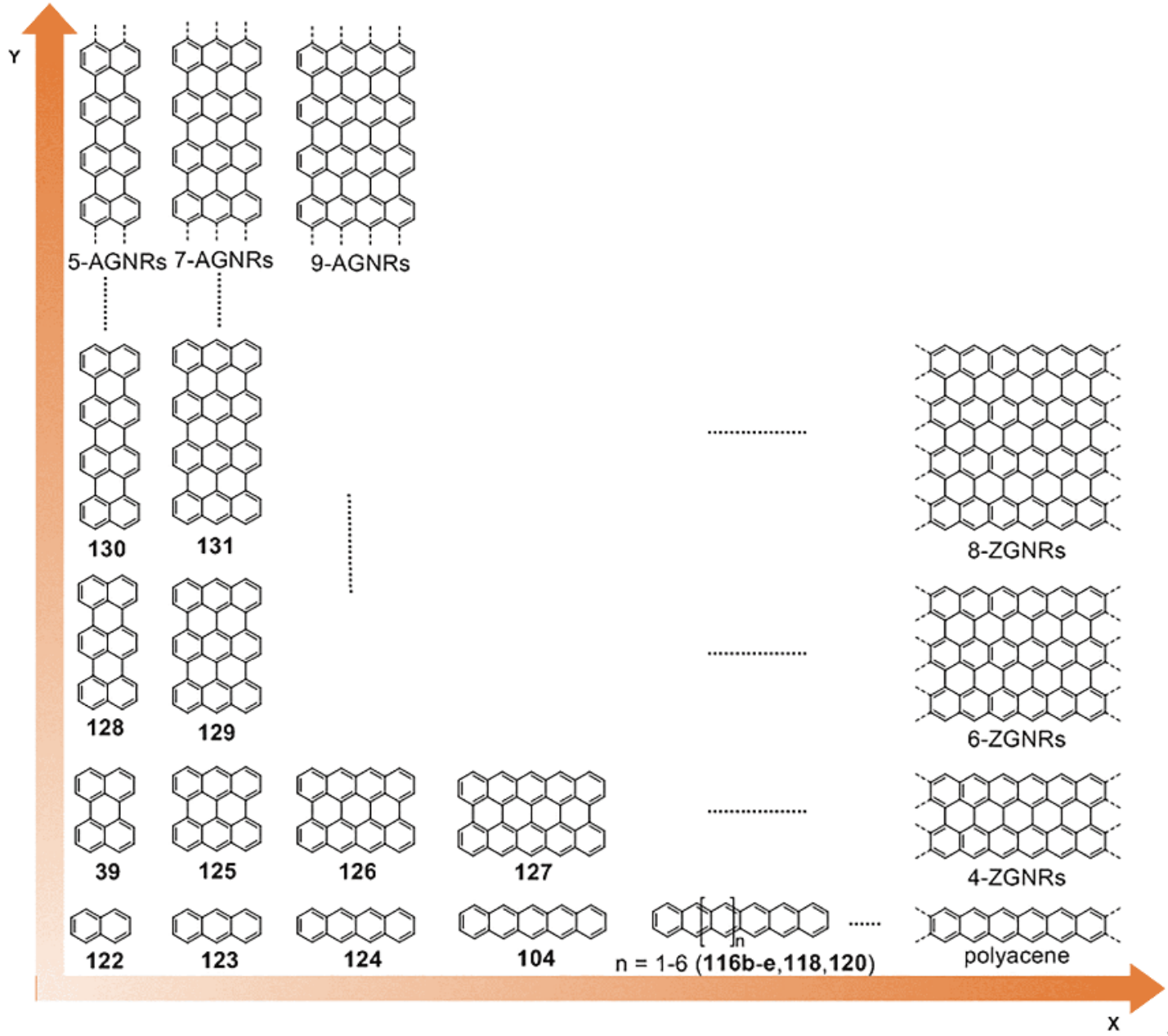

Figure 24 Extension of acenes along different dimensions leading to a variety of PAHs and GNRs (color online).
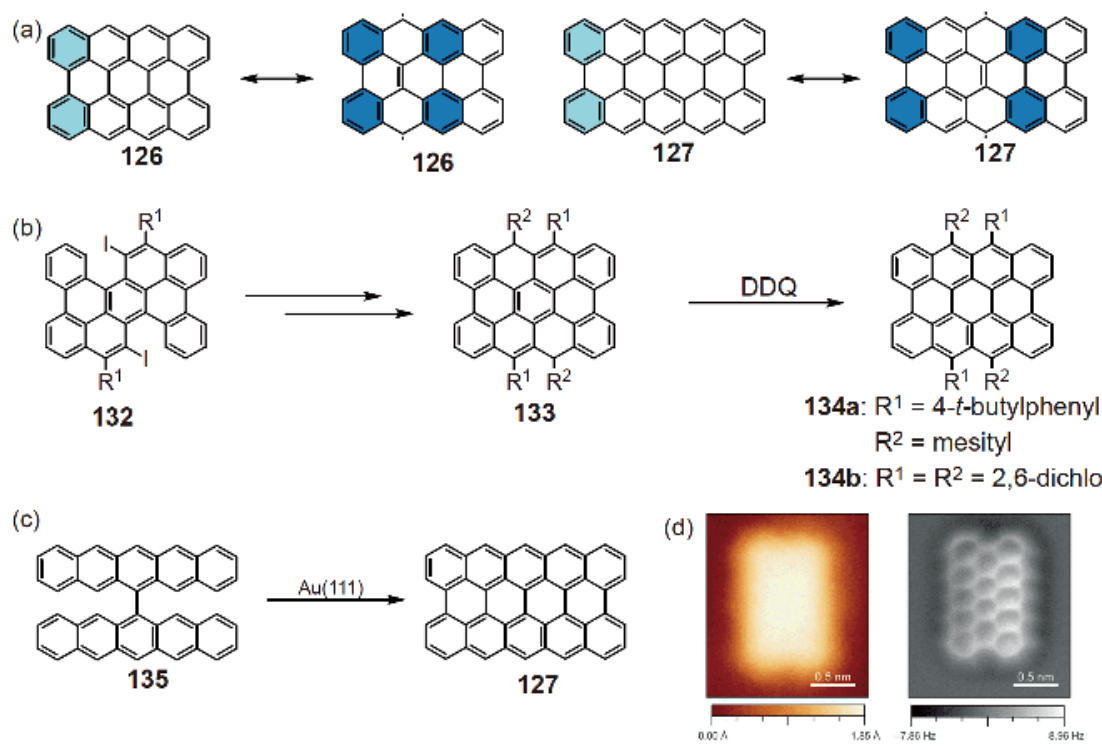

Figure 25 (a) Resonance structures of peri-tetracene 126 and peri-pentacene 127; (b) synthesis of peri-tetracene derivatives 134 in solution; (c) surfaceassisted synthesis of peri-pentacene; (d) high-resolution STM image and constant-height nc-AFM image of peri-pentacene on Au(111). DDQ: 2,3-dichloro5,6-dicyano-1,4-benzoquinone. Reprinted with permission from Ref. [194], copyright (2015) Wiley (color online). 
AGNRs [199,200]. As shown in Figure 24, the longer homologues of peri-tetracene are still missing, but one could learn from the significant open-shell diradical character of peri-tetracene that 9-AGNRs would also possess edge states if the termini maintain the zigzag topology as in peri-tetracene. This is, however, not the case in an on-surface synthesized 9-AGNR, in which no localized edge states have been observed at the termini because of the armchair-type termination [161]. Such a difference suggested that not only the width and edge structures matter for the properties of GNRs but also the terminus topology. If the rylene compounds are extended along the $X$-axis (Figure 24), they will end up in a series of ZGNRs with a full zigzag edge topology (4-, 6-, 8-, 10-ZGNRs, and so on). Considering the model compounds, such as long acenes and peri-acenes, organic chemists can easily predict a high reactivity and, thus, instability of ZGNRs under ambient conditions, making it extremely difficult to synthesize ZGNRs by conventional solution chemistry. The instability issue is not a problem for on-surface synthesis under UHV, which prevents the oxidation side reactions and stabilizes the conjugated structure by interaction with the metal surface. However, the on-surface synthesis of ZGNRs is not straightforward, because the zigzag edge structure cannot be constructed through conventional surface-assisted benzene-benzene couplings, which only lead to armchair edges. Thus, a new design concept of the monomer was needed to synthesize the challenging ZGNRs, which had been predicted to possess magnetic edge states along the zigzag edges. In 2016, Müllen and Fasel et al. [173] achieved the long-awaited atomically precise ZGNRs from a carefully designed umbrella-shaped monomer 136 with a short zigzag edge and methyl groups that underwent oxidative cyclization with the neighboring benzene rings to form full zigzag edges (Figure 26(a)). It should be emphasized that monomer $\mathbf{1 3 6}$ itself is a PAH, which required a 14-step organic synthesis, highlighting the indispensable role of demanding synthetic chemistry. The structure of the obtained 6-ZGNRs $\mathbf{1 3 8}$ has been verified by STM and nc-AFM, and the theoretically predicted localized edge states along the zigzag peripheries have been experimentally confirmed by STS measurements (Figure 26(b-d)). The 6-ZGNR is the only atomically precise ZGNR; therefore, ZGNRs should be explored with other widths to investigate the width-dependent properties [160]. Further, as shown in Figure 24, there are many elusive, but certainly challenging, PAHs through extension of acenes along different dimensions. These are interesting molecules in their own right and also important for understanding the physical properties of their corresponding GNRs.

PAHs with long zigzag edges are often prone to oxidation due to their open-shell diradical character. Although strategies, such as kinetic protection by bulky substituents, are applied to stabilize these compounds, the instability issue

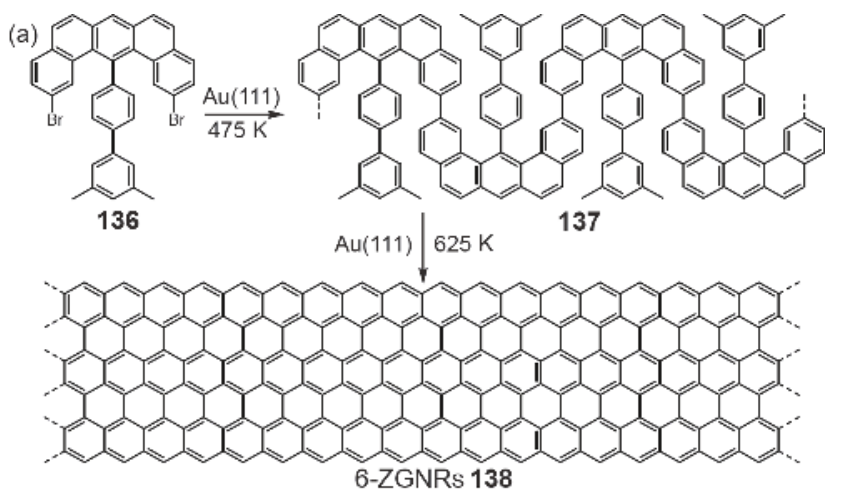

(b)

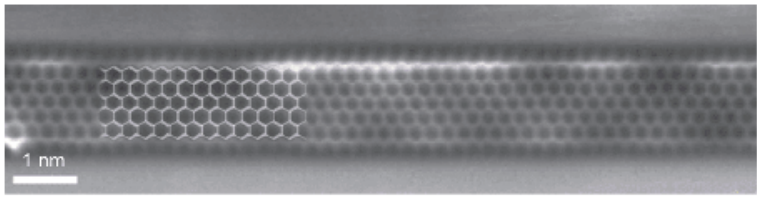

(c)
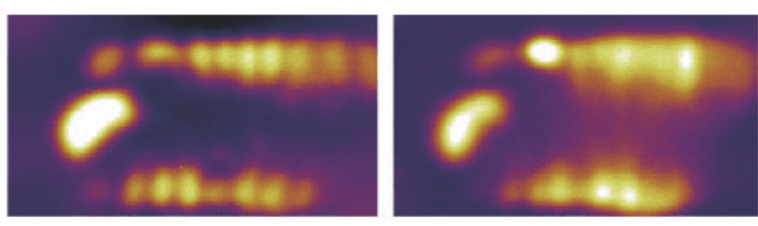

(d)

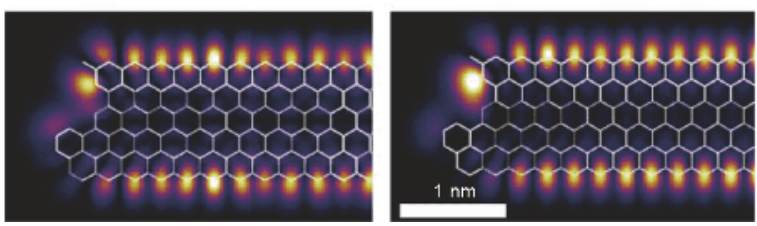

Figure 26 (a) Surface-assisted synthesis of 6-ZGNRs 138; (b) constantheight nc-AFM image of 6-ZGNRs on $\mathrm{Au}(111)$ taken with a CO-functionalized tip; (c) differential-conductance maps of filled (left) and empty (right) edge states of 6-ZGNRs on $\mathrm{Au}(111)$; (d) local density of states showing the spatial distribution of filled (left) and empty (right) edge states. Reprinted with permission from Ref. [173], copyright (2016) Macmillan Publishers Ltd. (color online).

cannot be fully set aside, and thus limits further investigations and applications. In this regard, stable PAHs featuring zigzag edges, but without biradical ground states, have also been investigated. For instance, Müllen, Narita and Scotognella et al. [201] reported the synthesis and properties of dibenzo[hi,st] ovalene 142 (Figure 27), which exhibited high stability under ambient conditions and strong red emission with a quantum yield of up to $79 \%$. This compound also showed stimulated emission and amplified spontaneous emission. These properties indicate the potential of such stable zigzag-edged PAHs as a new type of structurally defined GQDs for applications in organic light-emitting diode (OLED) and laser devices. In this sense, GNRs with a partial zigzag edge topology would combine attractive electronic properties with sufficient stability.

Armchair and zigzag edges are considered as the two prevailing edge configurations in graphene research [174, 175,202-204]. However, by visiting the PAH chemistry, one could imagine that more diverse edge topologies of graphene 
exist. For example, the cove region as observed in [4]helicene (with the number indicating how many benzene rings are ortho-fused together, see Figure 28(a)) endows the PAHs with non-planarity [54-57]. A representative example is contorted $c$-HBC 29a featuring six cove regions, as first synthesized by Clar et al. [205] in 1965 and later by Nuckolls et al. $[95,206]$ with a new method and improved yields (Figure 28(a)). In 2015, Feng and Müllen et al. [207] reported the synthesis of the first cove-edged GNRs 144 (Figure 28(a)). A series of oligomers were synthesized in solution, revealing non-planar edge geometries by single- crystal X-ray analysis, and longer GNR homologues were obtained via on-surface synthesis under UHV. Furthermore, PAHs containing the [5] helicene $\mathbf{1 4 5}$ substructure exhibit the fjord region (Figure 28(b)), as exemplified by the incompletely cyclized $p$-HBC analogs, such as hexabenzoperylene 146 and hexabenzotriphenylene 147 [208,209]. The latter two cases are also known as double [5] helicene and triple [5] helicene, respectively. Even more complex PAHs containing helicene moieties have been synthesized [210,211], such as a hexapole [5]helicene $\mathbf{1 4 8}$ (Figure 28(b)) [212,213], a double [6]helicene 149 (Figure

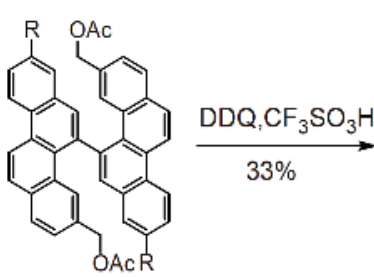

139

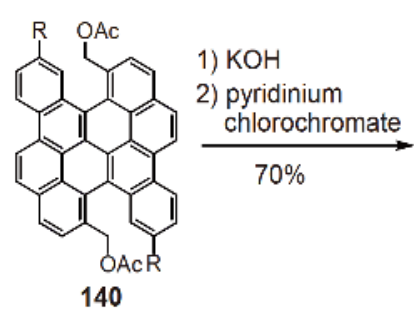

140

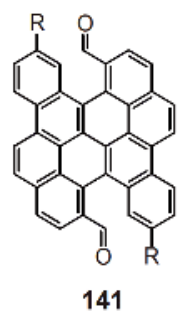

141

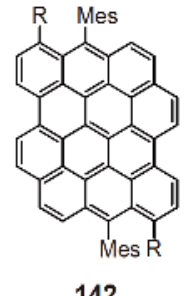

$\mathrm{R}=$ alkyl chains

Figure 27 Synthesis of dibenzo[hi,st] ovalene 142. DDQ: 2,3-dichloro-5,6-dicyano-1,4-benzoquinone.

(a)<smiles>c1ccc2c(c1)ccc1ccc3ccccc3c12</smiles>
[4]helicene 143

(b)

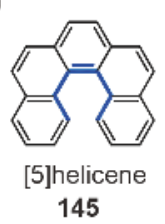

145

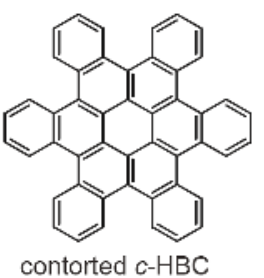

29a

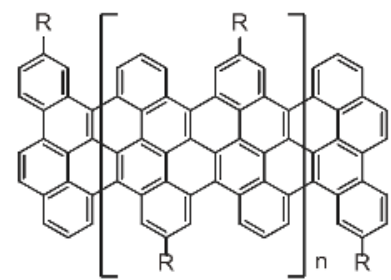

cove-edged GNRs 144 ( $\mathrm{n}=0,2,3,6 ; \mathrm{R}=\mathrm{H}$ or $t$-Bu)
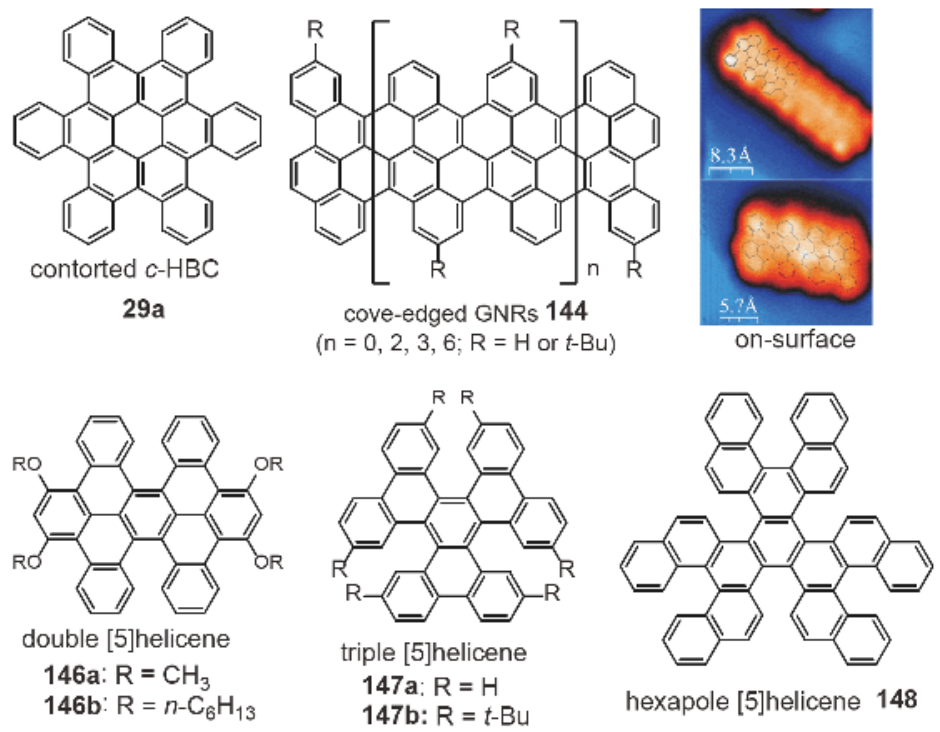

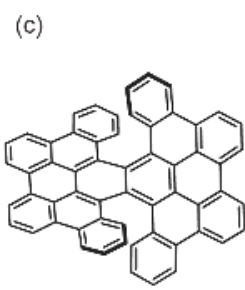

double [6]helicene

149

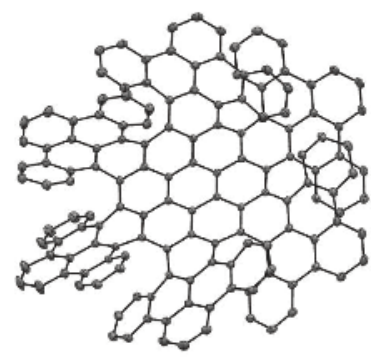

hexapole [7]helicene 150

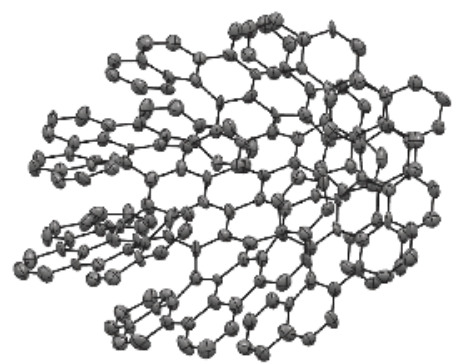

hexapole [9]helicene 151

Figure 28 (a) Examples of PAHs and GNRs with cove regions (containing [4]helicene moieties); (b) PAHs with fjord regions (containing [5] helicene moieties); (c) PAHs containing [6], [7] and [9] helicene moieties. The STM image in (a) was reprinted with permission from Ref. [207], copyright (2015) American Chemical Society (color online). 
28(c)) [214], as well as a hexapole [7]helicene 150 and a hexapole [9] helicene $\mathbf{1 5 1}$ based on the $p$-HBC core (Figure 28(c)) $[215,216]$. Such remarkable synthetic achievements of PAH chemistry offer new insights for a detailed discussion of the graphene edge topologies. Moreover, the growing interest in such helicene-containing PAHs has further stimulated the synthesis of helical nanographene molecules [217-219] and even GNRs [220].

\subsection{Edge functionalization}

Functionalization of graphene is important to modulate its electronic properties and allow for new coupling reactions. Various methods have been developed to covalently functionalize pristine graphene and can be generally divided into (1) radical additions to the $\mathrm{sp}^{2}$-carbons of graphene (e.g., by using a diazonium salt) and (2) cycloadditions to the carboncarbon double bonds (e.g., by using an azomethine ylide, nitrene, or aryne species) [202,221-223]. Graphene oxide (GO), the oxidized form of graphene, can also be functionalized by exploring the chemical reactions of the existing oxygen-containing groups, including hydroxyl $(-\mathrm{OH})$, carboxyl $(-\mathrm{COOH})$, and epoxy units [224]. GO is usually produced by the Hummers method, that is, treating graphite with strong chemical oxidants (e.g., sodium nitrate, sulfuric acid, and potassium permanganate), followed by exfoliation into layers of graphite oxide [225]. The strong oxidation conditions uncontrollably generate different oxygen-containing functional groups at the edge and on the basal plane. By contrast, the oxidation of molecular nanographenes is able to selectively provide edge-oxidized structures. For example, Müllen et al. [226] reported the selective oxidation of the Kregion of $\pi$-extended $p$-HBC derivative $\mathbf{1 5 2}$, leading to the formation of an $\alpha$-diketone moiety, which was used as a building block to synthesize large heteroatom-containing PAHs such as $\mathbf{1 5 4}$ (Figure 29). This defined oxidation would also be desirable for graphene chemistry.

Another type of graphene functionalization relates to the attachment of hydrogen or halogen atoms to $\mathrm{sp}^{2}$-carbons of graphene, generating $\mathrm{sp}^{3}$-hybridized carbon centers and thus opening the band gap of graphene due to the interruption of $\pi$-delocalization [221]. Partially hydrogenated graphene exhibits a tunable band gap and ferromagnetism, whereas the fully hydrogenated graphene, which is known as graphane, behaves as an insulator while maintaining hexagonal symmetry $[227,228]$. A similar trend holds true for halogenated graphene, although only the fully fluorinated graphene (stoichiometric graphene fluoride, $\mathrm{C}_{1} \mathrm{~F}_{1}$ ), can be obtained among the different graphene halides [229-230].

Hydrogenated graphene can be achieved either by gasphase hydrogenation through exposing graphene to a hydrogen plasma [231,232], or by liquid-phase reaction, e.g., Birch reduction in the presence of lithium/sodium and al-
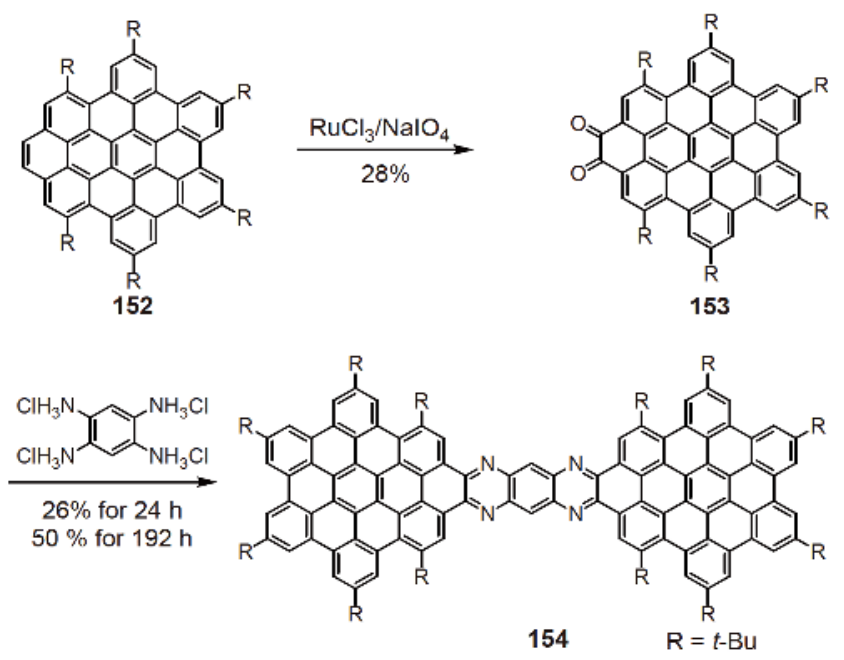

Figure 29 Edge oxidation of $p$-HBC derivative $\mathbf{1 5 2}$ at K-region and further functionalizations.

cohol in liquid ammonia [233]. Hydrogenation of graphene faces difficulties in controlling reaction sites and in elucidating the resulting structure, thus hindering a reliable structure-property correlation. In this regard, hydrogenation of large PAHs is important as a case study on well-defined molecular nanographenes. In 2004, Watson and Müllen et al. [234] reported hydrogenation of $p$-HBC derivatives $\mathbf{1 5 5}$ under moderate hydrogen pressure in the presence of palladium on activated carbon as catalyst (Figure 30). Nuclear magnetic resonance (NMR) experiments strongly indicated an all-syn addition of hydrogen atoms to the peripheral carbons of $p$-HBCs, resulting in stereoselective generation of peralkylated coronenes 156 .

Similarly, the established halogenation protocols of graphene fail to achieve the atomic accuracy, which would be necessary for reliable structure-property correlations $[229,230]$. For example, a non-destructive photochemical chlorination protocol (Figure 31) has been employed by Liu et al. [235] to attach chlorine atoms to the basal carbon of graphene, providing chlorinated graphene with a coverage of approximately 8 atom $\%$ chlorine and an opened band gap of $45 \mathrm{meV}$. Alternatively, a chlorine plasma reaction has been used by Dai et al. [236] to chlorinate graphene non-destructively and reversibly, achieving a coverage of 8.5 atom $\%$ chlorine. Longer exposure times ( $>2 \mathrm{~min}$ ) afforded the irreversible formation of larger-area defects. With these chlorination methods, the band gaps and electronic properties of the resulting graphene derivatives can be effectively tuned, but precise control of the structure remains a major challenge.

To achieve atomic precision, Tan, Feng and Müllen et al. [43] developed an efficient edge chlorination protocol for nanographene molecules with an excess amount of iodine monochloride and a catalytic amount of aluminum chloride 


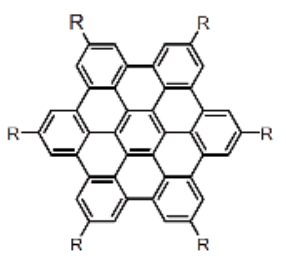

155
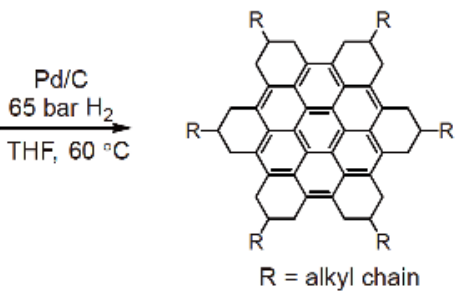

156
Figure 30 Regioselective hydrogenation of $p$-HBC derivatives 155 toward peralkylated coronenes 156.

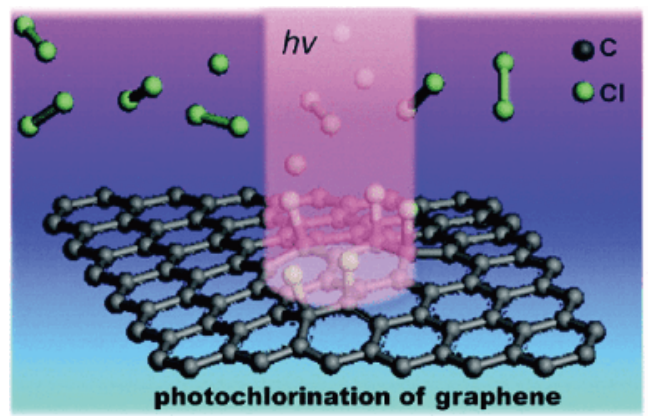

Figure 31 Schematic illustration of photochemical chlorination of graphene. Reprinted with permission from Ref. [235], copyright (2011) American Chemical Society (color online).

(Figure 32). Chlorinated nanographene molecules (157-162) exhibit enhanced solubility in common organic solvents; thus, the single-crystal growth of such large PAHs is possi- ble. Not only the detailed bond lengths of the graphenic structures but also the contorted geometries induced by the steric hindrance between chlorines have been unambiguously elucidated. Another interesting finding is that hydrogens in the gulf regions (marked red in $\mathbf{1 6 0}$ and 161, Figure 32) are not substituted by chlorines in this reaction, probably due to the existing steric hindrance. Furthermore, chlorination leads to decreases in the optical band gaps as well as the highest occupied molecular orbital (HOMO) and lowest unoccupied molecular orbital (HUMO) energy levels.

Apart from the electronic effect upon chlorination, it also provides a new entry to the functionalization and derivatization of nanographene molecules. For instance, Tan et al. [237] demonstrated selective amination at the vertexes of the chlorinated nanographene molecules $\mathbf{1 5 7}$ and $\mathbf{1 5 9}$ through palladium-catalyzed Buchwald-Hartwig $\mathrm{C}-\mathrm{N}$ cross-couplings (Figure 33). The resulting structures 163 and 164 were revealed by X-ray crystallography, representing a new type of donor-acceptor nanographene molecules, of which the optical and assembly properties can be finely modulated by varying the anilino groups.

In addition, starting from perchlorinated $p$-HBC, Tan, Feng and Müllen et al. [238] achieved a peripheral sulfur annulation by a thiolation reaction (Figure 34). Trisulfurannelated $p$-HBC $\mathbf{1 6 5}$ decorated with arylthio groups were obtained in $35 \%-50 \%$ yields, and the disulfur-annelated counterpart 166 was also observed with low yields of $1.5 \%-$ $3 \%$. Compared with the parent $p-\mathrm{HBC}$, the trisulfur-anne-
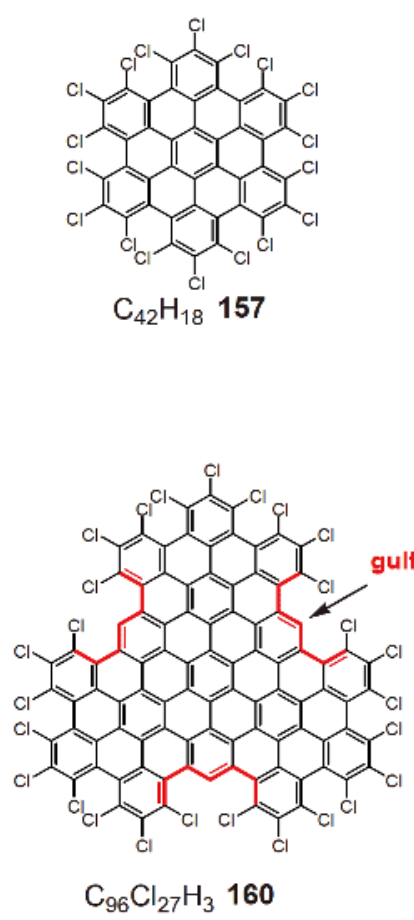

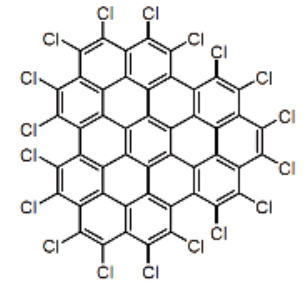

$\mathrm{C}_{48} \mathrm{H}_{18} 158$

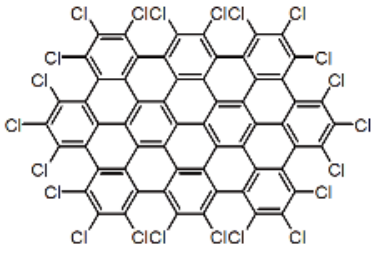

$\mathrm{C}_{60} \mathrm{Cl}_{22} 159$

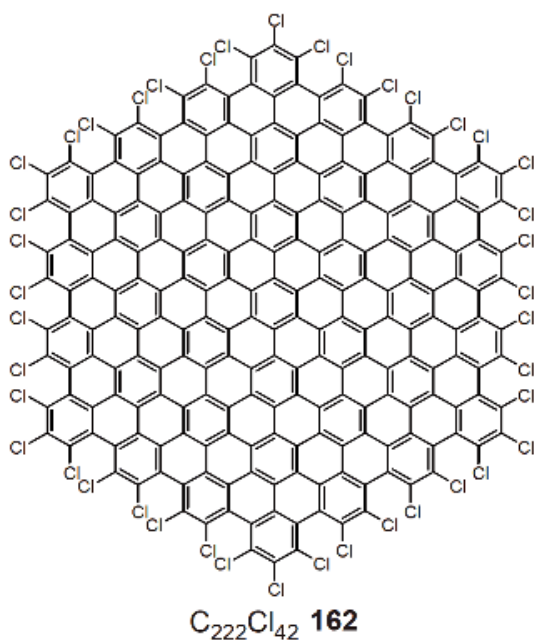

Figure 32 Examples of edge-chlorinated nanographene molecules. The gulf regions as indicated by the red color are not chlorinated due to the steric hindrance (color online). 


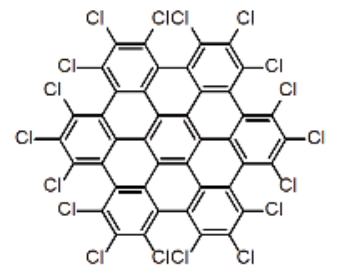

157

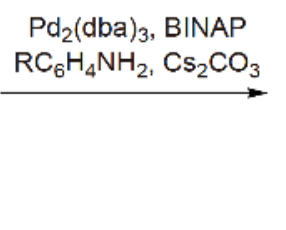

163a: $\mathrm{R}=\mathrm{H}(31 \%), 163 \mathrm{~b}: \mathrm{i}-\operatorname{Pr}(32 \%), 163 \mathrm{c}: n-\mathrm{Bu}(26 \%)$,

163d: $\mathrm{OCH}_{3}(26 \%), 163 \mathrm{e}: \mathrm{N}\left(\mathrm{CH}_{3}\right)_{2}(21 \%)$

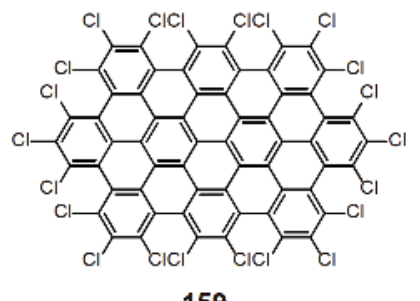

$\mathrm{Pd}_{2}(\mathrm{dba})_{3}$, BINAP

$\underset{\mathrm{RC}_{6} \mathrm{H}_{4} \mathrm{NH}_{2}, \mathrm{Cs}_{2} \mathrm{CO}_{3}}{\longrightarrow}$

$13 \%-20 \%$
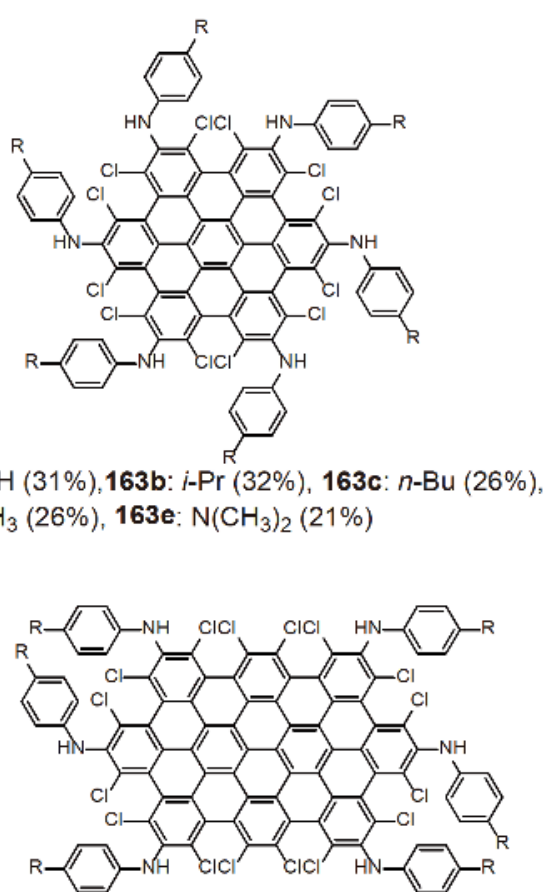

164a: $\mathrm{R}=\mathrm{H}(17 \%)$, 164b: $n-\mathrm{Bu}(17 \%), 164 \mathrm{c}: \mathrm{OCH}_{3}(17 \%)$

Figure 33 Synthesis of donor-acceptor nanographene molecules 163 and 164 through Buchwald-Hartwig C-N cross-couplings of perchlorinated precursors. $\mathrm{Pd}_{2}(\mathrm{dba})_{3}$ : tris(dibenzylideneacetone)dipalladium(0); BINAP: 2,2'-bis(diphenylphosphino)-1,1'-binaphthalene.

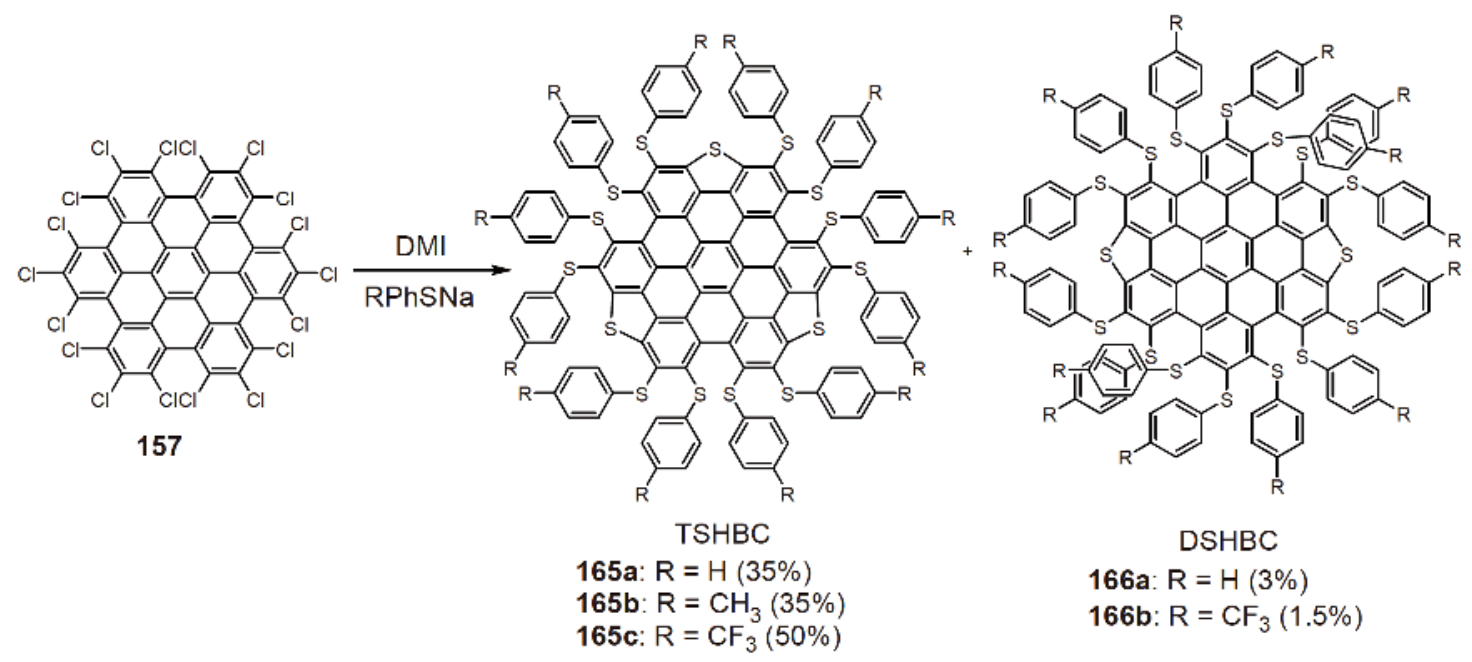

Figure 34 Synthesis of sulfur-annelated nanographene molecules 165 and 166 through thiolation of perchlorinated $p$-HBC 157. DMI: 1,3- dimethyl-2imidazolidinone.

lated $p$-HBC 165 displays a significant red shift of the absorption maximum and a decrease in the optical energy gap of $0.5 \mathrm{eV}$. Remarkably, the thiolated trisulfur-annelated $p$ HBC 165a has been employed as hole transporting material (HTM) in perovskite solar cells, yielding the best efficiency of $12.8 \%$ and an improved efficiency of $14.0 \%$ by further involving graphene sheets in the hole transporting layer [239]. The devices that use 165a as the HTM have shown improved stability compared with those employing the wellestablished HTM, 2,2',7,7'-tetrakis[N,N-di(4-methox- yphenyl)amino]-9,9'-spirobifluorene (Spiro-OMeTAD), presumably due to the hydrophobic nature of the thiolated nanographene molecule. Furthermore, nanographene molecule 165a has been used as a precursor in the bottom-up fabrication of continuous, uniform, and ultrathin sulfurdoped graphene films for ultrahigh-rate micro-supercapacitors [240].

The same perchlorination protocol was applied to coronene by Müllen and Feng et al. [241] (Figure 35). Nucleophilic substitution of all peripheral chlorines with 

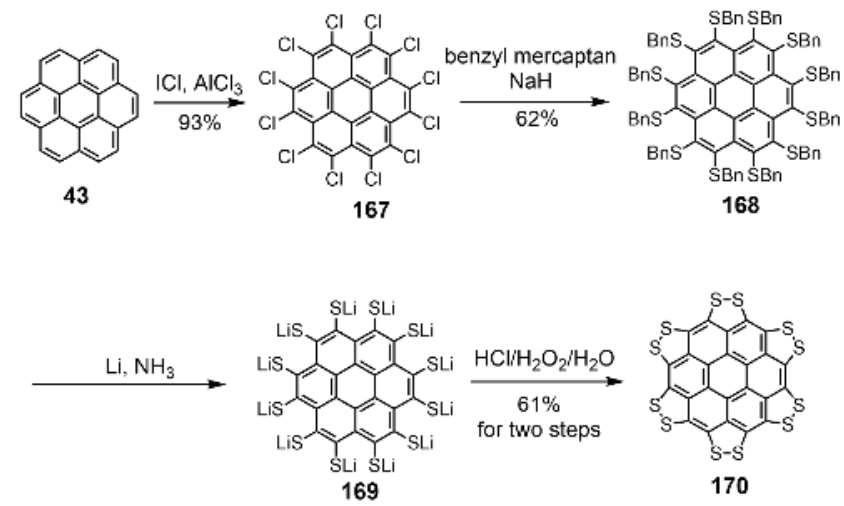

Figure 35 Synthesis of persulfurated coronene 170.

lithium benzylthiolate and subsequent reductive cleavage of the benzyl groups under Birch conditions afford intermediate 169, which is treated with aqueous hydrogen chloride and hydrogen peroxide to yield the remarkable persulfurated coronene 170. Due to its sulfur-rich character, the persulfurated coronene $\mathbf{1 7 0}$ functions as a promising cathode material for lithium-sulfur batteries, exhibiting a high capacity of $520 \mathrm{~mA} \mathrm{~h} \mathrm{~g}^{-1}$ after 120 cycles and retaining $90 \%$ Coulombic efficiency at $0.6 \mathrm{C}$.

These studies on the edge chlorination and further derivatizations of nanographene molecules have provided clear information on similar GNR modifications. Feng and Müllen et al. [43] conducted the efficient edge chlorination of a tertbutyl-substituted GNR 171 with iodine monochloride and aluminum chloride (Figure 36(a)). The tert-butyl groups were readily substituted by chlorine atoms based on a model reaction of hexa-tert-butyl-hexa-peri-hexabenzocoronene and characterizations by X-ray photoelectron spectroscopy (XPS) as well as infrared spectroscopy (IR). Benefiting from the unambiguous structural analysis of PAHs $\mathbf{1 6 0}$ and $\mathbf{1 6 1}$ bearing gulf regions, especially from the single-crystal structure of PAH 160, one could safely conclude that the hydrogens in the gulf regions of the GNR were unaffected by the chlorination reaction, as further confirmed by XPS and IR analyses. Compared with the pristine GNR 171, the chlorinated GNR 172 displayed a red-shifted absorption maximum (Figure 36(b)) and a decreased band gap by $0.2 \mathrm{eV}$. The edge chlorination is effective to modulate the optical and electronic properties of GNRs without changing the backbone. Nevertheless, further derivatizations as conducted on the aforementioned chlorinated PAHs are challenging for the chlorinated GNRs because highly efficient reactions are needed to guarantee a high degree of clean transformations.

To achieve further edge functionalizations of GNRs, Müllen and Narita et al. [242] first synthesized a polyphenylene precursor 174 bearing bromo groups through an AB-type Diels-Alder polymerization. The functional moieties were then attached to the polyphenylene backbone by a highly efficient Suzuki coupling before the final cyclodehydrogenation (Figure 37(a)). In 2017, three electron-deficient units, i.e., anthraquinone (AQ), naphthalene monoimide (NMI) and perylene monoimide (PMI), were attached to the edge of the GNRs to lower the band gap as well as the conduction and valence band energy levels. Interestingly, AFM studies on the films of these GNRs on graphite revealed that different substituents at the edges affected the self-assembly properties of the GNRs. Whereas the AQ- and NMI-functionalized GNRs exhibited domains of closely packed GNRs, the PMI-functionalized GNRs provided unique rectangular networks with single strands or bundles of GNRs arranged in a perpendicular way. This perpendicular "crossing" of the GNRs was likely due to the strong interaction between the GNR backbone and the pendant PMI moieties. In this regard, an early work on the selfassembly of $\mathrm{HBC} /$ perylene diimide dyads could help to understand the peculiar behavior of GNRs [243]. Remarkably, through the bromo-functionalized GNR, which was synthesized by cyclodehydrogenation of the same polyphenylene percursor bearing bromo groups as described above, nitronyl-nitroxide (NIT) radicals were introduced at the edges of the GNRs (177d) [244]. Spin injection from the NIT radicals into the GNR backbone was revealed by electron spin resonance spectroscopy in conjunction with spin density calculations, demonstrating the existence of magnetic edge states of such radical-functionalized GNRs. Different from
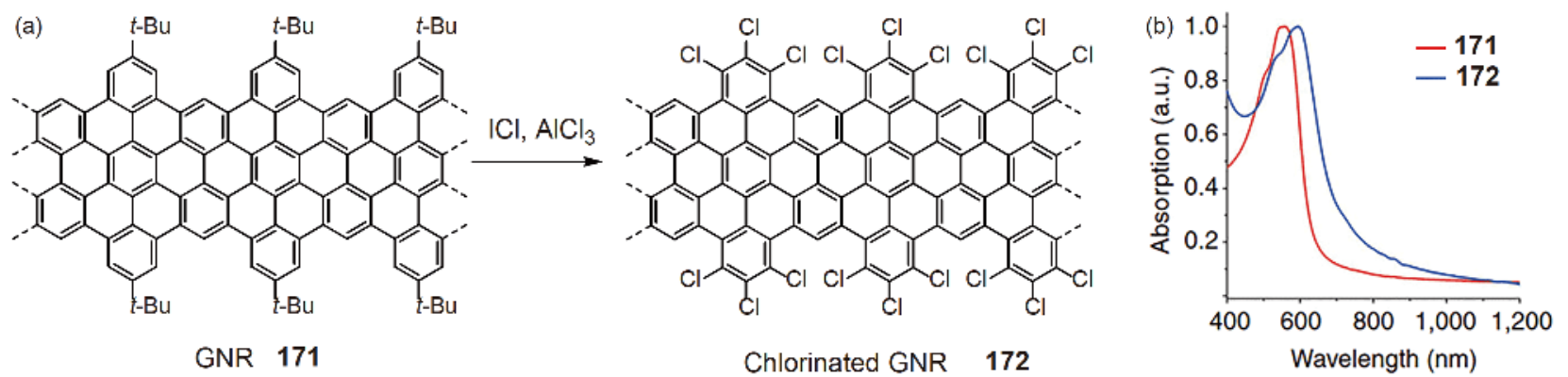

Figure 36 (a) Edge chlorination of GNR 171 to chlorinated GNR 172; (b) UV-Vis spectra of GNR 171 and chlorinated GNR 172. Reprinted with permission from Ref. [43], copyright (2013) Macmillan Publishers Ltd. (color online). 

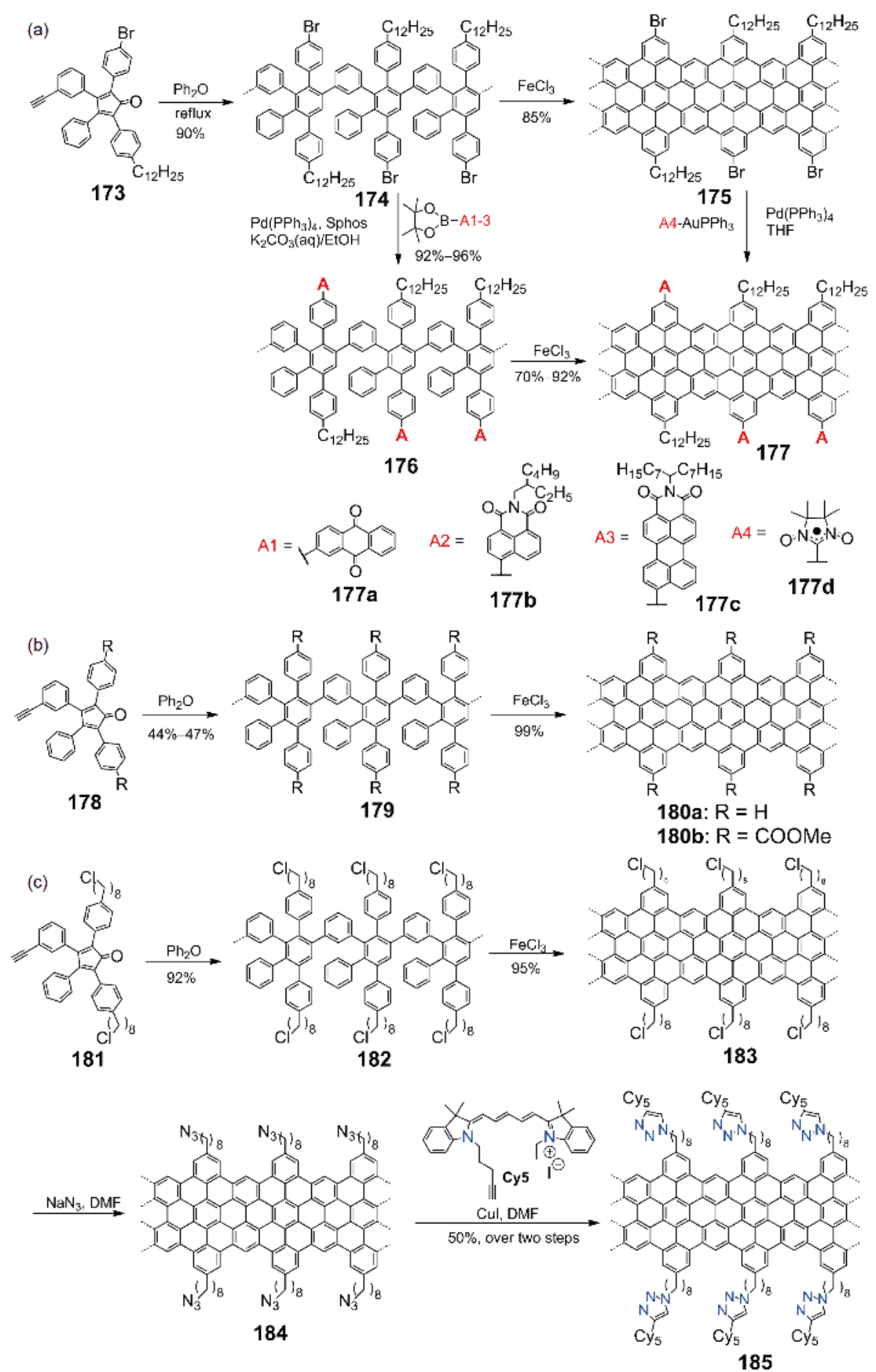

Figure 37 Edge functionalization of GNRs with different functional groups. (a) Synthesis of GNR 177 bearing electron-deficient and radical units; (b) synthesis of GNR 180 with methyl ester groups; (c) synthesis of GNR 185 with fluorescent dyes along the edge. $\operatorname{Pd}\left(\mathrm{PPh}_{3}\right)_{4}$ : tetrakis(triphenylphosphine) palladium(0); Sphos: 2-dicyclohexylphosphino-2',6'-dimethoxybiphenyl; DMF: dimehylformamide (color online).

the unstable ZGNRs, which were only obtained and characterized under UHV [173], the NIT-radical-functionalized GNRs were stable under ambient conditions and could be processed in the liquid phase, thereby providing a promising alternative for the spintronic applications of GNRs [245]. Employing this concept of modifying the GNR edges for different functions, Fischer and Xu et al. [246] synthesized the same GNR structure with methyl esters (-COOMe) on the edges 180b (Figure 37(b)). Composite electrodes comprising gold nanoparticles and the synthesized GNRs displayed a synergistic performance enhancement for the electrocatalytic reduction of $\mathrm{CO}_{2}$. Thereafter, Fischer and $\mathrm{Xu}$ 
et al. [247] reported GNR 185 decorated with fluorescent dyes along the edges for super-resolution imaging. The synthesis relied on a key GNR 184 featuring pendant azide groups, which was used to attach the dye molecules by the copper-catalyzed azide-alkyne cycloaddition (CuAAC click reaction) (Figure 37(c)).

The regiospecific edge functionalization of nanographene molecules and GNRs has provided reliable structure-property correlations and facilitated further derivatizations for a variety of applications. However, the functionalization of graphene, either at the edge or on the basal plane, has often been performed in a less controlled way. Such ill-defined functionalized graphene materials are undoubtedly of technological value, but an ideal site-specific functionalization would be important to reveal the intrinsic physical properties. For example, atomic-scale control of graphene hydrogenation allowed for unambiguous characterization of the induced magnetic moment and the spatial extension of the spin-polarized electronic state by STM [248]. Furthermore, by using the STM tip to manipulate chemisorbed hydrogen atoms, the magnetism of certain graphene regions could be accurately tailored (Figure 38). Such advances are still far from real-world applications, but the atomic precision, as observed in the molecular nanographenes, is essential to the fundamental understanding and use of graphene functionalizations.

\section{3 "Defects" make the difference}

Perfect graphene consists of $\mathrm{sp}^{2}$-hybridized carbons arranged in the 2D hexagonal lattice, but in reality a variety of structural defects are unavoidably generated during the fabrication of graphene and greatly affect its mechanical, thermal, electronic, and magnetic properties [249-253]. The defects can also be intentionally introduced into graphene by ion or electron irradiation to modulate its properties [250]. The simplest type of defect is based on vacancy, which is known as Schottky defects and formed by removing carbon atoms from the graphene lattice [254]. The vacancy defects (Figure 39) typically include single vacancy (one missing carbon), double vacancy (two missing carbons), and multivacancy (multiple missing atoms) [250]. Another deviation from the ideal structure is known as Stone-Wales (or StoneThrower-Wales) defects $[255,256]$, which are created by reconstruction of the graphene lattice without removing or adding additional carbon atoms. When one of the $\mathrm{C}-\mathrm{C}$ bonds is rotated by $90^{\circ}$, four adjacent hexagons are transformed into an isomeric form with a pair of joined heptagons placed between a pair of pentagons [250]. Besides the abovementioned point defects, line defects are often observed at the grain boundaries of polycrystalline graphene [257-259]. Such line defects are predominantly composed of non-hexagonal rings, e.g., pentagons, heptagons, and octagons,

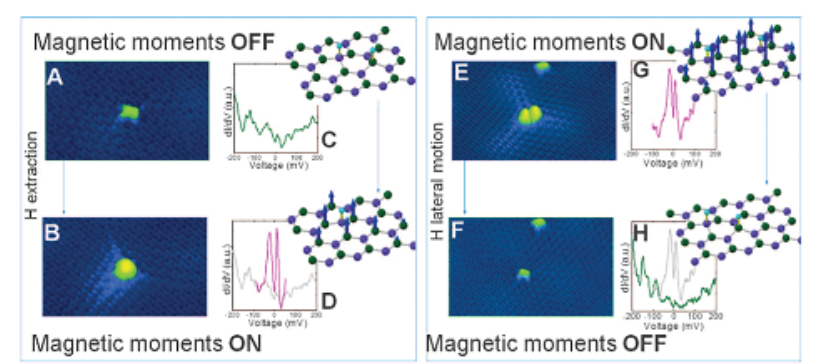

Figure 38 Manipulation of hydrogen atoms on graphene by STM to modulate local magnetic moments. Reprinted with permission from Ref. [248], copyright (2016) American Association for the Advancement of Science (color online).
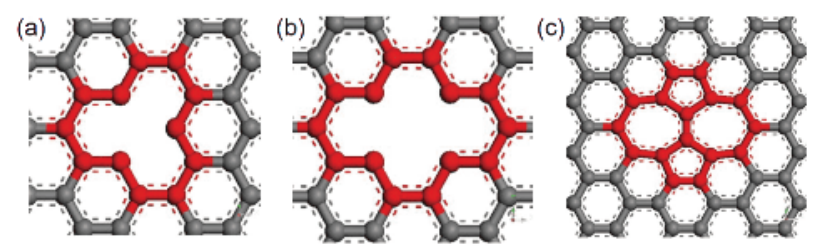

Figure 39 Schematic illustration of different defects in graphene sheets: (a) single vacancy, (b) double vacancy, and (c) Stone-Wales defect. Reprinted with permission from Ref. [252], copyright (2012) Royal Society of Chemistry (color online).

having pronounced effects on the electronic transport of graphene [260].

When a vacancy is generated, the lattice may undergo reconstructions by relaxing into a lower energy state [250]. A Jahn-Teller distortion occurs at the single vacancy, leading to covalently bonding between two of the three dangling bonds and leaving one dangling bond for geometrical reasons. Therefore, the single vacancy leads to quasi-localized states near the Fermi level, endowing graphene with magnetic properties [261-263]. The reconstruction of such defects results in a new configuration with a five-membered and nine-membered ring (5-9 defect, Figure 40(a)). Similarly, a reconstructed double vacancy generates two pentagons and one octagon (5-8-5 defect, Figure 40(b)). Such double-vacancy defects have no dangling bonds; thus, they are thermodynamically more favored than the single vacancy. The 5-8-5 defect is not the only reconstruction mode in the case of two missing carbon atoms. Similar to the generation of a Stone-Wales defect, the rotation of a carbon-carbon bond (marked by an arrow in Figure 40(b)) in the octagon of the 58-5 defect creates a pattern of three pentagons and three heptagons (555-777 defect, Figure 40(c)). Further rotation of another bond (marked by an arrow in Figure 40(c)) in the 555-777 defect leads to a new 5555-6-7777 configuration (Figure 40(d)). Indeed, these reconstructed defects have experimentally been identified by high-resolution transmission electron microscopy [264]. If a large number of carbon atoms are removed from the graphene lattice, then numerous 
(a)
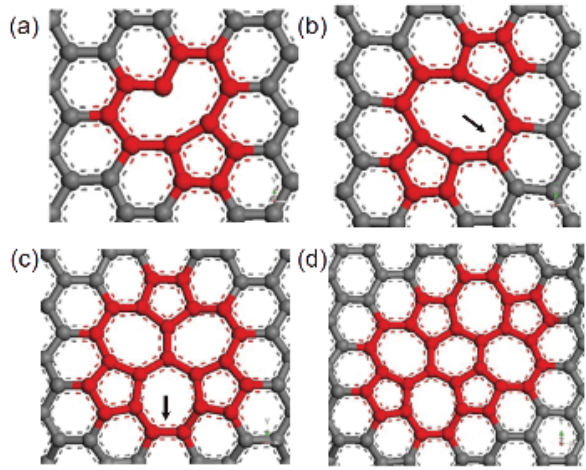

Figure 40 Schematic illustration of reconstructed single- and doublevacancy defects in graphene sheets: (a) single vacancy, (b) double vacancy (5-8-5), (c) double vacancy (555-777), and (d) double vacancy (5555-67777). Reprinted with permission from Ref. [252], copyright (2012) Royal Society of Chemistry (color online).

complex defect configurations would be obtained, forming either a random arrangement of non-hexagonal polygons or hole defects $[265,266]$.

During the generation of a vacancy, the leaving atom may stay on the surface of graphene, forming an adatom-vacancy pair, which is an analog to a Frenkel defect [267]. The adatoms are relatively mobile on graphene, so a carboncarbon dimer can be generated when two migrating adatoms meet each other. The dimer can be incorporated into the graphene network, leading to a specific defect composed of two joined pentagons placed between two heptagons. Such an arrangement results in a local curvature and is termed inverse Stone-Wales defect (Figure 41(a)) $[268,269]$. While the graphene defects relating to pentagon-heptagon pairs seem to have different patterns (Figure 41(b)), organic chemists would be aware of naphthalene $\mathbf{1 2 2}$ and its isomer azulene 186. Furthermore, pyrene 88a and its three nonbenzenoid isomers, i.e., dicyclopenta[ef,kl]heptalene $\mathbf{1 8 7}$ [270], dicyclohepta[cd,gh]pentalene 188 [271], and acepleiadylene 189 [272] have been known for many decades. Notably, molecules 187 and $\mathbf{1 8 8}$ display the pentagon-heptagon arrangement of the Stone-Wales defect and the inverse Stone-Wales defect, respectively; however, the other pyrene isomer, acepleiadylene 189, has not been given a defect term by physicists. Indeed, the characterization and structural control of graphene defects are still challenging in the graphene field. The development of PAH chemistry, particularly, of PAHs with non-six-membered rings and other "defect" features, can be considered as useful model compounds for defective graphene because the geometrical and electronic consequences of the "defects" in PAHs can be clearly evaluated.

A typical PAH having an embedded five-membered ring is corannulene 17, which is a bowl-shaped fullerene (C60) fragment and has a dipole moment of 2.1 Debye due to the different electron densities on its concave and convex sur-
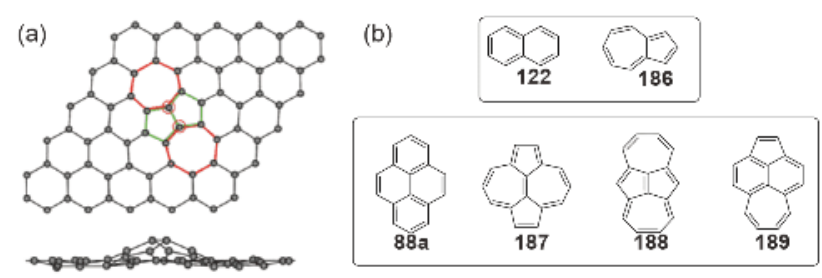

Figure 41 (a) DFT-optimized structural model of inverse Stone-Wales defect formed by incorporating a pair of adatoms into the graphene lattice. Reprinted with permission from Ref. [250], copyright (2011) American Chemical Society. (b) Several small PAHs that are relevant to the graphene defects, including naphthalene and its isomer azulene 186, pyrene, and its three isomers: dicyclopenta[ef,kl]heptalene 187, dicyclohepta $[c d, g h]$ pentalene 188, and acepleiadylene $\mathbf{1 8 9}$ (color online).

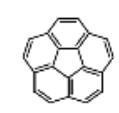

17

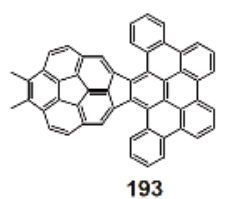

193

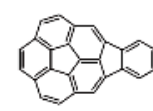

190

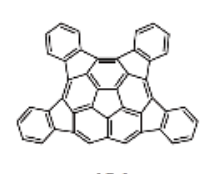

194

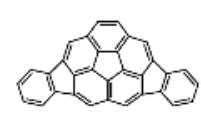

191

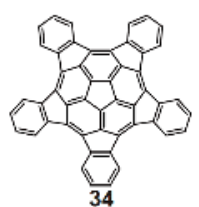

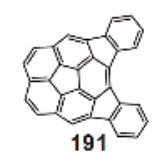

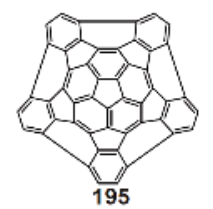

Figure 42 Examples of $\pi$-extended corannulene derivatives.

faces. A number of $\pi$-extended corannulene derivatives (Figure 42) have been synthesized with different bowl depths and solid-state packing modes. These compounds are of particular interest by their own value in supramolecular selfassembly, energy storage, and organic electronics [273]. Such corannulene-based PAHs are substructures of graphene with pentagon defects, which result in local positive (spherical) curvature. Recently, Zhang and $\mathrm{Mu}$ et al. [274] revealed that the intrinsic pentagon defects in graphene materials resulted in a local charge redistribution, providing superior binding affinity toward oxygen. The pentagon-rich graphene materials exhibited high electrochemical reactivity in the oxygen reduction reaction (ORR) for fuel cells and $\mathrm{Zn}$ air batteries, as well as high specific capacitance in electric double-layer supercapacitors. Such a pronounced effect of pentagon defects in graphene materials can be interpreted by comparing the model compounds, such as the all-hexagon coronene 43 and pentagon-embedded corannulene (17). Theoretical studies indicated that high charge densities are localized on the five-membered ring of the corannulene motif, thus resulting in high oxygen adsorption energies [274].

The chemistry of corannulene has also stimulated the development of other types of PAHs embedding five-membered rings. For example, Feng and Müllen et al. [275] described a multi-step synthesis of a fragment of C70 fullerene and other higher fullerenes (198, Figure 43(a)). The 


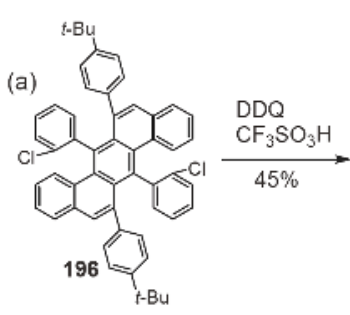

(b)

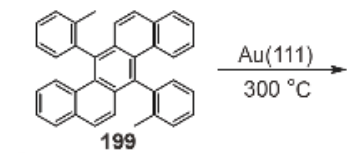

(d)

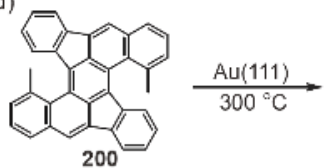

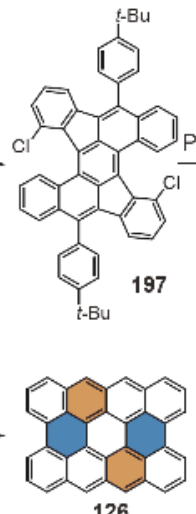

126

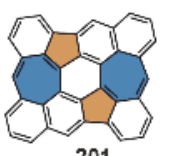

201

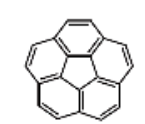

Coranulene 17

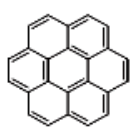

Coronene 43

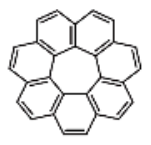

[7]Circulene 202

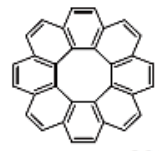

[8]Circulene 203
Figure 44 Examples of $[n]$ circulenes containing five-, six-, seven- or eight-membered rings.

graphene [250]. An important class of such compounds includes [7]circulene 202 and [8]circulene 203, as depicted in Figure 44. By definition, PAHs with an $n$-membered ring as a core, which is fully surrounded by fused benzene rings, are $[n]$ circulenes. Accordingly, corannulene $\mathbf{1 7}$ and coronene 43 can be regarded as [5]circulene and [6]circulene, respectively. [7]Circulene was initially synthesized by Yamamoto and Nakazaki et al. in 1983 [277], and the synthesis of [7.7]circulene, which had two seven-membered rings, was reported in 1991 [278]. Other than circulenes, limited examples of nanographene molecules with embedded sevenmembered rings are available.

In 2012, a $p$-HBC analog 205 with an extra $\mathrm{sp}^{3}$-carbon was reported by Miao et al. [209] as a new nanographene molecule incorporating a seven-membered ring (Figure 45(a)). The synthesis was based on the oxidative cyclodehydrogenation of hexaphenylbenzene-like precursor 204, in which the introduction of alkoxyl groups at ortho or para positions with regard to the reaction sites was found important to achieve complete cyclodehydrogenation. A nonplanar geometry was observed for PAH $\mathbf{2 0 5}$ as evidenced by single-crystal X-ray analysis. Then, in 2015, new saddleshaped nanographene molecules $\mathbf{2 0 8}$ and 210 incorporating two heptagons were synthesized by the same group (Figure 45(b)) [279]. The saddle-shaped diketones 206 having two tropone subunits were used as the key precursors for the synthesis. Such saddle-shaped structures feature a negative Gaussian curvature. In 2018, Miao et al. [280] reported another type of negatively curved nanographene molecules 214 involving tetrabenzodipleiadiene as a building block (Figure 45(c)). The single-crystal structure revealed the saddleshaped backbone with a highly distorted naphthalene moiety in the center. Recently, Martín et al. [281] reported the synthesis of curved nanographene molecules from corannulene (Figure 46). The Scholl reaction in the final step led to different products depending on the oxidation conditions. With $\mathrm{FeCl}_{3}$ at a low temperature, a helical structure $\mathbf{2 1 6}$ containing a [6] helicene moiety was generated, whereas with 2,3-dichloro-5,6-dicyano-1,4-benzoquinone and trifluoromethanesulfonic acid, an additional $\mathrm{C}-\mathrm{C}$ bond was formed, leading to a new seven-membered ring. The resulting non-planar nanographene molecule 217 features both positive curvature from corannulene and negative curvature from the embedded heptagon.

Whereas [7]circulene was first synthesized in 1983, the

Compared with five-membered rings, seven- and eight- 
(a)

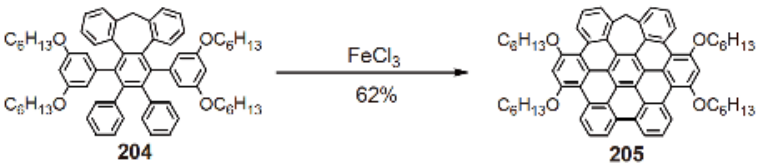

(b)
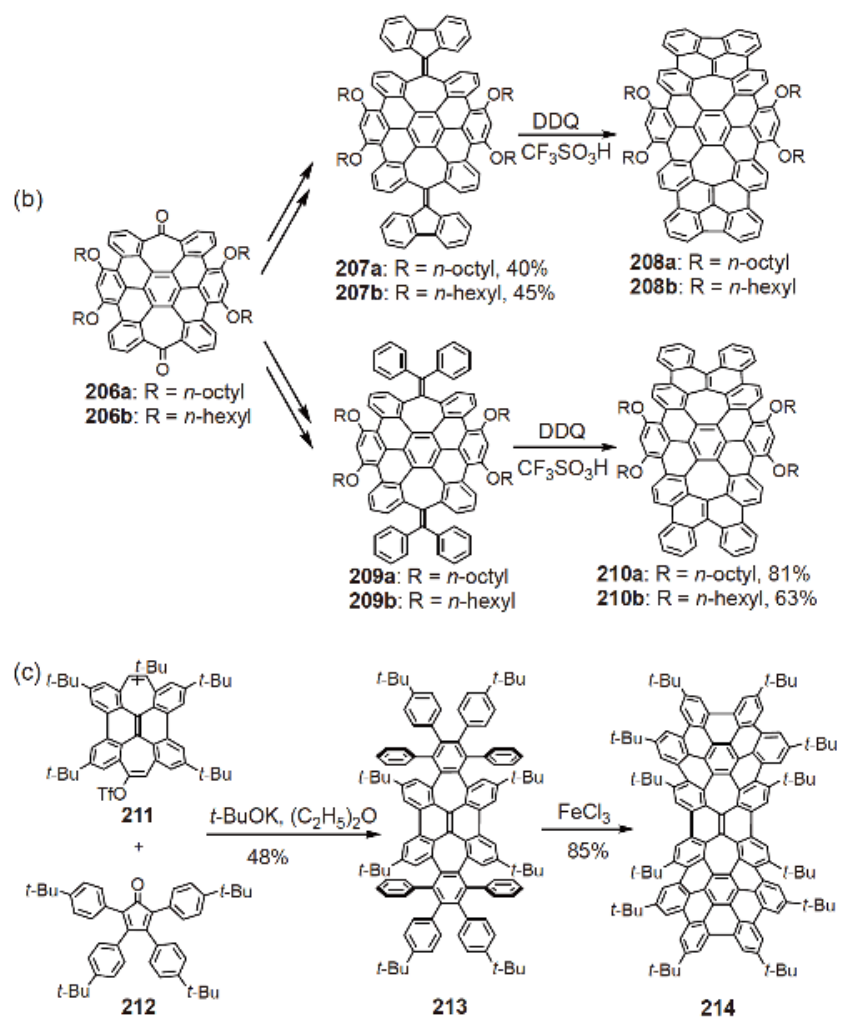

Figure 45 (a) Synthesis of PAH 205 with an extra $\mathrm{sp}^{3}$-carbon; (b) synthesis of curved nanographene molecules 208 and $\mathbf{2 1 0}$ with embedded seven-membered rings; (c) synthesis of negatively curved nanographene molecules 214

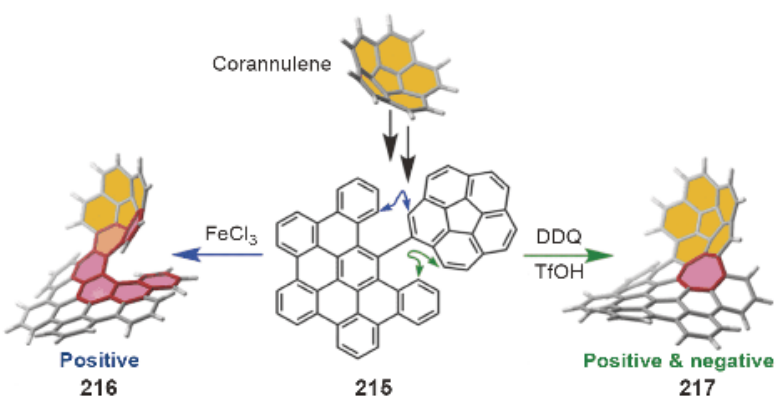

Figure 46 Synthesis of corannulene-based nanographene molecules 216 with positive curvature and $\mathbf{2 1 7}$ with both positive and negative curvature. Reprinted with permission from Ref. [281], copyright (2018) American Chemical Society (color online).

synthesis of [8]circulene was only achieved three decades later. In 2013, Wu et al. [282] reported the successful synthesis of peri-substituted [8]circulene 219 through Pdcatalyzed annulations of tetraiodo-substituted tetraphenylene 218 with diarylethynes (Figure 47(a)). Later, tetrabenzo[8] circulene $\mathbf{2 2 4}$ was independently synthesized by two groups through different strategies (Figure 47(b)). Sakamoto and Suzuki et al. [283] adopted a method employing oxidative

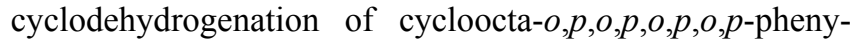
lene 220, whereas Whalley et al. [284] developed another protocol through a sequence of Diels-Alder cycloaddition and Pd-catalyzed intramolecular cyclization. Single-crystal X-ray analysis of tetrabenzo[8]circulene $\mathbf{2 2 4}$ revealed a deeply saddle-shaped geometry with two $S_{4}$-symmetric conformers. An even more $\pi$-extended nanographene molecule $\mathbf{2 2 7}$ employing [8]circulene as the core structure (Figure 47(c)) was synthesized by Miao et al. [285] in 2017. The key steps rely on a Diels-Alder reaction of a macrocyclic diyne 225 and the following Scholl reaction. The resulting large PAH displayed a twisted conformation instead of the saddleshaped conformation as shown in other [8]circulene derivatives.

Although the synthesis of the aforementioned PAHs with non-six-membered rings has been mainly motivated by the fundamental interest of organic chemists, the variety of such nanographene molecules have indeed provided a number of molecular models for defective graphene. As the geometrical effect of non-hexagonal defects in the graphene lattice, such as the predicted non-planarity in the inverse Stone-Wales defect (Figure 41(a)), cannot be easily investigated by STM or TEM, such local structural features could be elucidated by analyzing the corresponding PAHs, especially through the powerful X-ray crystallography. Furthermore, the higher activity of the defective graphene in the electrocatalytic process with regard to the pristine graphene requires a "clear picture" of the active sites [286,287]. These PAH structures with various non-hexagonal "defects" could facilitate the identification of active structures and further promote the rational design of metal-free graphene materials for electrocatalysis.

The PAHs embedding non-hexagonal rings have been largely exploited in recent years, but incorporating such ring defects into GNRs is a challenging task. In the molecular systems, the non-six-membered rings often result in nonplanar geometries and low efficiencies of the ring-closure step. These factors would make the process of synthesizing and characterizing GNRs with such structural features less straightforward. In 2017, Qiu and Zhong et al. [288] reported the on-surface synthesis of a new type of GNR 231 with regularly embedded four- and eight-membered rings from a 1,6,7,12-tetrabromo-3,4,9,10-perylene-tetracarboxylic-dianhydride monomer 228 (Figure 48). The structure was characterized by high-resolution STM and nc-AFM, revealing a planar geometry. DFT calculations indicated that the highest occupied and lowest unoccupied states were predominantly distributed around the eight- and four-membered rings, respectively, demonstrating a new way to modulate the electronic properties of GNRs.

In addition to the non-six-membered rings, another type of 

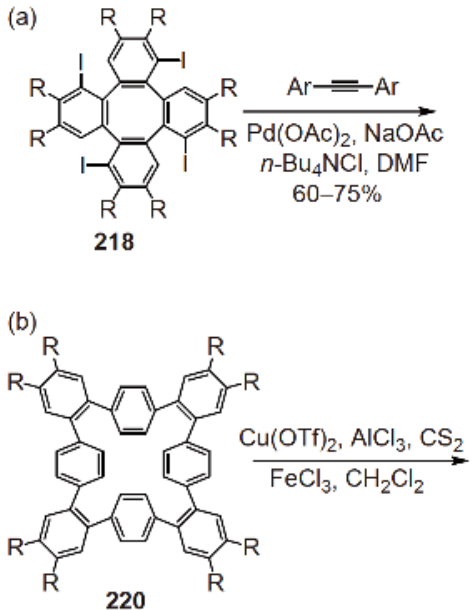

220

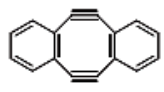

221

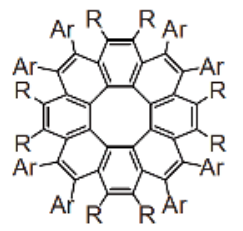

219a: $\mathrm{R}=\mathrm{Me}, \mathrm{Ar}=4-\mathrm{MeC}_{6} \mathrm{H}_{4}$ 219b: $\mathrm{R}=\mathrm{OMe}, \mathrm{Ar}=3,5-\mathrm{Me}_{2} \mathrm{C}_{6} \mathrm{H}_{3}$ 219c: $\mathrm{R}=\mathrm{OMe}, \mathrm{Ar}=4-\mathrm{MeC}_{6} \mathrm{H}_{4}$

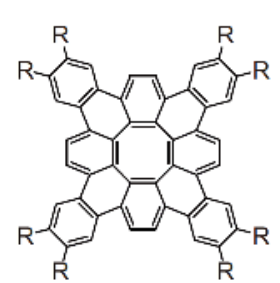
224a: $\mathrm{R}=\mathrm{H}, 7 \%$

224b: $R=M e, 35 \%$ $\operatorname{Pd}\left(\mathrm{PCy}_{3}\right)_{2} \mathrm{Cl}_{2} \underset{\begin{array}{l}\mathrm{DBU}, \mathrm{DMA} \\ \text { microwave } \\ 24 \%\end{array}}{\mathrm{cl} \%}$

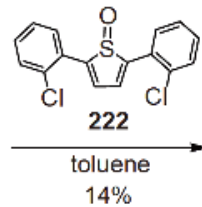
$14 \%$

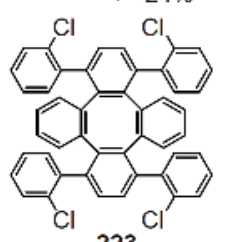

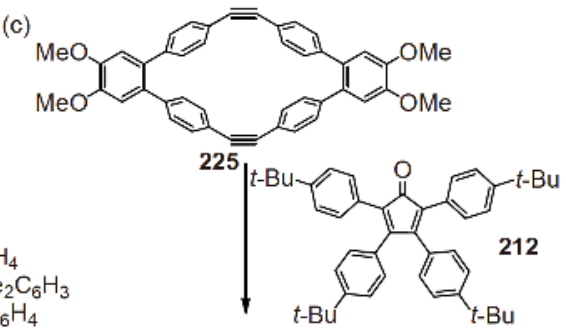

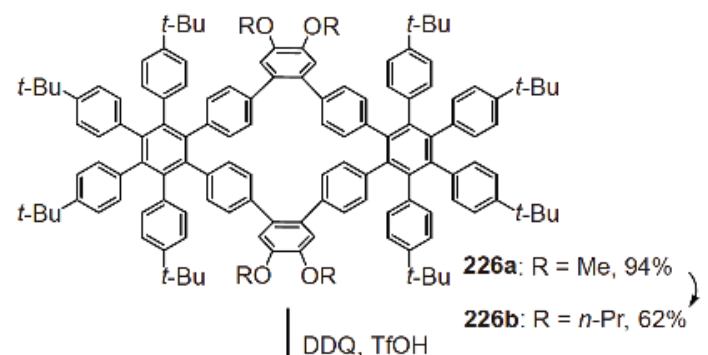

$\mathrm{DDQ}, \mathrm{TfOH}$

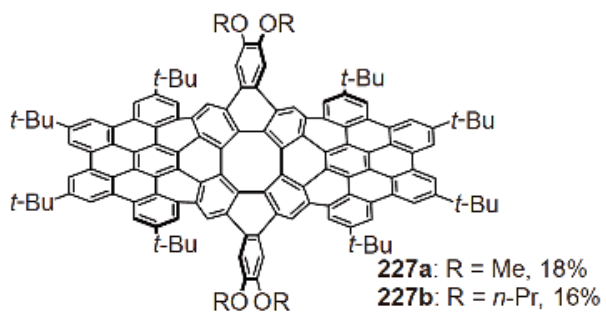

Figure 47 (a) Synthesis of peri-substituted [8]circulene 219; (b) two different synthetic routes to tetrabenzo[8]circulene 224; (c) synthesis of a twisted PAH 227 with an embedded eight-membered ring. Pd(OAc $)_{2}$ : palladium(II) acetate; $\mathrm{Cu}(\mathrm{OTf})_{2}$ : copper(II) trifluoromethanesulfonate; DBU: 1,8-diazabicyclo[5.4.0] undec-7-ene; DMA: dimethylacetamide; DDQ: 2,3-dichloro-5,6-dicyano-1,4-benzoquinone.

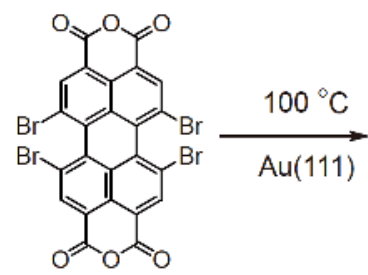

228

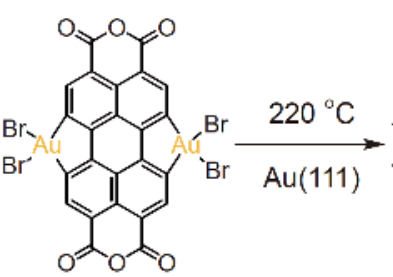

229

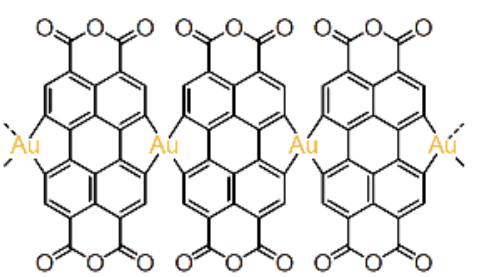

230

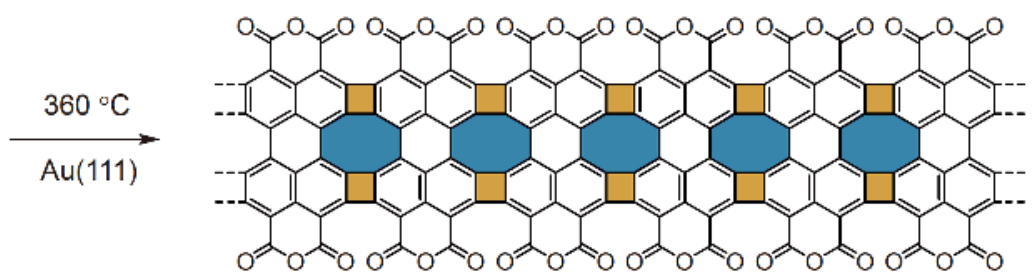

231

Figure 48 Surface-assisted synthesis of GNR 231 with regularly embedded four- and eight-membered rings (color online).

defect is the absence of carbons in the hexagonal lattice, generating hole defects as shown in holey graphene $[289,290]$. Unlike the semimetallic "perfect" graphene, holey graphene with nanopores is predicted to be a semiconductor, which is more favorable for switching electronic devices such as FETs [291,292]. However, as in the case of GNRs, the top-down approach is unable to deliver precise control over the structures in terms of pore size, density, and morphology; the bottom-up synthesis of such graphenic structures with hole defects becomes essential. In 2016, Müllen et al. [293] described the synthesis of a nanographene molecule with $216 \mathrm{sp}^{2}$-carbons (PAH 233, termed C216), which is formally derived from PAH 16 with $222 \mathrm{sp}^{2}-$ carbons (termed $\mathrm{C} 222$ ) by cutting a hole in the middle 

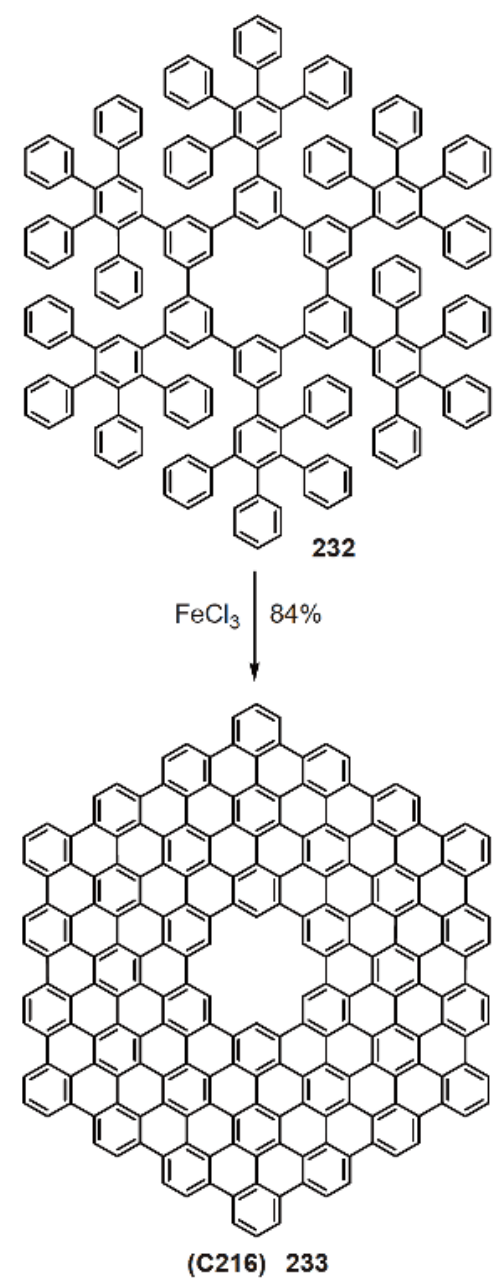

Figure 49 Synthesis of nanographene molecule 233 with a hole defect.

(Figure 49). Notably, FTIR analysis of the holey C216 revealed a "hole marker" originating from a fingerprint peak from $\mathrm{C}-\mathrm{H}$ bonds inside the cavity. Compared with the "perfect" C222, the holey C216 exhibited a decreased HOMO level and an increased LUMO level, thereby resulting in a band gap enlarged by $0.4 \mathrm{eV}$. Although this molecular case implies an effective band gap tuning by introducing hole "defects," further extending the nanographene molecule in the 2D manner toward holey graphene is demanding and requires careful monomer design.

In 2009, Bieri and Fasel et al. [294] together with the group of Müllen took the first step toward porous graphene 235 via the bottom-up procedure (Figure 50). An ordered 2D polyphenylene network with atomic precision was achieved based on the surface-assisted Ullmann-type coupling of a carefully designed precursor, hexaiodo-cyclohexa- $m$-phenylene 234. A uniform pore spacing of $7.4 \AA$ was clearly revealed by STM characterization. In 2018, Moreno, Peña and Mugarza et al. [295] developed another strategy and achieved the bottom-up synthesis of nanoporous graphene by taking advantage of the on-surface synthesis protocol of
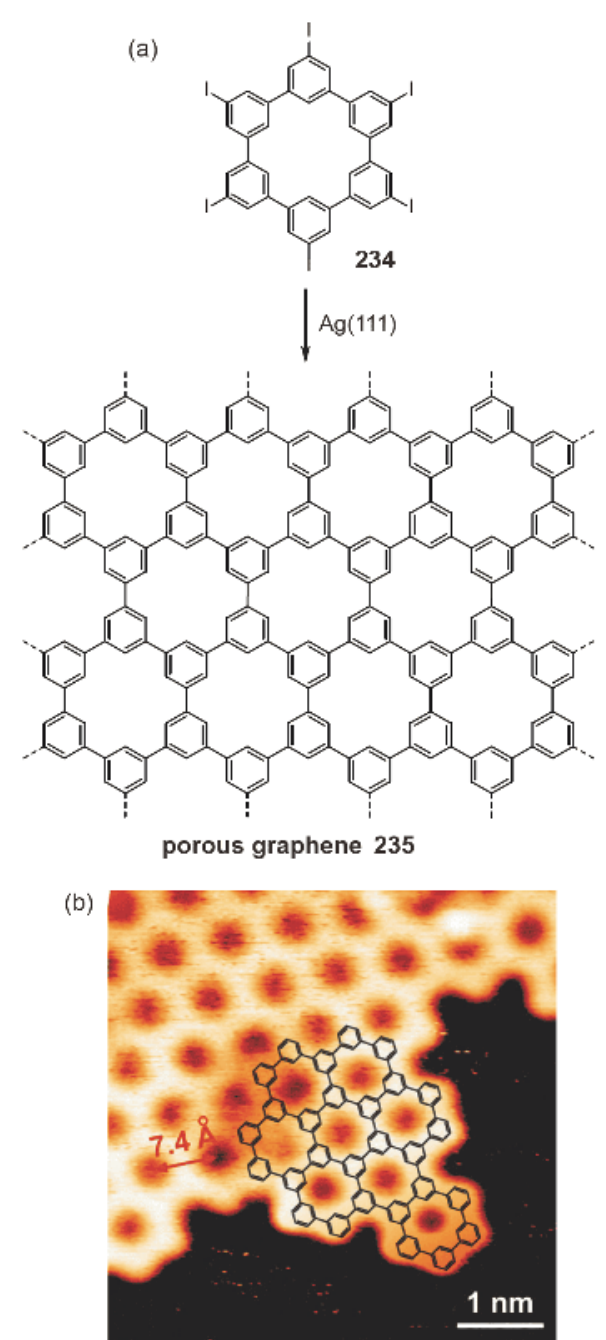

Figure 50 (a) Surface-assisted synthesis of 2D polyphenylene networks; (b) STM image of an edge of the polymer network. (b) Reprinted with permission from Ref. [294], copyright (2009) Royal Society of Chemistry (color online).

GNRs (Figure 51). They used 2,2'-diphenyl-10,10'-dibromo9,9'-bianthracene $\mathbf{2 3 6}$ as the monomer and first synthesized the edge-extended 7-AGNRs 238, as in most of the GNR syntheses. Subsequent dehydrogenative lateral coupling of the GNRs at a higher temperature led to the covalently interconnected GNR array, which is, in another view, nanoporous graphene 239. Althougth the lateral fusion of AGNRs to form wider GNRs has been observed [164,170,296,297], the additional phenyl ring in the bianthracene monomer is the key to the formation of the nanoporous graphene.

\subsection{Heteroatom doping}

Doping of silicon-based semiconductors by group III (pdoping) or group V (n-doping) elements has played a pivotal role in the semiconductor industry. Similarly, incorporating heteroatoms, such as boron (B) and nitrogen $(\mathrm{N})$, into graphene provides additional opportunities in modulating its 


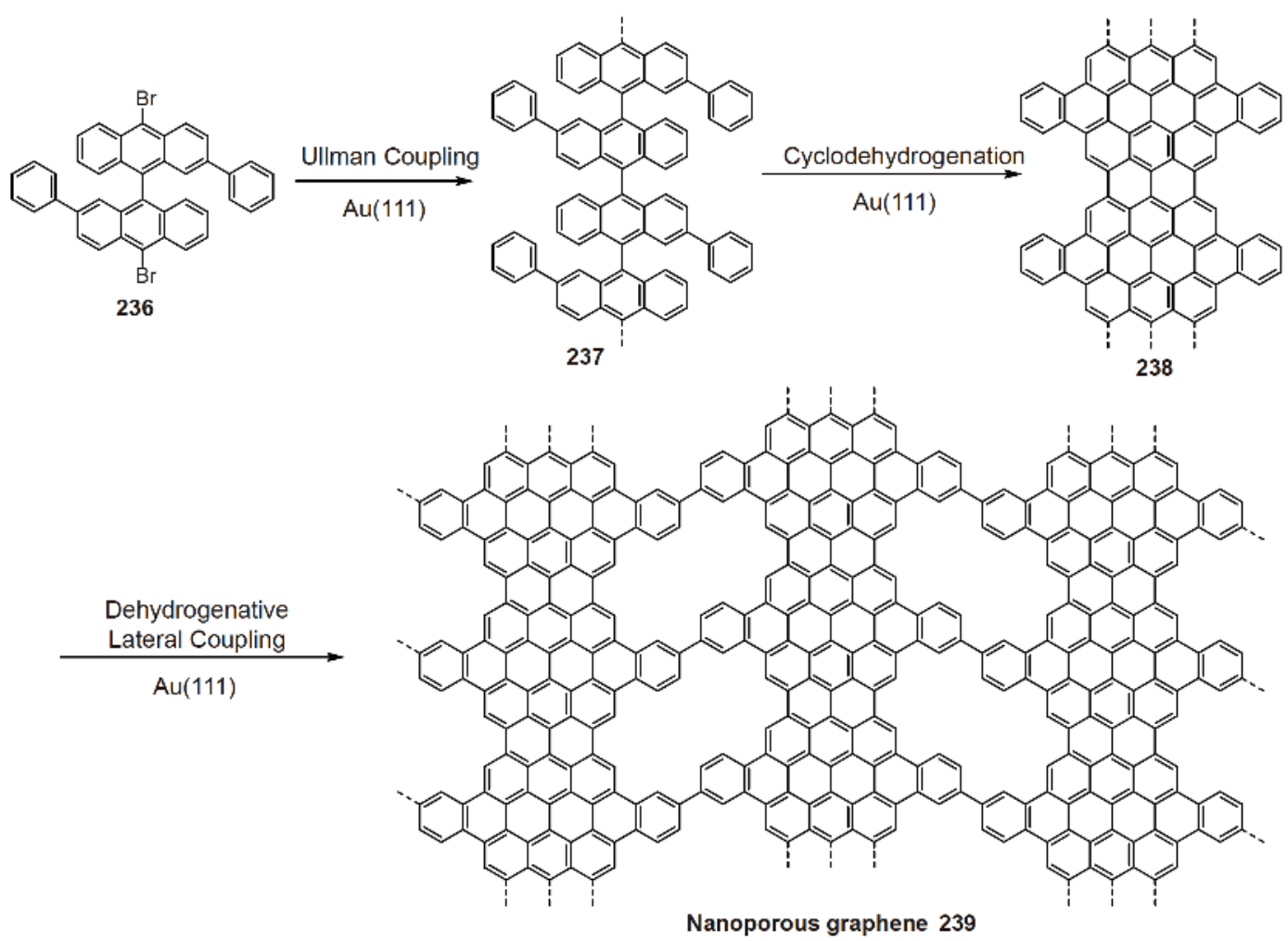

Figure 51 On-surface synthesis of nanoporous graphene 239 via dehydrogenative lateral couplings of obtained GNRs.

electronic, magnetic, and catalytic properties [298]. Precise control of the doping concentration and position is vital to tune the material properties and reveal the structure-property relationships. Unfortunately, ensuring a precise structure of heteroatom-doped graphene at the atomic level is impossible; however, such structural control can be achieved in heteroatom-doped nanographene molecules and GNRs, providing reliable structure-property correlations.

$\mathrm{N}$-doped graphene has attracted enormous attention due to its applications in FET devices, supercapacitors, Li-ion batteries, and fuel cells [299,300]. Besides the issues of doping concentration and position, the type of $\mathrm{N}$-incorporation is another factor due to the existence of pyridinic, pyrrolic, or graphitic nitrogens, which generate significantly different electronic effects. As illustrated in Figure 52, "graphitic" nitrogen refers to $\mathrm{N}$ atoms that replace $\mathrm{C}$ atoms in the graphitic hexagonal lattice; a "pyridinic" nitrogen bonds with two $\mathrm{C}$ atoms either at the edge of graphene or at the edge of internal defects; and a "pyrrolic" nitrogen relates to $\mathrm{N}$ atoms that provide two $p_{\mathrm{z}}$ electrons to the pyrrole-like ring [299,300]. The difficulty to precisely control the type of $\mathrm{N}$ atoms in graphene has prohibited the rational design of materials. For example, N-doped graphene has been a promising metal-free electrocatalyst for the ORR in fuel cells, and has even achieved higher ORR activity compared with the commercial $\mathrm{Pt} / \mathrm{C}$ catalyst in alkaline solutions [301]. However, the local structure of the active sites

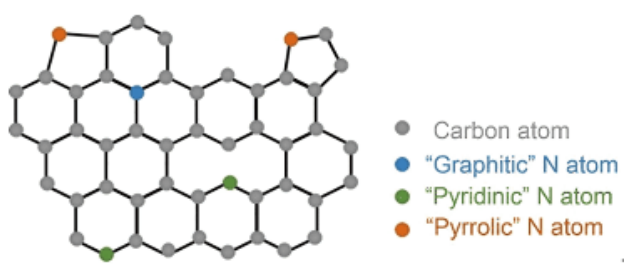

Figure 52 Schematic illustration of various types of nitrogen in N-doped graphene (color online).

remains unclear and the type of nitrogen that is most efficient for ORR is still under debate [302-304]. Therefore, it would be important to synthesize structurally defined nanographene molecules to rationalize not only the catalytic but also the electronic and magnetic properties.

As the first example of pyridinic-N-doped nanographene molecules, $\mathrm{N}$-doped $p$-HBC $\mathbf{2 4 0}$ was synthesized by Draper et al. in 2002 [305] through an oxidative cyclodehydrogenation of the corresponding pyrimidine-substituted hexaarylbenzene precursor (Figure 53). The pyridinic nitrogens rendered PAH $\mathbf{2 4 0}$ relatively electron-deficient and made it a suitable ligand for complexing with metals (e.g., $\mathrm{Pd}^{\mathrm{II}}$ and $\mathrm{Ru}^{\mathrm{II}}$ ) [306]. Draper et al. [307] further installed electron-donating methoxy groups in the precursors to promote the cyclodehydrogenation and synthesized a series of methoxy-functionalized N-doped $p$-HBCs (241-243). Methoxy substituents not only affected the electronic prop- 
erties but also exhibited a supramolecular effect by arranging the molecules in a head-to-tail manner in the crystals.

The studies of these $\mathrm{N}$-doped $p$-HBCs paved the way to GNRs employing pyridinic-N-centers. For example, in 2013, Bronner, Hecht and Tegeder et al. [308] reported the Ndoped chevron-type GNR 245 using 4,4'-(6,11-dibromo-1,4diphenyltriphenylene-2,3-diyl)dipyridine (244) as the monomer on $\mathrm{Au}(111)$ surface (Figure 54(a)). Du and Feng et al. [309] in 2014 revealed the precise structure of N-doped GNR 245 by high-resolution STM. Interestingly, different from the pristine chevron-type GNRs [144], a side-by-side alignment was observed for the N-doped GNRs due to the attractive $\mathrm{N} \cdot \cdots \mathrm{H}$ interactions. Furthermore, different $\mathrm{N}$-doping levels could be precisely controlled in such chevron-type GNRs by employing suitable tetraphenyl triphenylene-based monomers containing one, two, or four nitrogen atoms. The $\mathrm{N}$-doping simultaneously lowered the energy levels of the valence and conduction bands without significant changes in the band gap [305]. Remarkably, in 2014, Müllen and Fasel et al. [310] reported the on-surface synthesis of a GNR 248 composed of alternating $\mathrm{N}$-doped and pristine ribbon segments (Figure 54(b)). The GNR heterojunction occurring in 248 represents the first example of a molecular p-n junction in a single-GNR, exhibiting a band offset of approximately $0.5 \mathrm{eV}$.

Apart from the electronic aspect, the pyridinic nitrogen is also relevant in the context of electrocatalysis, e.g., in the ORR. The aforementioned debate on which type of nitrogen is more efficient for the ORR is mainly based on pyridinic versus graphitic nitrogens, and the mechanism is still unclear [302-304]. In this regard, using structurally well-defined nanographene molecules as models for mechanistic studies of the ORR is important. In 2012, Li et al. [311] synthesized three nanographene molecules featuring a common phena-

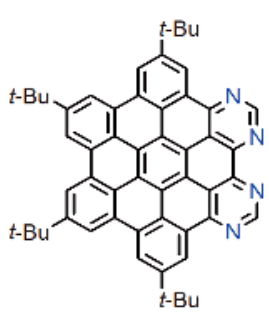

240

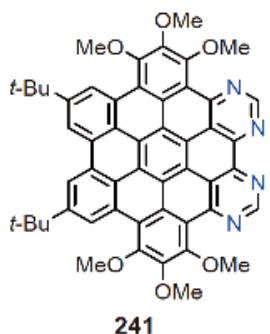

241

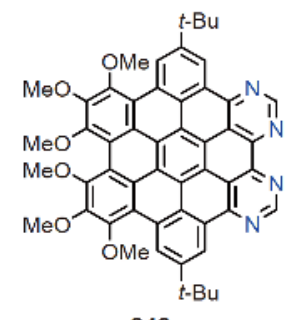

242

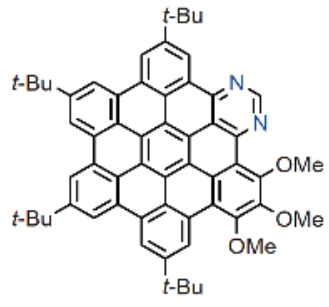

243

Figure 53 Representative examples of pyridinic-N-doped nanograhene molecules ( $p$-HBC derivatives) (color online).

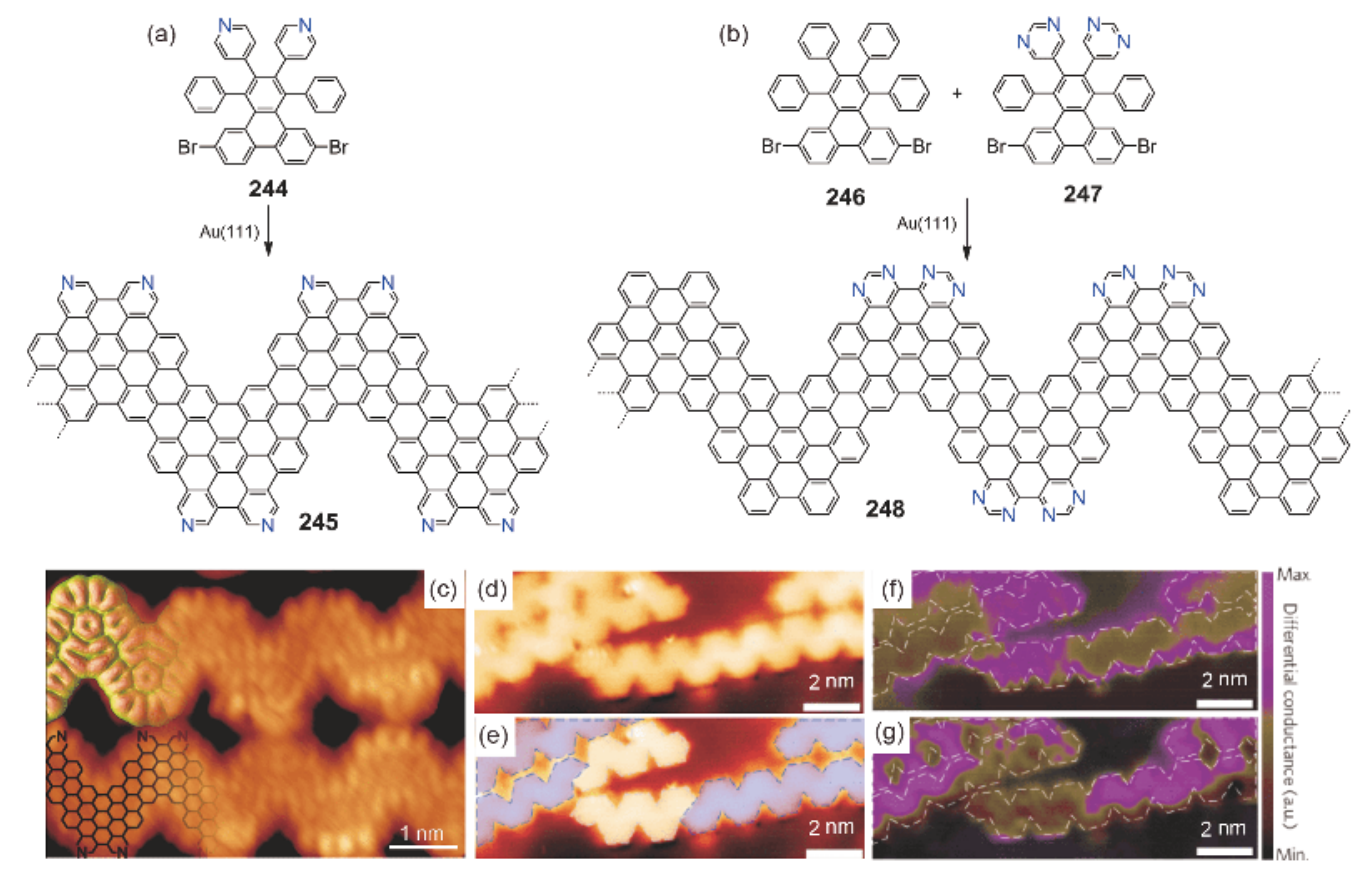

Figure 54 Surface-assisted synthesis of (a) N-doped chevron-type GNR 245 and (b) GNR heterojunction 248. (c) High-resolution STM image of N-doped chevron-type GNR 245, showing an antiparallel alignment to maximize the attractive $\mathrm{N} \cdots \mathrm{H}$ interactions. Reprinted with permission from Ref. [309], copyright (2014) AIP Publishing LLC. (d, e) STM images of N-doped chevron-type GNR 248. The N-doped and non-doped GNR segments are highlighted in blue and light gray dash lines, respectively, in panel (e). (f, g) Differential-conductance (d $I / \mathrm{d} V$ ) maps observed at bias voltages of (f) $-0.35 \mathrm{~V}$ and $(\mathrm{g})-1.65 \mathrm{~V}$. Reprinted with permission from Ref. [310], copyright (2014) Macmillan Publishers Ltd. (color online). 


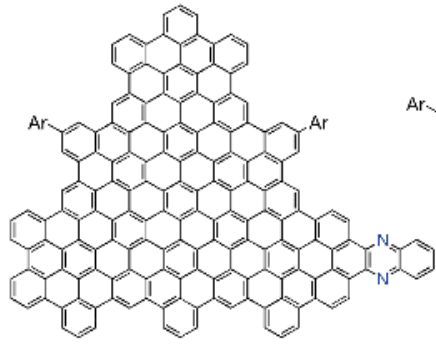

249

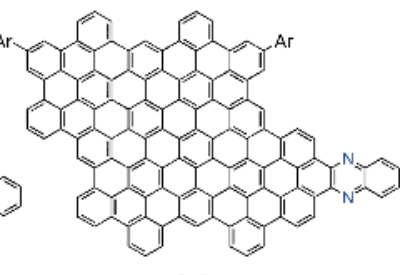

250

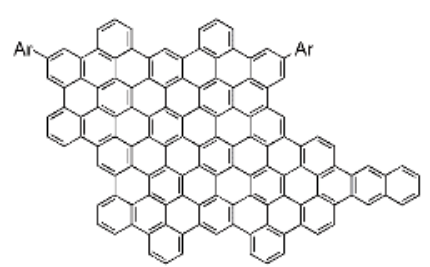

251

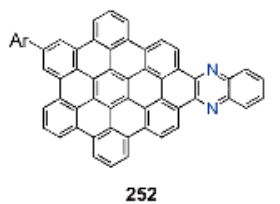

252

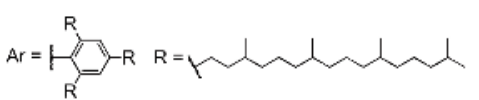

Figure 55 Structures of N-doped and non-doped nanographene molecules (color online).

zine moiety but having different sizes for the ORR studies (Figure 55). A hydrocarbon-based molecule 251 was also synthesized for comparison. Detailed investigations revealed that all the N-doped nanographenes exhibited higher electrocatalytic activities than the hydrocarbon, indicating the important role of N-doping. Moreover, among the pyridinic-
$\mathrm{N}$-doped nanographenes, the larger ones displayed higher activities in the ORR, probably due to the higher HOMO levels, which facilitated the electron transfer to oxygen. Further theoretical studies on compound $\mathbf{2 5 2}$ suggested that the ORR preferred the four-electron pathway in alkaline electrolytes but the two-electron pathway in acidic conditions, consistent with the experimental $\mathrm{pH}$-dependent selectivity observed in the ORR catalyzed by N-doped graphene [312]. This work confirmed the electroactivity of the pyridinic-N atoms, providing clear insights into the more complex N-doped graphene; thus, synthesizing nanographene molecules with different types of nitrogens would be desirable.

From the chemistry point of view, incorporation of pyridinic nitrogens into nanographene molecules does not change the total number of $\pi$-electrons and thus the aromatic nature, whereas introducing graphitic nitrogens would significantly alter the aromaticity and electronic structures. In 2017, Auwärter, Barth, Palma and Müllen et al. [313] explored the synthesis of pyrazine-embedded $p$-HBC, which possessed two more $\pi$-electrons than the carbon analog due to the employment of two graphitic nitrogens in the molecular center (Figure 56(b)). The synthesis was based on a polycyclic azomethine ylide (PAMY) homocoupling starting from dibenzo-9a-azaphenalene salts $\mathbf{2 5 5}$. Through the solution chemistry, dimerization was first performed, followed by an oxidative dehydrogenation to obtain N-doped hexabenzoperylene 257a, but further attempts to cyclodehy-

(a)

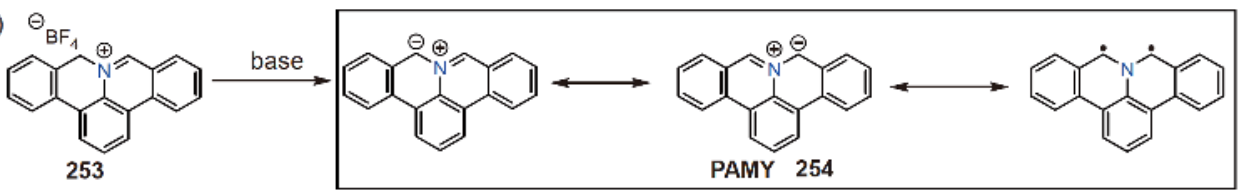

on $\mathrm{Ag}(111)$ surface
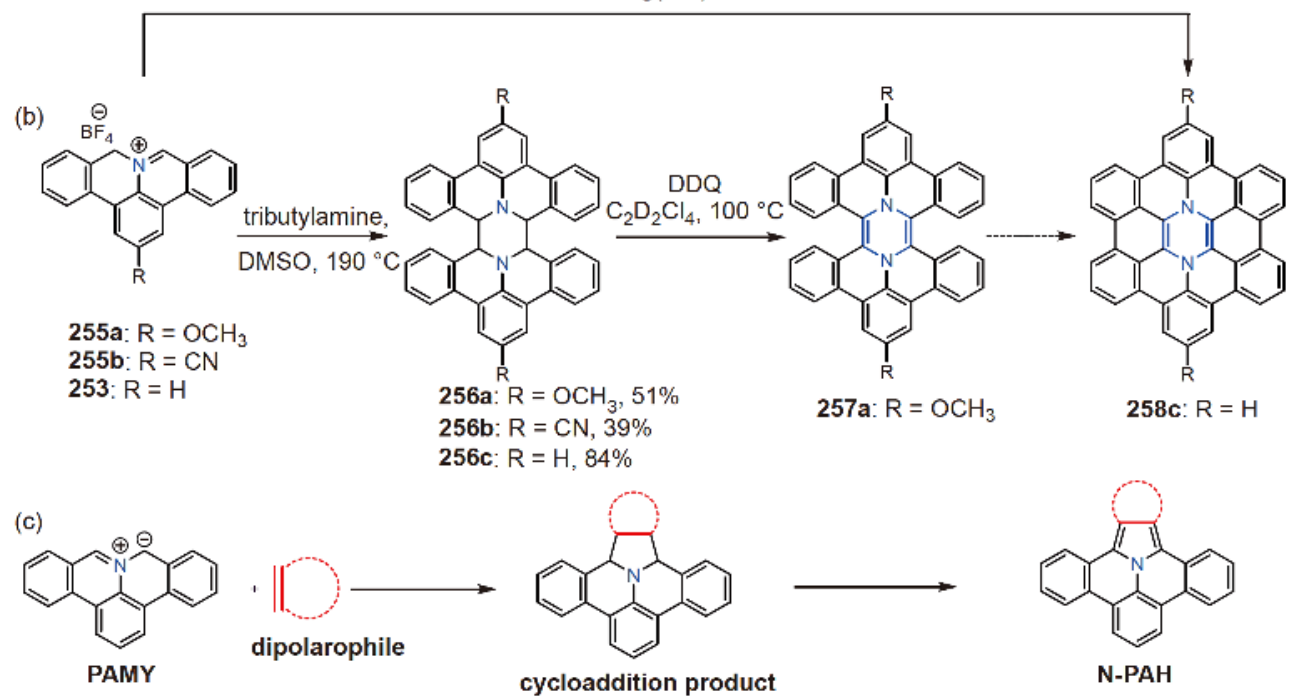

Figure 56 (a) Generation of polycyclic azomethine ylide (PAMY) and its resonance structures; (b) synthesis of pyrazine-embedded partially fused PAH 257a and fully fused $p$-HBC 258c via PAMY homocoupling; (c) PAMY for 1,3-dipolar cycloaddition reactions with dipolarophiles. DMSO: dimethyl sulfoxide; DDQ: 2,3-dichloro-5,6-dicyano-1,4-benzoquinone (color online). 
drogenate 257a were unsuccessful. Therefore, on-surface synthesis under UHV was performed, leading to N-doped $p$ HBC 258c directly from the dibenzo- $9 a$-azaphenalene salt 253 without observing the intermediates.

The PAMY is not only reactive toward dimerization but also a good candidate for 1,3-dipolar cycloaddition reactions with different dipolarophiles, offering a unique route to Ndoped PAHs after oxidation of the cycloaddition product (Figure 56(c)) [314,315].As depicted in Figure 56(a), several resonance structures of the PAMY exist: the ionic structures indicate that the negative charge is equally distributed on the two carbon atoms adjacent to the central nitrogen but the diradical structure also contributes significantly to the ground state. Regarding electrocatalysis, the PAMY could be a possible model of the active sites in N-doped graphene for the ORR as theoretical calculations have revealed that the high charge or spin density of the carbon atoms adjacent to nitrogens is responsible for the catalytic activity [316]. Furthermore, mechanistic studies of the ORR have been exclusively based on a stepwise reaction process either via the two-electron or the more desirable four-electron mechanism (see Figure 57) [317]. By looking at the reactivity of the PAMY toward 1,3-dipolar cycloaddition reactions, we could consider a concerted reaction mechanism for the ORR. On the other hand, discussions on the type of nitrogen, pyridinic-N, graphitic-N, or pyrrolic-N are most relevant for the catalytic activity, which currently gives contradictory conclusions [302-304,318]. In fact, even for the same type of nitrogen, local structural factors (e.g., zigzag or armchair edge configurations, defects, and substituents) have a significant impact on the catalytic activity [319]. Therefore, the study of structurally defined N-doped PAHs could help to

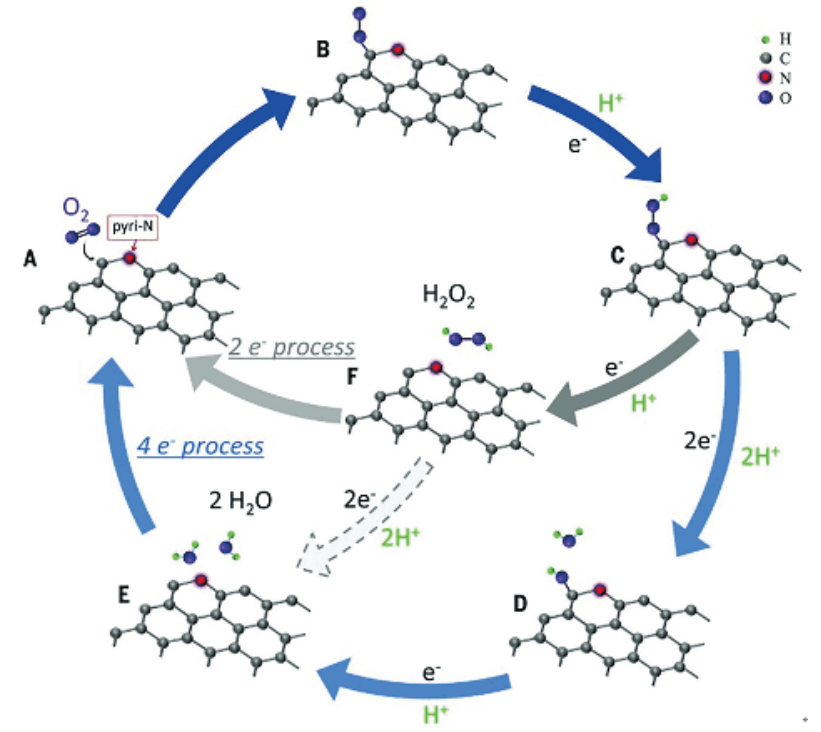

Figure 57 Possible mechanisms of oxygen reduction reaction (ORR) catalyzed by nitrogen-doped carbon materials via two-electron or fourelectron pathway. Reprinted with permission from Ref. [317], Copyright (2016) American Association for the Advancement of Science (color online).

disclose the ORR mechanisms.

Apart from the aforementioned pyridinic and graphitic nitrogens, another common type of $\mathrm{N}$ atoms in graphene structures is pyrrolic nitrogen. In recent years, organic synthesis has promoted the emergence of various pyrrolic Ndoped nanographene molecules (Figure 58). In 2007, Müllen et al. [320] reported the synthesis and properties of hexapyrrolohexaazacoronenes $\mathbf{2 5 9}$, which are isoelectronic analogs of $p$-HBC. The synthesis was also based on oxidative cyclodehydrogenation of hexapyrrolylbenzene pre-

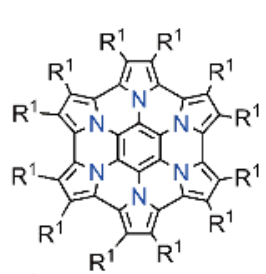

$\mathrm{R}^{1}=\mathrm{H}, \mathrm{Br}, n$-octyl or 4-trifluoromethylphenyl 259

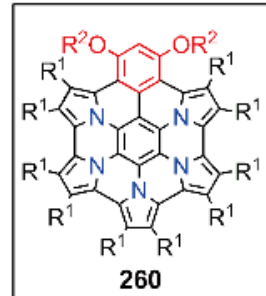

$\mathrm{R}^{1}=4$-trifluoromethylphenyl or $\mathrm{H}$ $\mathrm{R}^{2}=n-\mathrm{C}_{12} \mathrm{H}_{25}, n-\mathrm{C}_{4} \mathrm{H}_{9}$ or $\mathrm{CH}_{3}$
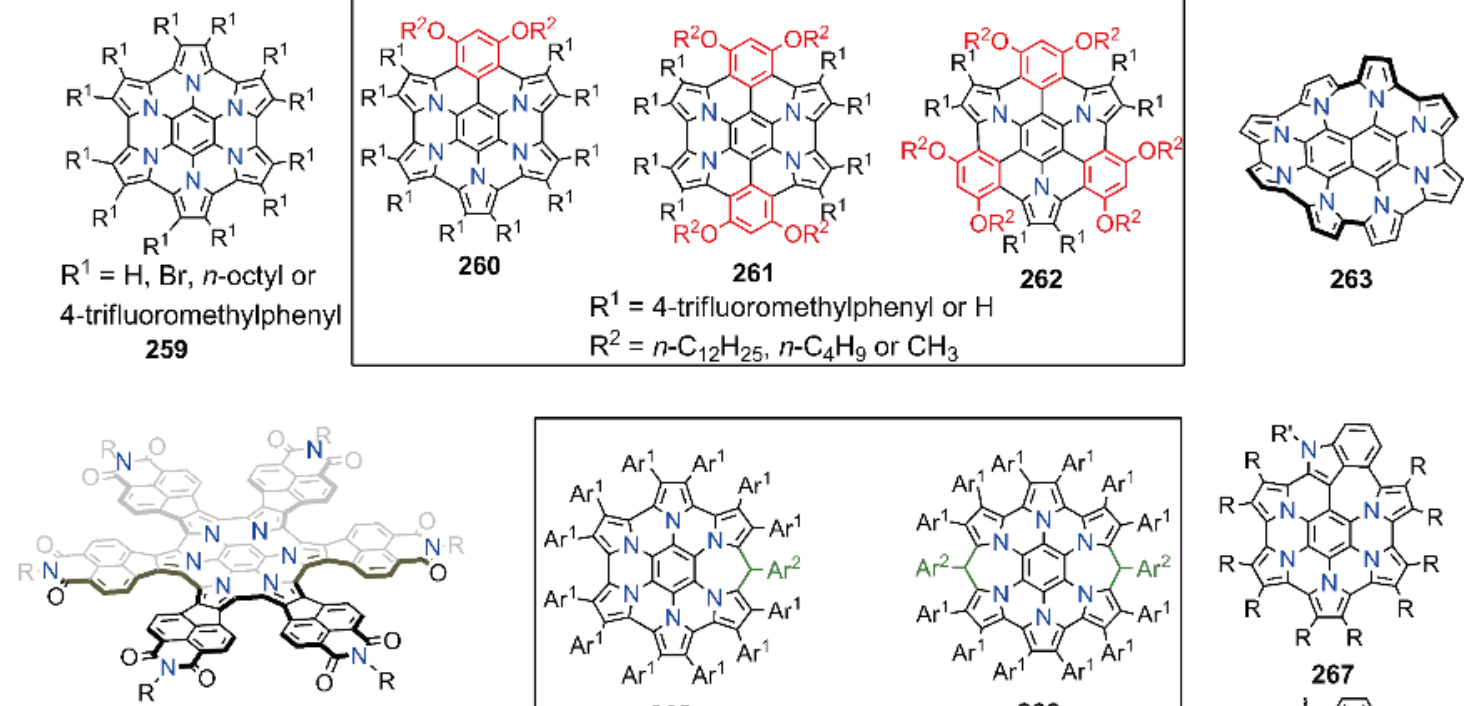

264a: $R=\operatorname{dipp}$ 264b: $R=H$; DFT only

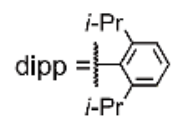

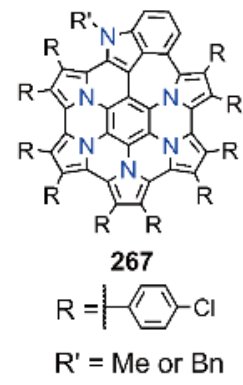

Figure 58 Representative examples of pyrrolic N-doped nanographene molecules (color online). 
cursors, which was previously analyzed by matrix-assisted laser desorption/ionization time-of-flight (MALDI-TOF) mass spectrometry [321]. These electron-rich PAHs exhibited up to four reversible electrochemical oxidations, and their radical cations and dications could be easily generated with chemical oxidants such as $\mathrm{SbCl}_{5}$. In 2013, Takase, Nishinaga and Müllen et al. [322] extended the pyrrole-fused azacoronene family by partly replacing the pyrrole rings with dialkoxybenzenes. These new derivatives 260-262 displayed altered optical, electronic, and redox properties. In particular, an interesting difference appeared in the dicationic state: $\mathbf{2 5 9}^{\mathbf{2 +}}$ and $\mathbf{2 6 0}^{\mathbf{2 +}}$ exhibited a closed-shell character, whereas theoretical studies indicate open-shell structures for $\mathbf{2 6 1} \mathbf{2}^{\mathbf{2 +}}$ and $\mathbf{2 6 2}^{\mathbf{2}}$. The reason is that the spin delocalization was prohibited by the dialkoxybenzenes, thereby causing "weakly interacting" spins.

Efforts have also been made to extend or modify this unique molecular system. In 2018, Takase and Uno et al. [323] synthesized a core-expanded analog by replacing the central benzene with naphthalene, resulting in a curved structure $\mathbf{2 6 3}$ with two N-doped heptagons. In 2016, Stępień et al. [324] described a radially $\pi$-extended hexapyrrolohexaazacoronene 264 by fusing six naphthalene monoimide units on it. This large disc-shaped molecule combined the electron-deficient nature of naphthalene monoimides and the electronrich character of the hexapyrrolohexaazacoronene core, exhibiting a multi-redox behavior with at least 13 oxidation levels. To introduce seven-membered rings, in 2014, Stępień et al. [325] installed saturated methylene bridges in hexapyrrolohexaazacoronenes 265 and 266 to interrupt the peripheral conjugation. Consequently, higher oxidation levels could be easily accessed and a tetracationic species $\mathbf{2 6 6}^{4+}$ could be generated by chemical oxidation with $\mathrm{SbCl}_{5}$. In 2016, the same group [326] reported a new derivative $\mathbf{2 6 7}$ containing a combination of 5-, 6-, and 7-membered rings by oxidative cyclodehydrogenation of an indole-containing precursor. The extended molecule displayed a lower HOMOLUMO gap compared with the pristine hexapyrrolohexaazacoronene. These $\pi$-extensions and modifications have largely enriched the azacoronene family and provided opportunities for discovering new properties.

To access a variety of pyrrolic N-doped nanographene molecules, fusing the peripheral pyrrole derivatives by oxidative cyclodehydrogenation is a straightforward process. Another design concept is to place pyrrole in the center of the molecule to construct $\pi$-extended skeletons (Figure 59). A typical example is azapentabenzocorannulene, which was synthesized independently by two different groups in 2015 . Hiroto and Shinokubo et al. [327] started the synthesis with the oxidation of 9-aminophenanthrene $\mathbf{2 6 8}$, providing tetrabenzocarbazole 269, which was further converted to the Ndoped PAH 272 through stepwise palladium-catalyzed intramolecular cyclizations (Figure 59(a)). Nozaki et al. [328] adopted another synthetic strategy, which was based on 1,3-
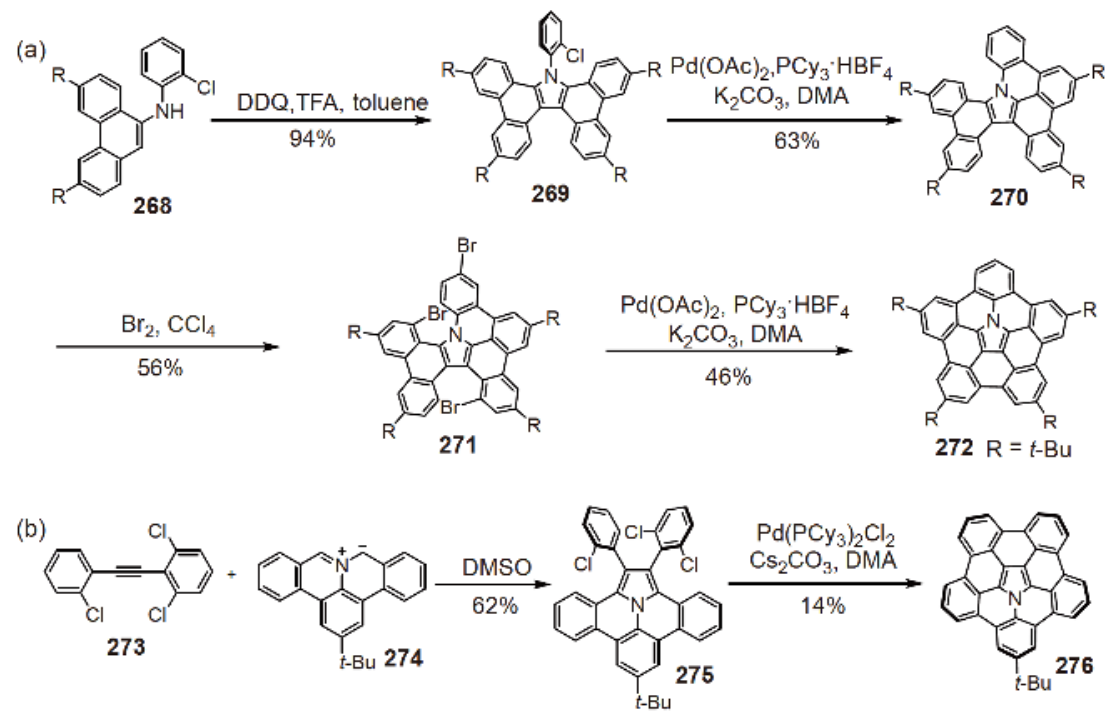

(c)
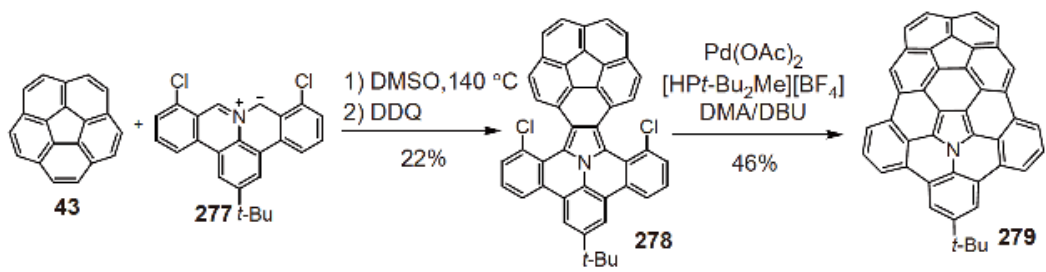

Figure 59 (a) Synthesis of N-doped PAH 272 through stepwise palladium-catalyzed intramolecular cyclizations; (b) synthesis of N-doped PAH 276 via 1,3dipolar cycloaddition of PAMY with diarylacetylene; (c) synthesis of N-doped PAH 279 through 1,3-dipolar cycloaddition of PAMY with corannulene. 
dipolar cycloaddition of PAMY 274 with a diarylethyne 273, followed by palladium-catalyzed intramolecular Heck couplings (Figure 59(b)). Recently in 2018, the same group described the synthesis of an $\pi$-extended azacoranulene $\mathbf{2 7 9}$, which can be considered as a corannulene-azacorannulene hybrid, through 1,3-dipolar cycloaddition of PAMY 277 bearing chloro substituents and subsequent palladium-catalyzed intramolecular cyclizations (Figure 59(c)) [329]. These azacorannulene derivatives exhibited remarkable properties, such as easily accessible radical cationic species due to the embedded pyrrolic nitrogen and tight association with C60 fullerene by virtue of the bowl-shaped $\pi$-surface.

In 2018, Fasel and Gryko et al. [330] reported an N-doped nanographene $\mathbf{2 8 8}$ with the inverse Stone-Wales topology. The target compound was obtained through successive ring closures of the tetraarylpyrrolo[3,2- $b$ ]pyrrole $\mathbf{2 8 0}$ and $\mathbf{2 8 3}$ precursors via a combined in-solution and on-surface synthetic strategy (Figure 60). The precursor for the on-surface synthesis turned out to be essential to the successful formation of the fully fused structure. In the case of precursor 282, surface-assisted cyclodehydrogenation only provided the partially fused product $\mathbf{2 8 6}$, forming only one heptagonal ring. This result required a new design of the precursor, i.e., compound 285, with two heptagons already constructed by solution chemistry. With the new precursor, the cyclodehydrogenation, which generated two new hexagons, proceeded smoothly on the surface to provide the $\mathrm{N}$-doped nanographene $\mathbf{2 8 8}$.
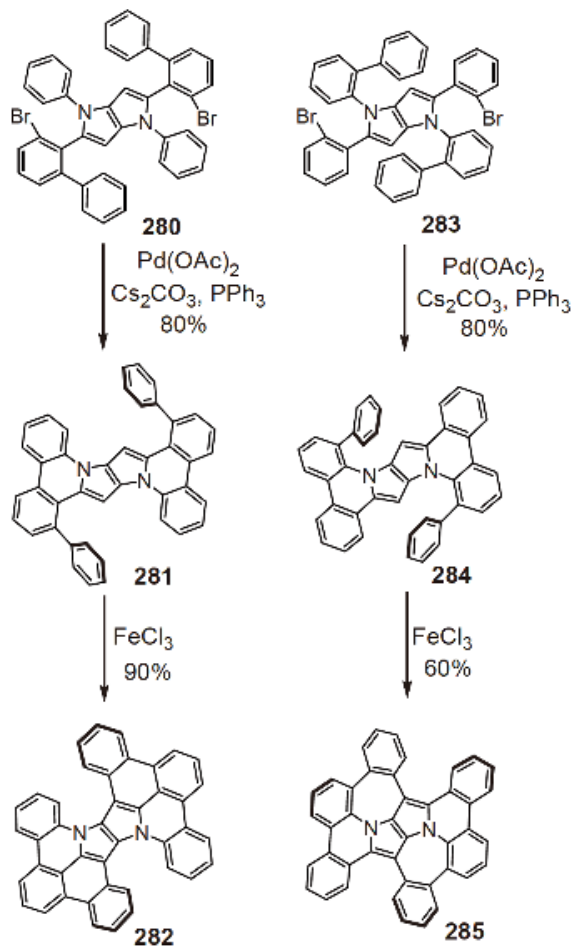

The preceding synthetic achievements have provided a variety of molecular models that can foster the analysis of graphene properties. For instance, doping of graphene with nitrogen atoms has been identified as a promising way to imprint magnetic ordering into graphene; however, the type of $\mathrm{N}$ atoms has exhibited significantly different effects on the magnetic properties of graphene. In 2015, Sachdev and Müllen et al. [331] revealed that introducing pyrrolic nitrogen into the graphene lattice lowered the magnetization compared with that observed for defective graphene (Figure 61(a)). The decrease of the magnetization was attributed to a reduction of the pentagonal carbon radical defects in the presence of the pyrrolic nitrogens. Such a behavior could be explained when looking at the relevant molecular structures, pentabenzocorannulene and its $\mathrm{N}$-doped analogs (272 and 276), which were previously introduced in Figure 59. The hydrocarbon molecule possesses an unpaired electron and is magnetic, whereas the azapentabenzocorannulene has a closed-shell structure and is non-magnetic (Figure 61(b)). In 2017, Otyepka and Zborril et al. [332] demonstrated that graphitic $\mathrm{N}$-doping triggered ferromagnetism in graphene, whereas pyridinic and adsorbed nitrogen (pyrrolic-N not observed in their sample) contributed much less to the ferromagnetic ground state. Theoretical calculations identified a magnetic configuration in which two carbon atoms at para positions of a benzene ring were substituted by two nitrogen atoms (see Figure 61(c)). This structural motif generated significant spin densities and was responsible for the oc-
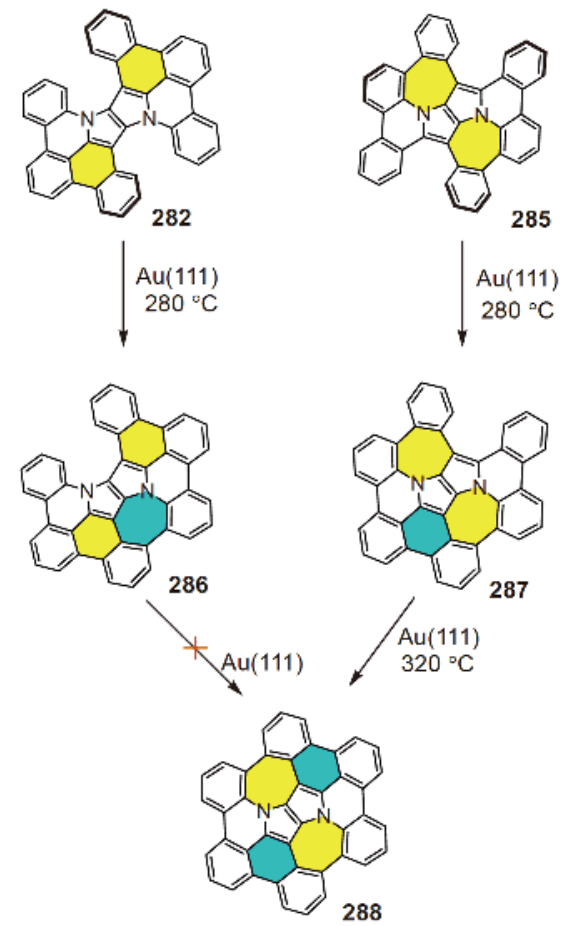

Figure 60 Synthesis of pyrrolic $\mathrm{N}$-doped nanographene $\mathbf{2 8 8}$ by a combination of in-solution and on-surface chemistry. $\mathrm{Pd}(\mathrm{OAc})_{2}$ : palladium(II) acetate (color online). 

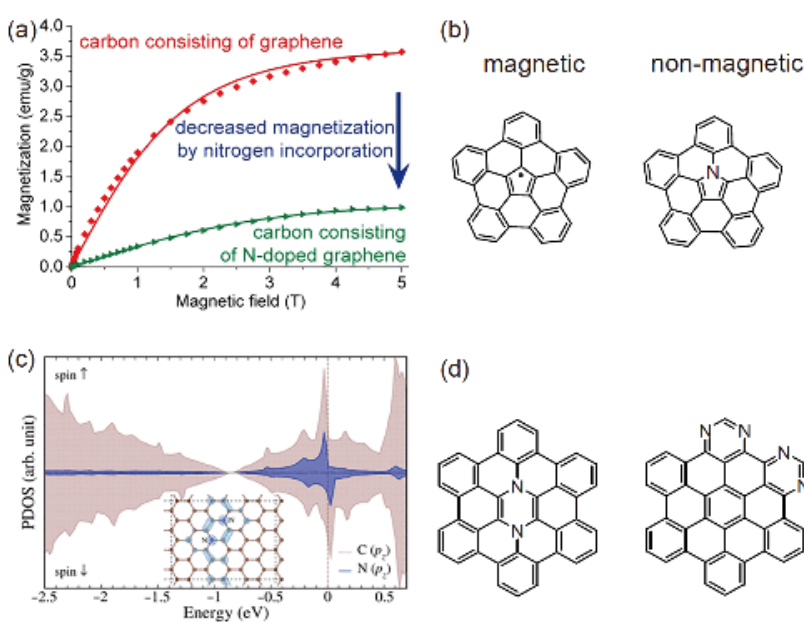

(d)
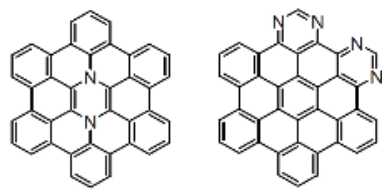

Figure 61 (a) Magnetization curves of graphitic carbon materials with/ without nitrogen. Reprinted with permission from Ref. [331], copyright (2015) American Chemical Society. (b) Origin of observed magnetic properties from a chemical point of view. (c) Partial densities of states calculated for $\mathrm{N}$-doped graphene with graphitic nitrogens embedded in the lattice at para positions. Reprinted with permission from Ref. [332], copyright (2017) American Chemical Society. (d) Relevant graphitic and pyridinic-N-doped $p$-HBCs (color online).

currence of ferromagnetism in graphene. Indeed, a molecular system of such a structure, namely, pyrazine-embedded $p$ HBC 258c, was achieved in 2017 and introduced previously (Figure 56). Detailed investigations of this newly synthesized graphitic $\mathrm{N}$-doped $p$-HBC, as well as the pyridinic and pyrrolic N-doped analogs (240-243 and 259-267, respectively), would offer insights into the role of different $\mathrm{N}$ atoms in modulating the magnetic properties of graphene.

In view of the electronic effect, boron doping represents the other side of the coin with regard to nitrogen doping, as boron has an empty $p_{z}$ orbital compared with carbon and thereby induces p-type doping in graphene [333]. Although B-doped graphene has not been widely studied as the $\mathrm{N}$ doped counterpart, recent advances have demonstrated its promising applications in FET devices [334], Li-ion batteries [335], and supercapacitors [336]. In particular, the Lewis acidity of boron has also facilitated gas adsorption on Bdoped graphene, promoting applications such as toxic gas detection and electrocatalytic oxygen and nitrogen reduction reaction (NRR) [337-339]. However, the lack of structural precision has somewhat obscured the effect of boron doping at the molecular level. Therefore, the synthesis of structurally well-defined boron-doped nanographene molecules is indispensable.

Compared with $\mathrm{N}$-doping, incorporating boron atoms into nanographenes is a more challenging task due to the high intrinsic instability of tri-coordinated organoboranes toward moisture. In 2012, Saito and Yamaguchi et al. [340] disclosed the synthesis of B-doped nanographene molecule 292a with two $B$ atoms incorporated into the carbon framework (Figure 62). The key step in the synthesis was the

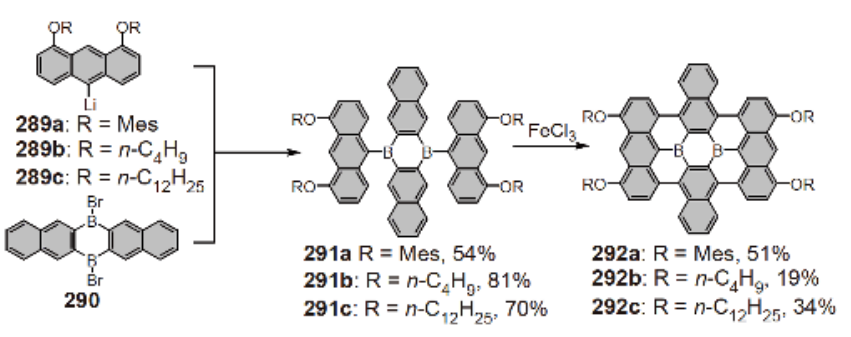

Figure 62 Synthesis of B-doped nanographenes 292.

oxidative cyclodehydrogenation of an anthryl-substituted diborapentacene precursor 291. The planarized product turned out to be stable under ambient conditions and could be handled without any special precautions. A further detailed study on various derivatives 292b and 292c revealed the significant effect of B-doping on the physicochemical properties [341]. The B centers could form coordination complexes with Lewis bases, which changed the absorption and fluorescence properties and molecular conformation. Two-electron reduction could be readily achieved due to the presence of the two B dopants, providing a dianionic species with a triplet ground state. Such unique redox properties also endowed the B-doped nanographenes with good performance as a Li-ion battery electrode material.

Through a similar synthetic strategy, a 9,10-dibora-9,10dihydroanthracene-based monomer 293 was synthesized and employed in the surface-assisted synthesis of B-doped 7AGNRs 294, as independently reported by Kawai et al. [297] as well as Crommie and Fischer et al. [342] in 2015 (Figure 63(a)). Kawai et al. [297] discovered the lateral fusion of these B-doped 7-AGNRs, forming wider 14- and 21-AGNRs (Figure 63(b-d)). The researchers also characterized the precise GNR structures by nc-AFM and found that B atoms displayed a defect-like feature with a darker contrast (more negative frequency shift) due to closer distance of B atoms to the $\mathrm{Au}$ surface. Further STM experiments in conjunction with theoretical calculations revealed the adsorption of nitric oxide (NO) at the boron sites with $\mathrm{N}$ pointing to boron. This finding unambiguously demonstrated the binding behavior of boron due to its Lewis acid character, providing structural proof of the mechanistic analysis of the gas sensing, ORR, and NRR processes.

$\mathrm{B} / \mathrm{N}$ codoping has been an efficient strategy to enhance the electrocatalytic performance of graphene arising from synergistic effects [298,303,343]. Again, molecular nanographenes with $\mathrm{B} / \mathrm{N}$ incorporation could serve as models to unravel the underlying mechanisms. Moreover, BN doping also generates appealing electronic consequences, giving rise to an intermediate between the gapless graphene and insulating hexagonal boron nitride (h-BN). In 2010, Ajayan et al. [344] reported a type of 2D hybrid structure consisting of h-BN and graphene domains by CVD synthesis (Figure 64 (a)). The electrical conductivity can be easily controlled from 

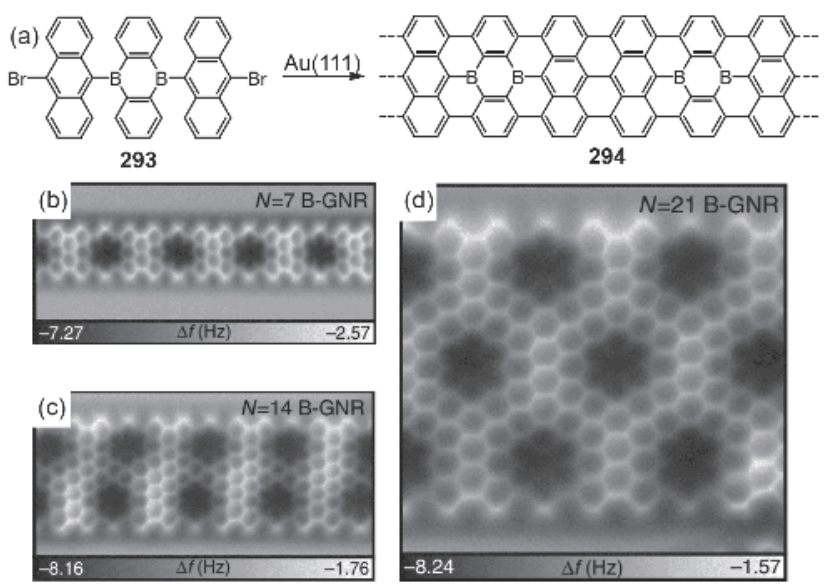

Figure 63 (a) Surface-assisted synthesis of B-doped 7-AGNRs. (b) STM image of B-doped 7-AGNRs. (c, d) Laterally fused 14- and 21-AGNRs, respectively. Reprinted with permission from Ref. [297], copyright (2015) Macmillan Publishers Ltd.
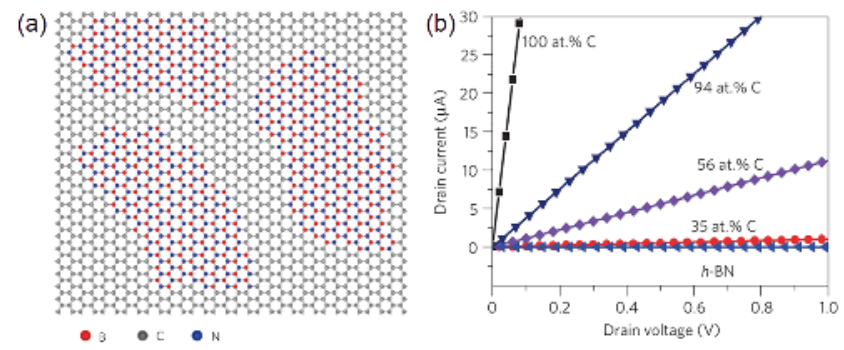

Figure 64 (a) Schematic illustration of hybrid structure consisting of h$\mathrm{BN}$ and graphene domains. (b) Current-voltage $(I-V)$ characteristics of hybrid materials with different carbon contents. Reprinted with permission from Ref. [344], copyright (2010) Macmillan Publishers Ltd. (color online).

insulator (h-BN) to highly conducting (graphene, 100\% C) by tuning the carbon concentration. The carrier mobility of the hybrid structures is in the range of $5-20 \mathrm{~cm}^{2} \mathrm{~V}^{-1} \mathrm{~s}^{-1}$, probably due to the electron scattering at the boundaries between the graphene and h-BN domains (Figure 64(b)). As shown in Figure 64(a), although the ratio between $\mathrm{BN}$ and carbon can be controlled, the phase separation between the graphene and h-BN domains cannot be avoided. Therefore, site-specific $\mathrm{BN}$ doping is desirable to prevent the electron scattering at the boundaries and improve control of the electronic properties.

BN-doped PAHs have been valuable in modeling the sitespecific BN doping of graphene [345]. Furthermore, through appropriate molecular design, intermolecular dehydrogenative fusion could lead to the BN-doped graphene without phase separation of graphene and h-BN domains. In 2014, to establish the synthesis of large BN-doped PAHs, Cao, Wang and Pei et al. [346] reported the first 2D $\pi$-extended BN-doped nanographene molecules 297 by employing thiophene-substituted diazapentacenes $\mathbf{2 9 6}$ as the precursors (Figure 65). Through an electrophilic borylation,

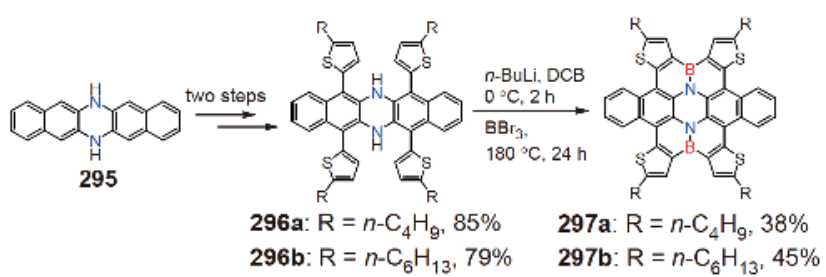

Figure 65 Synthesis of 2D $\pi$-extended BN-doped nanographenes 297 (color online).

two B-N bonds and four B-C bonds were efficiently built up in one step. The molecules displayed two different curved conformations in single crystals and packed into a columnar structure. The formed 1D ribbons of $\mathbf{2 9 7} \mathbf{b}$ were integrated into FET devices, showing a hole mobility of up to $0.23 \mathrm{~cm}^{2} \mathrm{~V}^{-1} \mathrm{~s}^{-1}$ and an on-off ratio of $>10^{4}$. The application of the BN-doped nanographene 297b in organic photovoltaic devices was also investigated [347]. Owing to the supramolecular interaction between the curved $\pi$-surface and commonly used fullerene acceptor $\left(\mathrm{PC}_{71} \mathrm{BM}\right)$, a power conversion efficiency (PCE) of up to $3.12 \%$ was achieved in a binary device despite the large band gap of $297 \mathbf{b}$. In a ternary device, the curved nanographene was found to significantly increase the PCE and enhance the device stability.

In 2018, Hatakeyama et al. [348] reported a one-shot multiple borylation of $N^{1}, N^{1}, N^{3}, N^{3}, N^{5}, N^{5}$-hexakis(4-methylphenyl)-1,3,5-benzenetriamine 298 to achieve BN-doped nanographene molecules. Depending on the borylation conditions, a variety of BN-doped nanographenes 299-301 were synthesized, as detailed in Figure 66. Among them, compound 299 was employed in OLEDs, exhibiting deep blue emission at $460 \mathrm{~nm}$ with an external quantum efficiency of $18.3 \%$ at $1 \mathrm{~cd} \mathrm{~m}^{-2}$. The power of electrophilic borylation was also exploited by Hatakeyama et al. [349] in the synthesis of BN-doped diboratetrabenzocorannulene 304, demonstrating its efficacy even in constructing strained structures (Figure 67).

To achieve site-specific BN-doped GNRs, in 2018, Kawai, Hatakeyama and Foster et al. [350] synthesized a dibenzo[b, e][1,4]azaborinine-based precursor $\mathbf{3 0 5}$ similar to the monomer design for the B-doped GNRs 294, and then subjected it to on-surface polymerization and cyclodehydrogenation (Figure 68). Owing to the asymmetric structure of the precursor arising from the $\mathrm{B} / \mathrm{N}$ positions and terminal iodine/chlorine substituents, two different reaction paths occurred and led to two different segments of GNRs with regard to the BN direction. As the lateral fusion of GNRs has been observed in several studies [164,170,295-297], fusing the current BN-doped GNRs into site-specific BN-doped graphene is possible in the future.

In addition to the aforementioned cases, the recently synthesized borazine-embedded $p$-HBC derivatives $\mathbf{3 1 5}$ and $\mathbf{3 1 9}$ represent a good model for the $2 \mathrm{D}$ heterostructure of h-BN 

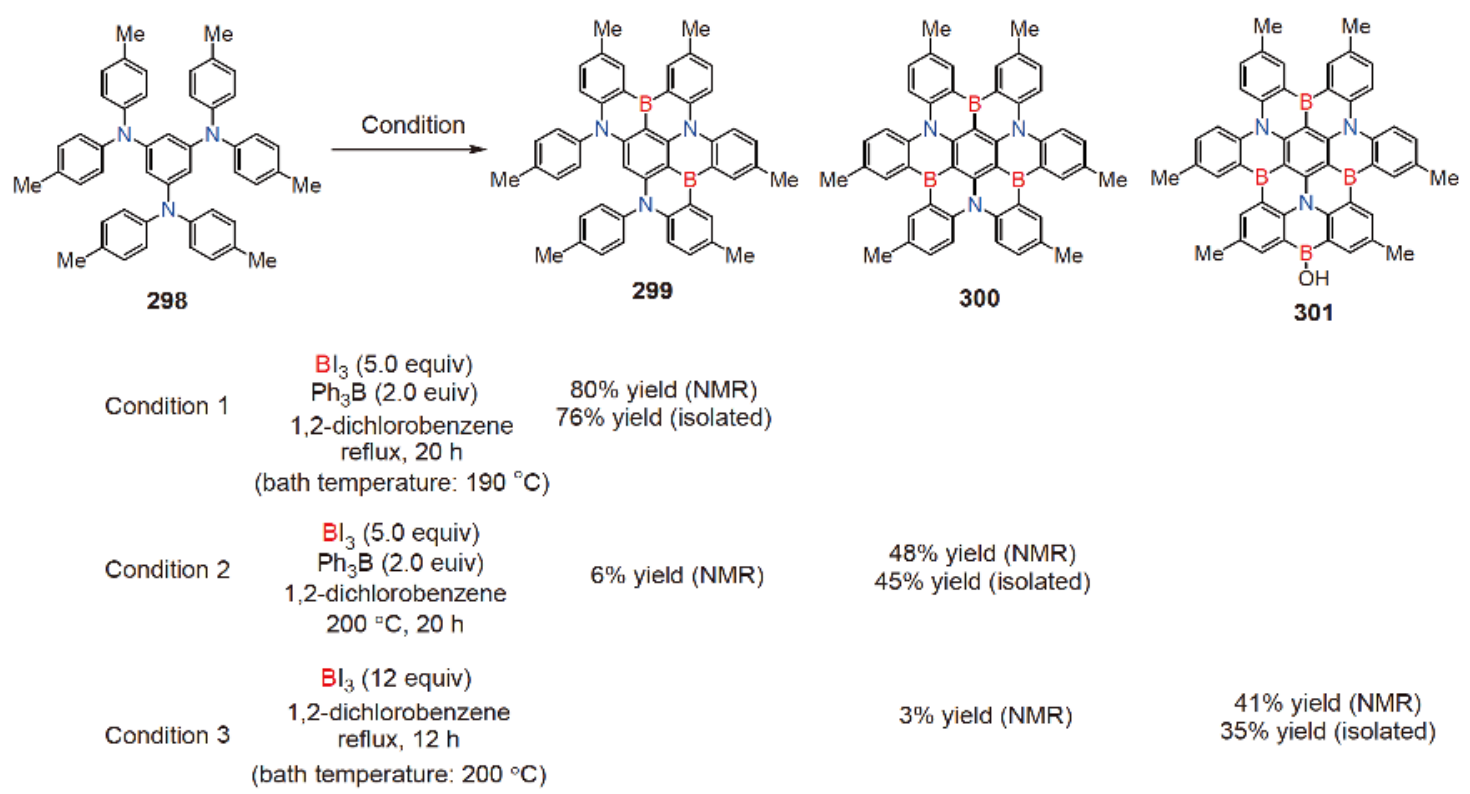

Figure 66 Synthesis of BN-doped nanographenes 299, 300, and 301 by one-shot borylation under different conditions (color online).

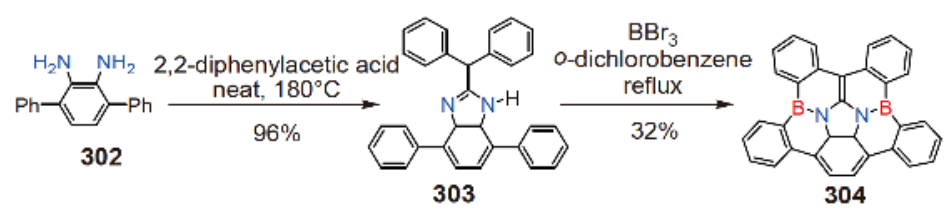

Figure 67 Synthesis of bowl-shaped BN-doped diboratetrabenzocorannulene 304 (color online).
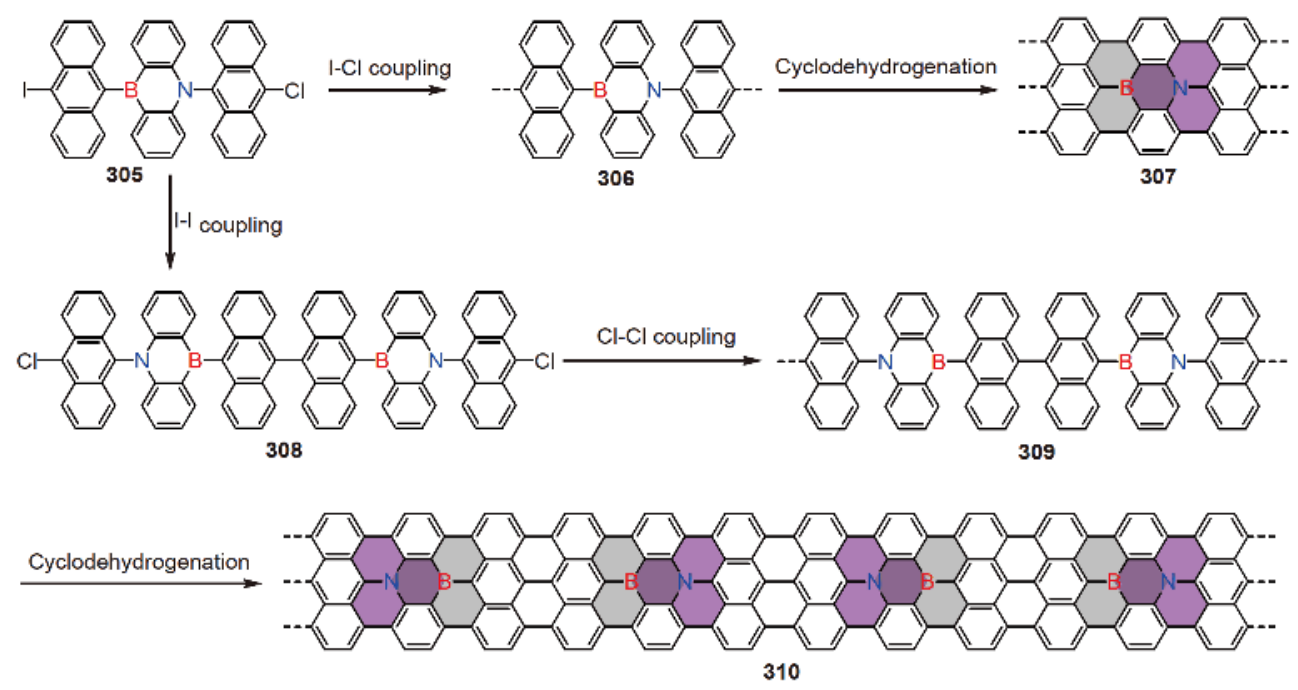

Figure 68 Surface-assisted synthesis of BN-doped GNRs comprising two different segments with regard to BN direction (color online).

and graphene (Figure 69). Bettinger et al. [351] have tried various precursors and methods to access such a skeleton. In 2014 , they subjected the $\mathrm{B}_{3} \mathrm{~N}_{3}$-hexabenzotriphenylene precursor 312 to ring-closure reactions, but the Scholl reactions were unsuccessful and the photocyclization could only close one $\mathrm{C}-\mathrm{C}$ bond to yield partially fused product 313 [351]. In
2015 , they successfully proved the synthesis of $\mathrm{B}_{3} \mathrm{~N}_{3}$-doped $p$-HBC 315 through thermolysis of tris(2-biphenylyl)borazine 314 at $550{ }^{\circ} \mathrm{C}$, although the major product was tetraazatetraborocine derivative 316 [352]. The $\mathrm{B}_{3} \mathrm{~N}_{3}$-doped $p$ HBC 315 was isolated and characterized by mass spectrometry, infrared spectroscopy, solid-state NMR and STM, but 


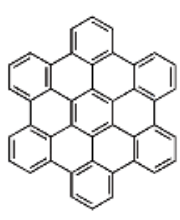

p-HBC 6
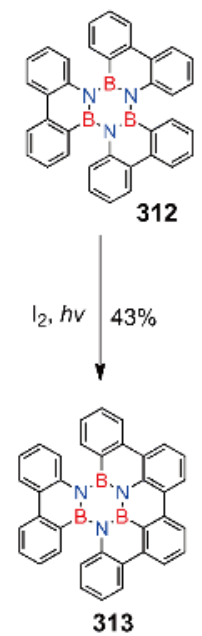

313

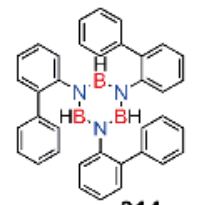

314

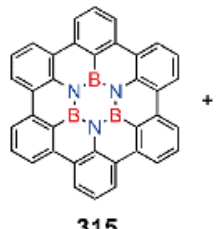

315

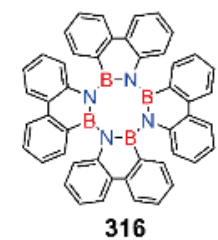

316
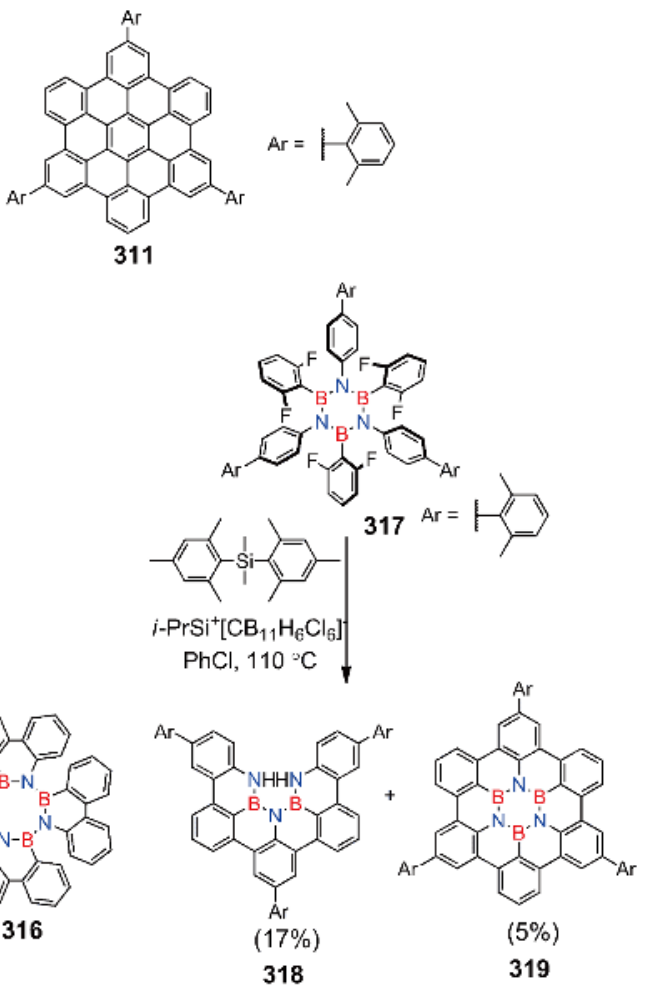

Figure 69 Synthetic efforts toward borazine-embedded $p$-HBC derivatives (315 and 319) (color online).

the poor solubility prohibited in-depth studies on its structure and properties. In 2017, Bonifazi et al. [353] achieved the solution synthesis of a soluble $\mathrm{B}_{3} \mathrm{~N}_{3}$-doped $p$-HBC 319 . Hexaphenylborazine $\mathbf{3 1 7}$ was selected as the precursor but functionalized with six $\mathrm{F}$ atoms to promote the ring closures by Friedel-Crafts-type substitution reaction. The $\mathrm{B}_{3} \mathrm{~N}_{3}$-doped $p$-HBC 319 was obtained in $5 \%$ yield, with the partially fused molecule 318 as the major isolated product in $17 \%$ yield. The synthesis of the soluble $\mathrm{B}_{3} \mathrm{~N}_{3}$-doped $p$-HBC 319 enabled a direct comparison with its all-carbon congener $p$ HBC 311 having the same peripheral substituents. The results pointed toward a dramatic widening of the band gap upon borazine doping, in agreement with the observations in the lateral heterostructure of h-BN and graphene.

\section{Conclusions and perspective}

To reiterate, this review is not intended to focus on the intrinsic value of PAHs but to uncover the strong interactions between the fields of PAHs and graphenes. PAHs have long been studied in different contexts, such as in organic chemistry, industrial dye-stuff production, optoelectronics, astrophysics, or environmental science. Since graphene became a major topic in materials science, it has provided a new angle to study PAHs, and inversely, PAHs have profoundly contributed to the further development of the graphene field. First, we cannot ignore that large PAHs are indeed structu- rally well-defined GQDs, which provide reliable structureproperty correlations and proper control of the material performance. Second, the chemistry of PAHs has promoted the bottom-up synthesis of atomically precise GNRs and invention of emerging GNR-based materials such as topological insulators. Such a bottom-up synthesis protocol has even led to the production of a semiconducting holey graphene with regular hole defects. Finally, various PAHs can serve as model compounds for GNRs to reveal the lengthdependent properties and for graphene to unveil the origin of high electrocatalytic activities together with the emergence of magnetic properties in defective and heteroatom-doped graphene.

Looking forward, we emphasize that PAHs and graphenes should not develop in isolated ways. Many challenges have to be addressed in both fields, such as the efficient synthesis of large PAHs; the search for high-performance electronic, photonic, and spintronic materials; the development of emergent topological insulators; the synthesis of high-quality graphenes on insulating substrates; the precise functionalization of graphene materials; and the understanding of structure-property relationships. Among various future directions, the following points deserve close attention: (1) New design concepts and efficient syntheses in the PAH field could further promote the structural innovation of GQDs and GNRs, which may lead to the discovery of exciting properties. These developments would require not only the expertise of organic chemists but also close collaborations with 
physicists and material scientists. (2) From the synthetic point of view, new methods with high efficiency are always welcome for the PAH synthesis. They are not only valuable to achieve PAH structures that are otherwise difficult to synthesize but also important for the bottom-up synthesis of GNRs, which currently relies on only a few established protocols. (3) Further development of PAHs could facilitate the identification of active sites in electrocatalysis by offering many new PAH models. The graphene research would require new structural models, which could open up new opportunities for material applications. (4) Pushing the size limit of PAHs toward increasingly larger disc structures would narrow the gap between the conventional small PAHs and 2D infinite graphene. Furthermore, direct fusion of PAHs and GNRs into atomically precise graphene would be highly interesting to incorporate holes or heteroatoms in a controlled manner.

Finally, a helpful approach is for physicists and materials scientists to examine the PAH chemistry and for organic chemists to follow the developments in graphene research. We hope that this review can stimulate further interactions between the researchers from both areas and promote a synergistic development of PAHs and graphenes.

\begin{abstract}
Acknowledgements The authors thank all of their distinguished collaborators and research associates who enabled the achievements partly described in this article. This article is a tribute to scientific interaction and its benefit. This work was supported by the European Union Projects GENIUS (ITN-264694), UPGRADE, MoQuaS, and Graphene Flagship (CNECTICT-604391), European Research Council (ERC)-Adv.-Grant 267160 (NANOGRAPH), the Office of Naval Research Basic Research Challenge (BRC) Program (molecular synthesis and characterization), the Max Planck Society, the German Chemical Industry Association, the Alexander von Humboldt Foundation. BASF SE and Samsung are gratefully acknowledged. X.Y. is thankful for a fellowship from the China Scholarship Council.
\end{abstract}

Funding note Open access funding provided by Max Planck Society.

Conflict of interest The authors declare that they have no conflict of interest.

Open Access This article is distributed under the terms of the Creative Commons Attribution 4.0 International License (http://creativecommons. org/licenses/by/4.0/), which permits unrestricted use, distribution, and reproduction in any medium, provided you give appropriate credit to the original author(s) and the source, provide a link to the Creative Commons license, and indicate if changes were made.

1 Hirsch A. Nat Mater, 2010, 9: 868-871

2 Georgakilas V, Perman JA, Tucek J, Zboril R. Chem Rev, 2015, 115: 4744-4822

3 Geim AK, Novoselov KS. Nat Mater, 2007, 6: 183-191

4 Geim AK. Science, 2009, 324: 1530-1534

5 Novoselov KS, Fal'Ko VI, Colombo L, Gellert PR, Schwab MG, Kim K. Nature, 2012, 490: 192-200

6 Wang XY, Narita A, Müllen K. Nat Rev Chem, 2017, 2: 0100

7 Ferrari AC, Bonaccorso F, Fal'ko V, Novoselov KS, Roche S, Bøggild P, Borini S, Koppens FHL, Palermo V, Pugno N, Garrido JA, Sordan R, Bianco A, Ballerini L, Prato M, Lidorikis E, Kivioja J, Marinelli C, Ryhänen T, Morpurgo A, Coleman JN, Nicolosi V,
Colombo L, Fert A, Garcia-Hernandez M, Bachtold A, Schneider GF, Guinea F, Dekker C, Barbone M, Sun Z, Galiotis C, Grigorenko AN, Konstantatos G, Kis A, Katsnelson M, Vandersypen L, Loiseau A, Morandi V, Neumaier D, Treossi E, Pellegrini V, Polini M, Tredicucci A, Williams GM, Hee Hong B, Ahn JH, Min Kim J, Zirath H, van Wees BJ, van der Zant H, Occhipinti L, Di Matteo A, Kinloch IA, Seyller T, Quesnel E, Feng X, Teo K, Rupesinghe N, Hakonen P, Neil SRT, Tannock Q, Löfwander T, Kinaret J. Nanoscale, 2015, 7: 4598-4810

8 Avouris P, Dimitrakopoulos C. Mater Today, 2012, 15: 86-97

9 Allen MJ, Tung VC, Kaner RB. Chem Rev, 2010, 110: 132-145

10 Chen L, Hernandez Y, Feng X, Müllen K. Angew Chem Int Ed, 2012, 51: 7640-7654

11 Peierls RE. Ann Inst Henri Poincare, 1935, 5: 177-222

12 Landau LD. Phys Z Sowjetunion, 1937, 11: 26-35

13 Mermin ND. Phys Rev, 1968, 176: 250-254

14 Novoselov KS, Geim AK, Morozov SV, Jiang D, Zhang Y, Dubonos SV, Grigorieva IV, Firsov AA. Science, 2004, 306: 666-669

15 Schwierz F. Nat Nanotech, 2010, 5: 487-496

16 Narita A, Chen Z, Chen Q, Müllen K. Chem Sci, 2019, 10: 964-975

17 Li X, Wang X, Zhang L, Lee S, Dai H. Science, 2008, 319: 12291232

18 Wang X, Ouyang Y, Li X, Wang H, Guo J, Dai H. Phys Rev Lett, 2008, 100: 206803

19 Jiao L, Wang X, Diankov G, Wang H, Dai H. Nat Nanotech, 2010, 5: 321-325

20 Tour JM. Chem Mater, 2014, 26: 163-171

21 Han MY, Ozyilmaz B, Zhang Y, Kim P. Phys Rev Lett, 2007, 98: 206805

22 Son YW, Cohen ML, Louie SG. Phys Rev Lett, 2006, 97: 216803

23 Han W, Kawakami RK, Gmitra M, Fabian J. Nat Nanotech, 2014, 9: 794-807

24 Cao T, Zhao F, Louie SG. Phys Rev Lett, 2017, 119: 076401

25 Shen J, Zhu Y, Yang X, Li C. Chem Commun, 2012, 48: 3686

26 Zhang Z, Zhang J, Chen N, Qu L. Energy Environ Sci, 2012, 5: 8869

27 Yan X, Li B, Li L. Acc Chem Res, 2013, 46: 2254-2262

28 Müllen K, Wegner G. Electronic Materials: the Oligomer Approach. Weinheim: John Wiley \& Sons, 2008

29 Watson MD, Fechtenkötter A, Müllen K. Chem Rev, 2001, 101: $1267-1300$

30 Wang C, Dong H, Hu W, Liu Y, Zhu D. Chem Rev, 2012, 112: 22082267

31 Dou L, Liu Y, Hong Z, Li G, Yang Y. Chem Rev, 2015, 115: 1263312665

32 Murphy AR, Fréchet JMJ. Chem Rev, 2007, 107: 1066-1096

33 Clar E, Schoental R. Polycyclic Hydrocarbons. Vol. 2. Berlin, Heidelberg: Springer, 1964

34 Armit JW, Robinson R. J Chem Soc Trans, 1925, 127: 1604-1618

35 Payamyar P, King BT, Öttinger HC, Schlüter AD. Chem Commun, 2016, 52: 18-34

36 Zdetsis AD. J Phys Chem C, 2018, 122: 17526-17536

37 Gutzler R, Perepichka DF. J Am Chem Soc, 2013, 135: 16585-16594

38 Li X, Cai W, An J, Kim S, Nah J, Yang D, Piner R, Velamakanni A, Jung I, Tutuc E, Banerjee SK, Colombo L, Ruoff RS. Science, 2009, 324: $1312-1314$

39 Bae S, Kim H, Lee Y, Xu X, Park JS, Zheng Y, Balakrishnan J, Lei T, Ri Kim H, Song YI, Kim YJ, Kim KS, Özyilmaz B, Ahn JH, Hong BH, Iijima S. Nat Nanotech, 2010, 5: 574-578

40 Lin YM, Dimitrakopoulos C, Jenkins KA, Farmer DB, Chiu HY, Grill A, Avouris P. Science, 2010, 327: 662

41 Tzalenchuk A, Lara-Avila S, Kalaboukhov A, Paolillo S, Syväjärvi M, Yakimova R, Kazakova O, Janssen TJBM, Fal'ko V, Kubatkin S. Nat Nanotech, 2010, 5: 186-189

42 Wan X, Chen K, Liu D, Chen J, Miao Q, Xu J. Chem Mater, 2012, 24: 3906-3915

43 Tan YZ, Yang B, Parvez K, Narita A, Osella S, Beljonne D, Feng X, Müllen K. Nat Commun, 2013, 4: 2646 
44 Stein SE, Brown RL. J Am Chem Soc, 1987, 109: 3721-3729

45 Salem L. J Am Chem Soc, 1968, 90: 543-552

46 Dewar MJS. The Molecular Orbital Theory of Organic Chemistry. New York: McGraw-Hill, 1969

47 Würthner F, Saha-Möller CR, Fimmel B, Ogi S, Leowanawat P, Schmidt D. Chem Rev, 2016, 116: 962-1052

48 Chen L, Li C, Müllen K. J Mater Chem C, 2014, 2: 1938-1956

49 Fabian J, Nakazumi H, Matsuoka M. Chem Rev, 1992, 92: 11971226

50 Allamandola LJ, Tielens AGGM, Barker JR. Astrophys J Suppl Ser, 1989, 71: 733-775

51 Tielens AGGM. Annu Rev Astron Astrophys, 2008, 46: 289-337

52 Anthony JE. Chem Rev, 2006, 106: 5028-5048

53 Abdel-Shafy HI, Mansour MSM. Egyptian J Pet, 2016, 25: 107-123

54 Gingras M. Chem Soc Rev, 2013, 42: 968-1006

55 Gingras M, Félix G, Peresutti R. Chem Soc Rev, 2013, 42: 10071050

56 Gingras M. Chem Soc Rev, 2013, 42: 1051-1095

57 Shen Y, Chen CF. Chem Rev, 2012, 112: 1463-1535

58 Song H, Reed MA, Lee T. Adv Mater, 2011, 23: 1583-1608

59 Xiang D, Wang X, Jia C, Lee T, Guo X. Chem Rev, 2016, 116: 43184440

60 Stabel A, Herwig P, Müllen K, Rabe JP. Angew Chem Int Ed, 1995, 34: $1609-1611$

61 Dong L, Wang S, Wang W, Chen C, Lin T, Adisoejoso J, Lin N. Transition metals trigger on-surface ullmann coupling reaction: intermediate, catalyst and template. In: Gourdon A, Ed. On-Surface Synthesis. Advances in Atom and Single Molecule Machines. Cham: Springer International Publishing, 2016. 23-42

62 Sun Q, Zhang R, Qiu J, Liu R, Xu W. Adv Mater, 2018, 30: 1705630

63 Talirz L, Ruffieux P, Fasel R. Adv Mater, 2016, 28: 6222-6231

64 Figueira-Duarte TM, Müllen K. Chem Rev, 2011, 111: 7260-7314

65 Sun M, Müllen K, Yin M. Chem Soc Rev, 2016, 45: 1513-1528

66 Segawa Y, Ito H, Itami K. Nat Rev Mater, 2016, 1: 15002

67 Narita A, Wang XY, Feng X, Müllen K. Chem Soc Rev, 2015, 44: 6616-6643

68 Majewski MA, Stępień M. Angew Chem Int Ed, 2019, 58: 86-116

69 Ito H, Ozaki K, Itami K. Angew Chem Int Ed, 2017, 56: 11144-11164

70 Ito H, Segawa Y, Murakami K, Itami K. J Am Chem Soc, 2019, 141: $3-10$

71 Stępień M, Gońka E, Żyła M, Sprutta N. Chem Rev, 2017, 117: 3479-3716

72 Mas-Torrent M, Rovira C. Chem Rev, 2011, 111: 4833-4856

73 Mei J, Diao Y, Appleton AL, Fang L, Bao Z. J Am Chem Soc, 2013, 135: 6724-6746

74 Wu J, Pisula W, Müllen K. Chem Rev, 2007, 107: 718-747

75 Lei T, Wang JY, Pei J. Chem Mater, 2014, 26: 594-603

76 Sergeyev S, Pisula W, Geerts YH. Chem Soc Rev, 2007, 36: 1902

77 Günes S, Neugebauer H, Sariciftci NS. Chem Rev, 2007, 107: 1324 1338

78 Roy-Mayhew JD, Aksay IA. Chem Rev, 2014, 114: 6323-6348

79 Feng X, Pisula W, Müllen K. Pure Appl Chem, 2009, 81: 2203-2224

80 Rieger R, Müllen K. J Phys Org Chem, 2010, 23: 315-325

81 Sun Z, Ye Q, Chi C, Wu J. Chem Soc Rev, 2012, 41: 7857

82 Grzybowski M, Skonieczny K, Butenschön H, Gryko DT. Angew Chem Int Ed, 2013, 52: 9900-9930

83 Simpson CD, Brand JD, Berresheim AJ, Przybilla L, Räder HJ, Müllen K. Chem Eur J, 2002, 8: 1424-1429

84 Kawasumi K, Zhang Q, Segawa Y, Scott LT, Itami K. Nat Chem, 2013, 5: 739-744

85 Ormsby JL, Black TD, Hilton CL, Bharat CL, King BT. Tetrahedron, 2008, 64: 11370-11378

86 Pradhan A, Dechambenoit P, Bock H, Durola F. J Org Chem, 2013, 78: 2266-2274

87 Dou X, Yang X, Bodwell GJ, Wagner M, Enkelmann V, Müllen K. Org Lett, 2007, 9: 2485-2488

88 Wentrup C. Angew Chem Int Ed, 2017, 56: 14808-14835
89 Tsefrikas VM, Scott LT. Chem Rev, 2006, 106: 4868-4884

90 Scott LT, Boorum MM, McMahon BJ, Hagen S, Mack J, Blank J, Wegner H, de Meijere A. Science, 2002, 295: 1500-1503

91 Scott LT, Jackson EA, Zhang Q, Steinberg BD, Bancu M, Li B. J Am Chem Soc, 2012, 134: 107-110

92 Mallory FB, Mallory CW. Org React, 2004, 30: 1

93 Laarhoven WH. Recl Trav Chim Pays-Bas, 1983, 102: 185-204

94 Meier H. Angew Chem Int Ed Engl, 1992, 31: 1399-1420

95 Xiao S, Myers M, Miao Q, Sanaur S, Pang K, Steigerwald ML, Nuckolls C. Angew Chem Int Ed, 2005, 44: 7390-7394

96 Dössel L, Gherghel L, Feng X, Müllen K. Angew Chem Int Ed, 2011, 50: $2540-2543$

97 Daigle M, Picard-Lafond A, Soligo E, Morin JF. Angew Chem Int Ed, 2016, 55: 2042-2047

98 Jackson EA, Steinberg BD, Bancu M, Wakamiya A, Scott LT. $J$ Am Chem Soc, 2007, 129: 484-485

99 Amsharov KY, Kabdulov MA, Jansen M. Angew Chem Int Ed, 2012, 51: 4594-4597

100 Gross L, Mohn F, Moll N, Liljeroth P, Meyer G. Science, 2009, 325: $1110-1114$

101 Treier M, Pignedoli CA, Laino T, Rieger R, Müllen K, Passerone D, Fasel R. Nat Chem, 2010, 3: 61-67

102 Fort EH, Donovan PM, Scott LT. J Am Chem Soc, 2009, 131: 1600616007

103 Clar E, Zander M. J Chem Soc, 1957, 4616

104 Fort EH, Scott LT. Angew Chem Int Ed, 2010, 49: 6626-6628

105 Some S, Dutta B, Ray JK. Tetrahedron Lett, 2006, 47: 1221-1224

106 Iuliano A, Piccioli P, Fabbri D. Org Lett, 2004, 6: 3711-3714

107 Bonifacio MC, Robertson CR, Jung JY, King BT. J Org Chem, 2005, 70: $8522-8526$

108 Xia Y, Liu Z, Xiao Q, Qu P, Ge R, Zhang Y, Wang J. Angew Chem Int $E d, 2012,51: 5714-5717$

109 Senese DA, Chalifoux AW. Molecules, 2018, 24

110 Yao T, Campo MA, Larock RC. Org Lett, 2004, 6: 2677-2680

111 Goldfinger MB, Crawford KB, Swager TM. J Am Chem Soc, 1997, 119: $4578-4593$

112 Jin T, Zhao J, Asao N, Yamamoto Y. Chem Eur J, 2014, 20: 35543576

113 Donovan PM, Scott LT. J Am Chem Soc, 2004, 126: 3108-3112

114 Shen HC, Tang JM, Chang HK, Yang CW, Liu RS. J Org Chem, 2005, 70: 10113-10116

115 Schuler B, Collazos S, Gross L, Meyer G, Pérez D, Guitián E, Peña D. Angew Chem Int Ed, 2014, 53: 9004-9006

116 Rüdiger EC, Porz M, Schaffroth M, Rominger F, Bunz UHF. Chem Eur J, 2014, 20: 12725-12728

117 Nagao I, Shimizu M, Hiyama T. Angew Chem Int Ed, 2009, 48: 7573-7576

118 Yue W, Gao J, Li Y, Jiang W, Di Motta S, Negri F, Wang Z. J Am Chem Soc, 2011, 133: 18054-18057

119 Dang H, Garcia-Garibay MA. J Am Chem Soc, 2001, 123: 355-356

120 Dang H, Levitus M, Garcia-Garibay MA. J Am Chem Soc, 2002, 124: 136-143

121 Lütke Eversloh C, Avlasevich Y, Li C, Müllen K. Chem Eur J, 2011, 17: $12756-12762$

122 Ozaki K, Kawasumi K, Shibata M, Ito H, Itami K. Nat Commun, 2015, 6: 6251

123 Kato K, Segawa Y, Itami K. Can J Chem, 2016, 95: 329-333

124 Narita A, Feng X, Müllen K. Chem Record, 2015, 15: 295-309

125 Hou ICY, Hu Y, Narita A, Müllen K. Polym J, 2018, 50: 3-20

126 Yang X, Dou X, Rouhanipour A, Zhi L, Räder HJ, Müllen K. J Am Chem Soc, 2008, 130: 4216-4217

127 Schwab MG, Narita A, Hernandez Y, Balandina T, Mali KS, De Feyter S, Feng X, Müllen K. J Am Chem Soc, 2012, 134: 1816918172

128 Wu J, Gherghel L, Watson MD, Li J, Wang Z, Simpson CD, Kolb U, Müllen K. Macromolecules, 2003, 36: 7082-7089

129 Shifrina ZB, Averina MS, Rusanov AL, Wagner M, Müllen K. 
Macromolecules, 2000, 33: 3525-3529

130 Narita A, Feng X, Hernandez Y, Jensen SA, Bonn M, Yang H, Verzhbitskiy IA, Casiraghi C, Hansen MR, Koch AHR, Fytas G, Ivasenko O, Li B, Mali KS, Balandina T, Mahesh S, De Feyter S, Müllen K. Nat Chem, 2013, 6: 126-132

131 Konnerth R, Cervetti C, Narita A, Feng X, Müllen K, Hoyer A, Burghard M, Kern K, Dressel M, Bogani L. Nanoscale, 2015, 7: 12807-12811

132 Soavi G, Dal Conte S, Manzoni C, Viola D, Narita A, Hu Y, Feng X, Hohenester U, Molinari E, Prezzi D, Müllen K, Cerullo G. Nat Commun, 2016, 7: 11010

133 Verzhbitskiy IA, Corato MD, Ruini A, Molinari E, Narita A, Hu Y, Schwab MG, Bruna M, Yoon D, Milana S, Feng X, Müllen K, Ferrari AC, Casiraghi C, Prezzi D. Nano Lett, 2016, 16: 3442-3447

134 Zhao S, Rondin L, Delport G, Voisin C, Beser U, Hu Y, Feng X, Müllen K, Narita A, Campidelli S, Lauret JS. Carbon, 2017, 119: 235-240

135 El Gemayel M, Narita A, Dössel LF, Sundaram RS, Kiersnowski A, Pisula W, Hansen MR, Ferrari AC, Orgiu E, Feng X, Müllen K, Samorì P. Nanoscale, 2014, 6: 6301-6314

136 Abbas AN, Liu G, Narita A, Orosco M, Feng X, Müllen K, Zhou C. $J$ Am Chem Soc, 2014, 136: 7555-7558

137 Ivanov I, Hu Y, Osella S, Beser U, Wang HI, Beljonne D, Narita A, Müllen K, Turchinovich D, Bonn M. J Am Chem Soc, 2017, 139: 7982-7988

138 Jensen SA, Ulbricht R, Narita A, Feng X, Müllen K, Hertel T, Turchinovich D, Bonn M. Nano Lett, 2013, 13: 5925-5930

139 Huang Y, Mai Y, Beser U, Teyssandier J, Velpula G, van Gorp H, Straasø LA, Hansen MR, Rizzo D, Casiraghi C, Yang R, Zhang G, Wu D, Zhang F, Yan D, De Feyter S, Müllen K, Feng X. J Am Chem Soc, 2016, 138: 10136-10139

140 Yang W, Lucotti A, Tommasini M, Chalifoux WA. J Am Chem Soc, 2016, 138: 9137-9144

141 Schwab MG, Narita A, Osella S, Hu Y, Maghsoumi A, Mavrinsky A, Pisula W, Castiglioni C, Tommasini M, Beljonne D, Feng X, Müllen K. Chem Asian J, 2015, 10: 2134-2138

142 Li G, Yoon KY, Zhong X, Wang J, Zhang R, Guest JR, Wen J, Zhu XY, Dong G. Nat Commun, 2018, 9: 1687

143 Li G, Yoon KY, Zhong X, Zhu X, Dong G. Chem Eur J, 2016, 22: 9116-9120

144 Cai J, Ruffieux P, Jaafar R, Bieri M, Braun T, Blankenburg S, Muoth M, Seitsonen AP, Saleh M, Feng X, Müllen K, Fasel R. Nature, 2010, 466: 470-473

145 Zhang H, Lin H, Sun K, Chen L, Zagranyarski Y, Aghdassi N, Duhm S, Li Q, Zhong D, Li Y, Müllen K, Fuchs H, Chi L. J Am Chem Soc, 2015, 137: 4022-4025

146 Sun K, Ji P, Zhang H, Niu K, Li L, Chen A, Li Q, Müllen K, Chi L. Faraday Discuss, 2017, 204: 297-305

147 Sun Q, Zhang C, Li Z, Kong H, Tan Q, Hu A, Xu W. J Am Chem Soc, 2013, 135: 8448-8451

148 Chen Z, Zhang W, Palma CA, Lodi Rizzini A, Liu B, Abbas A, Richter N, Martini L, Wang XY, Cavani N, Lu H, Mishra N, Coletti C, Berger R, Klappenberger F, Kläui M, Candini A, Affronte M, Zhou C, de Renzi V, del Pennino U, Barth JV, Räder HJ, Narita A, Feng X, Müllen K. J Am Chem Soc, 2016, 138: 15488-15496

149 Sakaguchi H, Kawagoe Y, Hirano Y, Iruka T, Yano M, Nakae T. Adv Mater, 2014, 26: 4134-4138

150 Yang S, Lohe MR, Müllen K, Feng X. Adv Mater, 2016, 28: 62136221

151 Lavin-Lopez MP, Valverde JL, Sanchez-Silva L, Romero A. Ind Eng Chem Res, 2016, 55: 845-855

152 Gao H, Hu G. RSC Adv, 2016, 6: 10132-10143

153 Yi M, Shen Z. J Mater Chem A, 2015, 3: 11700-11715

154 Yazdi G, Iakimov T, Yakimova R. Crystals, 2016, 6: 53

155 Wang H, Yu G. Adv Mater, 2016, 28: 4956-4975

156 Li X, Colombo L, Ruoff RS. Adv Mater, 2016, 28: 6247-6252

157 Chen X, Wu B, Liu Y. Chem Soc Rev, 2016, 45: 2057-2074
158 Jiang L, Niu T, Lu X, Dong H, Chen W, Liu Y, Hu W, Zhu D. J Am Chem Soc, 2013, 135: 9050-9054

159 Liang T, Kong Y, Chen H, Xu M. Chin J Chem, 2016, 34: 32-40

160 Magda GZ, Jin X, Hagymási I, Vancsó P, Osváth Z, Nemes-Incze P, Hwang C, Biró LP, Tapasztó L. Nature, 2014, 514: 608-611

161 Talirz L, Söde H, Dumslaff T, Wang S, Sanchez-Valencia JR, Liu J, Shinde P, Pignedoli CA, Liang L, Meunier V, Plumb NC, Shi M, Feng X, Narita A, Müllen K, Fasel R, Ruffieux P. ACS Nano, 2017, 11: $1380-1388$

162 Jordan RS, Li YL, Lin CW, McCurdy RD, Lin JB, Brosmer JL, Marsh KL, Khan SI, Houk KN, Kaner RB, Rubin Y. J Am Chem Soc, 2017, 139: 15878-15890

163 Jänsch D, Ivanov I, Zagranyarski Y, Duznovic I, Baumgarten M, Turchinovich D, Li C, Bonn M, Müllen K. Chem Eur J, 2017, 23 : 4870-4875

164 Chen Z, Wang HI, Bilbao N, Teyssandier J, Prechtl T, Cavani N, Tries A, Biagi R, de Renzi V, Feng X, Kläui M, de Feyter S, Bonn M, Narita A, Müllen K. J Am Chem Soc, 2017, 139: 9483-9486

165 Jordan RS, Wang Y, McCurdy RD, Yeung MT, Marsh KL, Khan SI, Kaner RB, Rubin Y. Chem, 2016, 1: 78-90

166 Kimouche A, Ervasti MM, Drost R, Halonen S, Harju A, Joensuu PM, Sainio J, Liljeroth P. Nat Commun, 2015, 6: 10177

167 Basagni A, Sedona F, Pignedoli CA, Cattelan M, Nicolas L, Casarin M, Sambi M. J Am Chem Soc, 2015, 137: 1802-1808

168 Chen YC, de Oteyza DG, Pedramrazi Z, Chen C, Fischer FR, Crommie MF. ACS Nano, 2013, 7: 6123-6128

169 Xu X, Zhang Z, Qiu L, Zhuang J, Zhang L, Wang H, Liao C, Song H, Qiao R, Gao P, Hu Z, Liao L, Liao Z, Yu D, Wang E, Ding F, Peng H, Liu K. Nat Nanotech, 2016, 11: 930-935

170 Huang H, Wei D, Sun J, Wong SL, Feng YP, Neto AHC, Wee ATS. Sci Rep, 2012, 2: 983

171 Yang L, Park CH, Son YW, Cohen ML, Louie SG. Phys Rev Lett, 2007, 99: 186801

172 Son YW, Cohen ML, Louie SG. Nature, 2006, 444: 347-349

173 Ruffieux P, Wang S, Yang B, Sánchez-Sánchez C, Liu J, Dienel T, Talirz L, Shinde P, Pignedoli CA, Passerone D, Dumslaff T, Feng X, Müllen K, Fasel R. Nature, 2016, 531: 489-492

174 Jia X, Campos-Delgado J, Terrones M, Meunier V, Dresselhaus MS. Nanoscale, 2011, 3: 86-95

175 Girit CO, Meyer JC, Erni R, Rossell MD, Kisielowski C, Yang L, Park CH, Crommie MF, Cohen ML, Louie SG, Zettl A. Science, 2009, 323: 1705-1708

176 Dumslaff T, Yang B, Maghsoumi A, Velpula G, Mali KS, Castiglioni C, de Feyter S, Tommasini M, Narita A, Feng X, Müllen K. J Am Chem Soc, 2016, 138: 4726-4729

177 Gröning O, Wang S, Yao X, Pignedoli CA, Borin Barin G, Daniels C, Cupo A, Meunier V, Feng X, Narita A, Müllen K, Ruffieux P, Fasel R. Nature, 2018, 560: 209-213

178 Rizzo DJ, Veber G, Cao T, Bronner C, Chen T, Zhao F, Rodriguez H, Louie SG, Crommie MF, Fischer FR. Nature, 2018, 560: 204-208

179 Anthony JE. Angew Chem Int Ed, 2008, 47: 452-483

180 Anthony JE, Brooks JS, Eaton DL, Parkin SR. J Am Chem Soc, 2001, 123: 9482-9483

181 Dorel R, Echavarren AM. Eur J Org Chem, 2017, 2017(1): 14-24

182 Herwig PT, Müllen K. Adv Mater, 1999, 11: 480-483

183 Mondal R, Shah BK, Neckers DC. J Am Chem Soc, 2006, 128: 9612 9613

184 Mondal R, Tonshoff C, Khon D, Neckers DC, Bettinger HF. J Am Chem Soc, 2009, 131: 14281-14289

185 Tönshoff C, Bettinger HF. Angew Chem Int Ed, 2010, 49: 4125-4128

186 Krüger J, García F, Eisenhut F, Skidin D, Alonso JM, Guitián E, Pérez D, Cuniberti G, Moresco F, Peña D. Angew Chem Int Ed, 2017, 56: 11945-11948

187 Zuzak R, Dorel R, Kolmer M, Szymonski M, Godlewski S, Echavarren AM. Angew Chem Int Ed, 2018, 57: 10500-10505

188 Scholl R, Mansfeld J. Ber Dtsch Chem Ges, 1910, 43: 1734-1746

189 Scholl R, Seer C, Weitzenböck R. Ber Dtsch Chem Ges, 1910, 43: 


\section{2-2209}

190 Clar E. Chem Ber, 1948, 81: 52-63

191 Clar E. Chem Ber, 1949, 82: 46-60

192 Ajayakumar MR, Fu Y, Ma J, Hennersdorf F, Komber H, Weigand JJ, Alfonsov A, Popov AA, Berger R, Liu J, Müllen K, Feng X. J Am Chem Soc, 2018, 140: 6240-6244

193 Ni Y, Gopalakrishna TY, Phan H, Herng TS, Wu S, Han Y, Ding J, Wu J. Angew Chem Int Ed, 2018, 57: 9697-9701

194 Rogers C, Chen C, Pedramrazi Z, Omrani AA, Tsai HZ, Jung HS, Lin S, Crommie MF, Fischer FR. Angew Chem Int Ed, 2015, 54: 15143-15146

195 Zeng W, Qi Q, Wu J. Eur J Org Chem, 2017, 2018: 7

196 Zeng W, Phan H, Herng TS, Gopalakrishna TY, Aratani N, Zeng Z, Yamada H, Ding J, Wu J. Chem, 2017, 2: 81-92

197 Konishi A, Hirao Y, Matsumoto K, Kurata H, Kishi R, Shigeta Y, Nakano M, Tokunaga K, Kamada K, Kubo T. J Am Chem Soc, 2013, 135: $1430-1437$

198 Konishi A, Hirao Y, Nakano M, Shimizu A, Botek E, Champagne B, Shiomi D, Sato K, Takui T, Matsumoto K, Kurata H, Kubo T. J Am Chem Soc, 2010, 132: 11021-11023

199 Wang S, Talirz L, Pignedoli CA, Feng X, Müllen K, Fasel R, Ruffieux P. Nat Commun, 2016, 7: 11507

200 Talirz L, Söde H, Cai J, Ruffieux P, Blankenburg S, Jafaar R, Berger R, Feng X, Müllen K, Passerone D, Fasel R, Pignedoli CA. J Am Chem Soc, 2013, 135: 2060-2063

201 Paternò GM, Chen Q, Wang XY, Liu J, Motti SG, Petrozza A, Feng X, Lanzani G, Müllen K, Narita A, Scotognella F. Angew Chem Int Ed, 2017, 56: 6753-6757

202 Bellunato A, Arjmandi Tash H, Cesa Y, Schneider GF. ChemPhysChem, 2016, 17: 785-801

203 Suenaga K, Koshino M. Nature, 2010, 468: 1088-1090

204 Zhang X, Xin J, Ding F. Nanoscale, 2013, 5: 2556-2569

205 Clar E, Stephen JF. Tetrahedron, 1965, 21: 467-470

206 Ball M, Zhong Y, Wu Y, Schenck C, Ng F, Steigerwald M, Xiao S, Nuckolls C. Acc Chem Res, 2015, 48: 267-276

207 Liu J, Li BW, Tan YZ, Giannakopoulos A, Sanchez-Sanchez C, Beljonne D, Ruffieux P, Fasel R, Feng X, Müllen K. J Am Chem Soc, 2015, 137: 6097-6103

208 Pradhan A, Dechambenoit P, Bock H, Durola F. Angew Chem Int Ed, 2011, 50: 12582-12585

209 Luo J, Xu X, Mao R, Miao Q. J Am Chem Soc, 2012, 134: 1379613803

210 Li C, Yang Y, Miao Q. Chem Asian J, 2018, 13: 884-894

211 Lin WB, Li M, Fang L, Chen CF. Chin Chem Lett, 2018, 29: 40-46

212 Berezhnaia V, Roy M, Vanthuyne N, Villa M, Naubron JV, Rodriguez J, Coquerel Y, Gingras M. J Am Chem Soc, 2017, 139: 18508-18511

213 Hosokawa T, Takahashi Y, Matsushima T, Watanabe S, Kikkawa S, Azumaya I, Tsurusaki A, Kamikawa K. J Am Chem Soc, 2017, 139 : $18512-18521$

214 Fujikawa T, Segawa Y, Itami K. J Am Chem Soc, 2015, 137: 7763 7768

215 Zhu Y, Xia Z, Cai Z, Yuan Z, Jiang N, Li T, Wang Y, Guo X, Li Z, Ma S, Zhong D, Li Y, Wang J. J Am Chem Soc, 2018, 140: 42224226

216 Wang Y, Yin Z, Zhu Y, Gu J, Li Y, Wang J. Angew Chem Int Ed, 2019, 58: 587-591

217 Schuster NJ, Paley DW, Jockusch S, Ng F, Steigerwald ML, Nuckolls C. Angew Chem Int Ed, 2016, 55: 13519-13523

218 Evans PJ, Ouyang J, Favereau L, Crassous J, Fernández I, Perles J, Martín N. Angew Chem Int Ed, 2018, 57: 6774-6779

219 Nakakuki Y, Hirose T, Sotome H, Miyasaka H, Matsuda K. J Am Chem Soc, 2018, 140: 4317-4326

220 Daigle M, Miao D, Lucotti A, Tommasini M, Morin JF. Angew Chem Int $E d, 2017,56:$ 6213-6217

221 Georgakilas V, Otyepka M, Bourlinos AB, Chandra V, Kim N, Kemp KC, Hobza P, Zboril R, Kim KS. Chem Rev, 2012, 112: 6156-
6214

222 Englert JM, Dotzer C, Yang G, Schmid M, Papp C, Gottfried JM, Steinrück HP, Spiecker E, Hauke F, Hirsch A. Nat Chem, 2011, 3: 279-286

223 Chua CK, Pumera M. Chem Soc Rev, 2013, 42: 3222

224 Eigler S, Hirsch A. Angew Chem Int Ed, 2014, 53: 7720-7738

225 Chen D, Feng H, Li J. Chem Rev, 2012, 112: 6027-6053

226 Fogel Y, Kastler M, Wang Z, Andrienko D, Bodwell GJ, Müllen K. J Am Chem Soc, 2007, 129: 11743-11749

227 Zhou C, Chen S, Lou J, Wang J, Yang Q, Liu C, Huang D, Zhu T. Nanoscale Res Lett, 2014, 9: 26

228 Pumera M, Wong CHA. Chem Soc Rev, 2013, 42: 5987

229 Feng W, Long P, Feng Y, Li Y. Adv Sci, 2016, 3: 1500413

230 Karlický F, Kumara Ramanatha Datta K, Otyepka M, Zbořil R. ACS Nano, 2013, 7: 6434-6464

231 Elias DC, Nair RR, Mohiuddin TMG, Morozov SV, Blake P, Halsall MP, Ferrari AC, Boukhvalov DW, Katsnelson MI, Geim AK, Novoselov KS. Science, 2009, 323: 610-613

232 Savchenko A. Science, 2009, 323: 589-590

233 Yang Z, Sun Y, Alemany LB, Narayanan TN, Billups WE. J Am Chem Soc, 2012, 134: 18689-18694

234 Watson MD, Debije MG, Warman JM, Müllen K. J Am Chem Soc, 2004, 126: 766-771

235 Li B, Zhou L, Wu D, Peng H, Yan K, Zhou Y, Liu Z. ACS Nano, 2011, 5: 5957-5961

236 Wu J, Xie L, Li Y, Wang H, Ouyang Y, Guo J, Dai H. J Am Chem Soc, 2011, 133: 19668-19671

237 Liu YM, Hou H, Zhou YZ, Zhao XJ, Tang C, Tan YZ, Müllen K. Nat Commun, 2018, 9: 1901

238 Tan YZ, Osella S, Liu Y, Yang B, Beljonne D, Feng X, Müllen K. Angew Chem Int Ed, 2015, 54: 2927-2931

239 Cao J, Liu YM, Jing X, Yin J, Li J, Xu B, Tan YZ, Zheng N. J Am Chem Soc, 2015, 137: 10914-10917

240 Wu ZS, Tan YZ, Zheng S, Wang S, Parvez K, Qin J, Shi X, Sun C, Bao X, Feng X, Müllen K. J Am Chem Soc, 2017, 139: 4506-4512

241 Dong R, Pfeffermann M, Skidin D, Wang F, Fu Y, Narita A, Tommasini M, Moresco F, Cuniberti G, Berger R, Müllen K, Feng X. $J$ Am Chem Soc, 2017, 139: 2168-2171

242 Keerthi A, Radha B, Rizzo D, Lu H, Diez Cabanes V, Hou ICY, Beljonne D, Cornil J, Casiraghi C, Baumgarten M, Müllen K, Narita A. J Am Chem Soc, 2017, 139: 16454-16457

243 Dössel LF, Kamm V, Howard IA, Laquai F, Pisula W, Feng X, Li C, Takase M, Kudernac T, de Feyter S, Müllen K. J Am Chem Soc, 2012, 134: 5876-5886

244 Slota M, Keerthi A, Myers WK, Tretyakov E, Baumgarten M, Ardavan A, Sadeghi H, Lambert CJ, Narita A, Müllen K, Bogani L. Nature, 2018, 557: 691-695

245 Pesin D, MacDonald AH. Nat Mater, 2012, 11: 409-416

246 Rogers C, Perkins WS, Veber G, Williams TE, Cloke RR, Fischer FR. J Am Chem Soc, 2017, 139: 4052-4061

247 Joshi D, Hauser M, Veber G, Berl A, Xu K, Fischer FR. J Am Chem Soc, 2018, 140: 9574-9580

248 González-Herrero H, Gómez-Rodríguez JM, Mallet P, Moaied M, Palacios JJ, Salgado C, Ugeda MM, Veuillen JY, Yndurain F, Brihuega I. Science, 2016, 352: 437-441

249 Araujo PT, Terrones M, Dresselhaus MS. Mater Today, 2012, 15: 98-109

250 Banhart F, Kotakoski J, Krasheninnikov AV. ACS Nano, 2011, 5: 2641

251 Liu L, Qing M, Wang Y, Chen S. J Mater Sci Tech, 2015, 31: 599606

252 Jing N, Xue Q, Ling C, Shan M, Zhang T, Zhou X, Jiao Z. RSC Adv, 2012, 2: 9124

253 Mortazavi B, Ahzi S. Carbon, 2013, 63: 460-470

254 Hashimoto A, Suenaga K, Gloter A, Urita K, Iijima S. Nature, 2004, 430: $870-873$

255 Stone AJ, Wales DJ. Chem Phys Lett, 1986, 128: 501-503 
256 Thrower PA. Chem Phys Carbon, 1969, 5: 217-319

257 Huang PY, Ruiz-Vargas CS, van der Zande AM, Whitney WS, Levendorf MP, Kevek JW, Garg S, Alden JS, Hustedt CJ, Zhu Y, Park J, McEuen PL, Muller DA. Nature, 2011, 469: 389-392

258 Yazyev OV, Louie SG. Phys Rev B, 2010, 81: 195420

259 Lahiri J, Lin Y, Bozkurt P, Oleynik II, Batzill M. Nat Nanotech, 2010, 5: 326-329

260 Yazyev OV, Louie SG. Nat Mater, 2010, 9: 806-809

261 Singh R, Kroll P. J Phys: Condens Matter, 2009, 21: 196002

262 Ma Y, Lehtinen PO, Foster AS, Nieminen RM. New J Phys, 2004, 6: 68

263 Valencia AM, Caldas MJ. Phys Rev B, 2017, 96: 125431

264 Kotakoski J, Meyer JC, Kurasch S, Santos-Cottin D, Kaiser U, Krasheninnikov AV. Phys Rev B, 2011, 83: 245420

265 Meyer JC, Kisielowski C, Erni R, Rossell MD, Crommie MF, Zettl A. Nano Lett, 2008, 8: 3582-3586

266 Robertson AW, Allen CS, Wu YA, He K, Olivier J, Neethling J, Kirkland AI, Warner JH. Nat Commun, 2012, 3: 1144

267 Tang M, Colombo L, Zhu J, Diaz de La Rubia T. Phys Rev B, 1997, 55: $14279-14289$

268 Lusk MT, Carr LD. Phys Rev Lett, 2008, 100: 175503

269 Lusk MT, Wu DT, Carr LD. Phys Rev B, 2010, 81: 155444

270 Anderson AG, Daugs ED, Kao LG, Wang JF. J Org Chem, 1986, 51: 2961-2965

271 Vogel E, Markowitz G, Schmalstieg L, Itô S, Breuckmann R, Roth WR. Angew Chem Int Ed, 1984, 23: 719-720

272 Becker BC, Huber W, Schnieders C, Müllen K. Chem Ber, 1983, 14: no

273 Nestoros E, Stuparu MC. Chem Commun, 2018, 54: 6503-6519

274 Zhu J, Huang Y, Mei W, Zhao C, Zhang C, Zhang J, Amiinu IS, Mu S. Angew Chem Int Ed, 2019, 58: 3859-3864

275 Liu J, Osella S, Ma J, Berger R, Beljonne D, Schollmeyer D, Feng X, Müllen K. J Am Chem Soc, 2016, 138: 8364-8367

276 Mishra S, Lohr TG, Pignedoli CA, Liu J, Berger R, Urgel JI, Müllen K, Feng X, Ruffieux P, Fasel R. ACS Nano, 2018, 12: 11917-11927

277 Yamamoto K, Harada T, Nakazaki M, Naka T, Kai Y, Harada S, Kasai N. J Am Chem Soc, 1983, 105: 7171-7172

278 Yamamoto K, Saitho Y, Iwaki D, Ooka T. Angew Chem Int Ed Engl, 1991, 30: 1173-1174

279 Cheung KY, Xu X, Miao Q. J Am Chem Soc, 2015, 137: 3910-3914

280 Pun SH, Chan CK, Luo J, Liu Z, Miao Q. Angew Chem Int Ed, 2017, 57: 1581-1586

281 Fernández-García JM, Evans PJ, Medina Rivero S, Fernández I, García-Fresnadillo D, Perles J, Casado J, Martín N. J Am Chem Soc, 2018, 140: 17188-17196

282 Feng C-N, Kuo M-Y, Wu Y-T. Angew Chem Int Ed, 2013, 52: 77917794

283 Sakamoto Y, Suzuki T. J Am Chem Soc, 2013, 135: 14074-14077

284 Miller RW, Duncan AK, Schneebeli ST, Gray DL, Whalley AC. Chem Eur J, 2014, 20: 3705-3711

285 Cheung KY, Chan CK, Liu Z, Miao Q. Angew Chem Int Ed, 2017, 56: 9003-9007

286 Tang C, Wang HF, Chen X, Li BQ, Hou TZ, Zhang B, Zhang Q, Titirici MM, Wei F. Adv Mater, 2016, 28: 6845-6851

287 Yan X, Jia Y, Yao X. Chem Soc Rev, 2018, 47: 7628-7658

288 Liu M, Liu M, She L, Zha Z, Pan J, Li S, Li T, He Y, Cai Z, Wang J, Zheng Y, Qiu X, Zhong D. Nat Commun, 2017, 8: 14924

289 Lin Y, Liao Y, Chen Z, Connell JW. Mater Res Lett, 2017, 5: 209234

290 Yang J, Ma M, Li L, Zhang Y, Huang W, Dong X. Nanoscale, 2014, 6: 13301-13313

291 Bai J, Zhong X, Jiang S, Huang Y, Duan X. Nat Nanotech, 2010, 5: 190-194

292 Liang X, Jung YS, Wu S, Ismach A, Olynick DL, Cabrini S, Bokor J. Nano Lett, 2010, 10: 2454-2460

293 Beser U, Kastler M, Maghsoumi A, Wagner M, Castiglioni C, Tommasini M, Narita A, Feng X, Müllen K. J Am Chem Soc, 2016,
138: 4322-4325

294 Bieri M, Treier M, Cai J, Aït-Mansour K, Ruffieux P, Gröning O, Gröning P, Kastler M, Rieger R, Feng X, Müllen K, Fasel R. Chem Commun, 2009, 58: 6919

295 Moreno C, Vilas-Varela M, Kretz B, Garcia-Lekue A, Costache MV, Paradinas M, Panighel M, Ceballos G, Valenzuela SO, Peña D, Mugarza A. Science, 2018, 360: 199-203

296 Merino-Díez N, Garcia-Lekue A, Carbonell-Sanromà E, Li J, Corso M, Colazzo L, Sedona F, Sánchez-Portal D, Pascual JI, de Oteyza DG. ACS Nano, 2017, 11: 11661-11668

297 Kawai S, Saito S, Osumi S, Yamaguchi S, Foster AS, Spijker P, Meyer E. Nat Commun, 2015, 6: 8098

298 Wang X, Sun G, Routh P, Kim DH, Huang W, Chen P. Chem Soc Rev, 2014, 43: 7067-7098

299 Wang H, Maiyalagan T, Wang X. ACS Catal, 2012, 2: 781-794

300 Xu H, Ma L, Jin Z. J Energy Chem, 2018, 27: 146-160

301 Liang HW, Zhuang X, Brüller S, Feng X, Müllen K. Nat Commun, 2014, 5: 4973

302 Zhang J, Chen G, Müllen K, Feng X. Adv Mater, 2018, 30: 1800528

303 Hu C, Dai L. Adv Mater, 2019, 31: 1804672

304 Yang L, Shui J, Du L, Shao Y, Liu J, Dai L, Hu Z. Adv Mater, 2019, 31: 1804799

305 Draper SM, Gregg DJ, Madathil R. J Am Chem Soc, 2002, 124: 3486-3487

306 Draper SM, Gregg DJ, Schofield ER, Browne WR, Duati M, Vos JG, Passaniti P. J Am Chem Soc, 2004, 126: 8694-8701

307 Wijesinghe LP, Lankage BS, Máille GMÓ, Perera SD, Nolan D, Wang L, Draper SM. Chem Commun, 2014, 50: 10637-10640

308 Bronner C, Stremlau S, Gille M, Brauße F, Haase A, Hecht S, Tegeder P. Angew Chem Int Ed, 2013, 52: 4422-4425

309 Zhang Y, Zhang Y, Li G, Lu J, Lin X, Du S, Berger R, Feng X, Müllen K, Gao HJ. Appl Phys Lett, 2014, 105: 023101

310 Cai J, Pignedoli CA, Talirz L, Ruffieux P, Söde H, Liang L, Meunier V, Berger R, Li R, Feng X, Müllen K, Fasel R. Nat Nanotech, 2014, 9: 896-900

311 Li Q, Zhang S, Dai L, Li L. J Am Chem Soc, 2012, 134: 1893218935

312 Noffke BW, Li Q, Raghavachari K, Li LS. J Am Chem Soc, 2016, 138: 13923-13929

313 Wang XY, Richter M, He Y, Björk J, Riss A, Rajesh R, Garnica M, Hennersdorf F, Weigand JJ, Narita A, Berger R, Feng X, Auwärter W, Barth JV, Palma CA, Müllen K. Nat Commun, 2017, 8: 1948

314 Berger R, Giannakopoulos A, Ravat P, Wagner M, Beljonne D, Feng X, Müllen K. Angew Chem Int Ed, 2014, 53: 10520-10524

315 Berger R, Wagner M, Feng X, Müllen K. Chem Sci, 2015, 6: 436441

316 Zhang L, Xia Z. J Phys Chem C, 2011, 115: 11170-11176

317 Guo D, Shibuya R, Akiba C, Saji S, Kondo T, Nakamura J. Science, 2016, 351: 361-365

318 Singh SK, Takeyasu K, Nakamura J. Adv Mater, 2019, 31: 1804297

319 Wang T, Chen ZX, Chen YG, Yang LJ, Yang XD, Ye JY, Xia HP, Zhou ZY, Sun SG. ACS Energy Lett, 2018, 3: 986-991

320 Takase M, Enkelmann V, Sebastiani D, Baumgarten M, Müllen K. Angew Chem Int Ed, 2007, 46: 5524-5527

321 Lazerges M, Jouini M, Hapiot P, Guiriec P, Lacaze PC. J Phys Chem A, 2003, 107: 5042-5048

322 Takase M, Narita T, Fujita W, Asano MS, Nishinaga T, Benten H, Yoza K, Müllen K. J Am Chem Soc, 2013, 135: 8031-8040

323 Oki K, Takase M, Mori S, Shiotari A, Sugimoto Y, Ohara K, Okujima T, Uno H. J Am Chem Soc, 2018, 140: 10430-10434

324 Żyła-Karwowska M, Zhylitskaya H, Cybińska J, Lis T, Chmielewski PJ, Stępień M. Angew Chem Int Ed, 2016, 55: 14658-14662

325 Gońka E, Chmielewski PJ, Lis T, Stępień M. J Am Chem Soc, 2014, 136: $16399-16410$

326 Żyła M, Gońka E, Chmielewski PJ, Cybińska J, Stępień M. Chem Sci, 2016, 7: 286-294

327 Yokoi H, Hiraoka Y, Hiroto S, Sakamaki D, Seki S, Shinokubo H. 
Nat Commun, 2015, 6: 8215

328 Ito S, Tokimaru Y, Nozaki K. Angew Chem Int Ed, 2015, 54: 72567260

329 Tokimaru Y, Ito S, Nozaki K. Angew Chem Int Ed, 2018, 57: 98189822

330 Mishra S, Krzeszewski M, Pignedoli CA, Ruffieux P, Fasel R, Gryko DT. Nat Commun, 2018, 9: 1714

331 Ito Y, Christodoulou C, Nardi MV, Koch N, Kläui M, Sachdev H, Müllen K. J Am Chem Soc, 2015, 137: 7678-7685

332 Błoński P, Tuček J, Sofer Z, Mazánek V, Petr M, Pumera M, Otyepka M, Zbořil R. J Am Chem Soc, 2017, 139: 3171-3180

333 Agnoli S, Favaro M. J Mater Chem A, 2016, 4: 5002-5025

334 Tang YB, Yin LC, Yang Y, Bo XH, Cao YL, Wang HE, Zhang WJ, Bello I, Lee ST, Cheng HM, Lee CS. ACS Nano, 2012, 6: 1970-1978

335 Wu ZS, Ren W, Xu L, Li F, Cheng HM. ACS Nano, 2011, 5: 5463 5471

336 Han J, Zhang LL, Lee S, Oh J, Lee KS, Potts JR, Ji J, Zhao X, Ruoff RS, Park S. ACS Nano, 2013, 7: 19-26

337 Lv R, Chen G, Li Q, McCreary A, Botello-Méndez A, Morozov SV, Liang L, Declerck X, Perea-López N, Cullen DA, Feng S, Elías AL, Cruz-Silva R, Fujisawa K, Endo M, Kang F, Charlier JC, Meunier V, Pan M, Harutyunyan AR, Novoselov KS, Terrones M. Proc Natl Acad Sci USA, 2015, 112: 14527-14532

338 Yu X, Han P, Wei Z, Huang L, Gu Z, Peng S, Ma J, Zheng G. Joule, 2018, 2: 1610-1622

339 Jiao Y, Zheng Y, Jaroniec M, Qiao SZ. J Am Chem Soc, 2014, 136 : 4394-4403

340 Dou C, Saito S, Matsuo K, Hisaki I, Yamaguchi S. Angew Chem Int
Ed, 2012, 51: 12206-12210

341 Osumi S, Saito S, Dou C, Matsuo K, Kume K, Yoshikawa H, Awaga K, Yamaguchi S. Chem Sci, 2016, 7: 219-227

342 Cloke RR, Marangoni T, Nguyen GD, Joshi T, Rizzo DJ, Bronner C, Cao T, Louie SG, Crommie MF, Fischer FR. J Am Chem Soc, 2015, 137: 8872-8875

343 Wang S, Zhang L, Xia Z, Roy A, Chang DW, Baek JB, Dai L. Angew Chem Int Ed, 2012, 51: 4209-4212

344 Ci L, Song L, Jin C, Jariwala D, Wu D, Li Y, Srivastava A, Wang ZF, Storr K, Balicas L, Liu F, Ajayan PM. Nat Mater, 2010, 9: 430-435

345 Wang XY, Wang JY, Pei J. Chem Eur J, 2014, 21: 3528-3539

346 Wang XY, Zhuang FD, Wang RB, Wang XC, Cao XY, Wang JY, Pei J. J Am Chem Soc, 2014, 136: 3764-3767

347 Zhong Z, Wang XY, Zhuang FD, Ai N, Wang J, Wang JY, Pei J, Peng J, Cao Y. J Mater Chem A, 2016, 4: 15420-15425

348 Matsui K, Oda S, Yoshiura K, Nakajima K, Yasuda N, Hatakeyama T. J Am Chem Soc, 2018, 140: 1195-1198

349 Nakatsuka S, Yasuda N, Hatakeyama T. J Am Chem Soc, 2018, 140: 13562-13565

350 Kawai S, Nakatsuka S, Hatakeyama T, Pawlak R, Meier T, Tracey J, Meyer E, Foster AS. Sci Adv, 2018, 4: eaar7181

351 Müller M, Behnle S, Maichle-Mössmer C, Bettinger HF. Chem Commun, 2014, 50: 7821-7823

352 Krieg M, Reicherter F, Haiss P, Ströbele M, Eichele K, Treanor MJ, Schaub R, Bettinger HF. Angew Chem Int Ed, 2015, 54: 82848286

353 Dosso J, Tasseroul J, Fasano F, Marinelli D, Biot N, Fermi A, Bonifazi D. Angew Chem Int Ed, 2017, 56: 4483-4487 